Portland State University

PDXScholar

$7-27-2020$

\title{
No Oxygen, No Water: the Unique Physiology of Annual Killifish Embryos Across Development
}

Daniel Erik Zajic

Portland State University

Follow this and additional works at: https://pdxscholar.library.pdx.edu/open_access_etds

Part of the Biology Commons, and the Biophysics Commons

Let us know how access to this document benefits you.

Recommended Citation

Zajic, Daniel Erik, "No Oxygen, No Water: the Unique Physiology of Annual Killifish Embryos Across Development" (2020). Dissertations and Theses. Paper 5508.

https://doi.org/10.15760/etd.7382

This Dissertation is brought to you for free and open access. It has been accepted for inclusion in Dissertations and Theses by an authorized administrator of PDXScholar. Please contact us if we can make this document more accessible: pdxscholar@pdx.edu. 
No Oxygen, No Water: The Unique Physiology

of Annual Killifish Embryos Across Development

by

Daniel Erik Zajic

A dissertation submitted in partial fulfillment of the requirements for the degree of

Doctor of Philosophy

in

Biology

\author{
Dissertation Committee: \\ Jason E. Podrabsky, Chair \\ Bradley Buckley \\ Suzanne Estes \\ Thomas Hancock \\ Gwen Shusterman
}

Portland State University

2020 
(C) 2020 Daniel Erik Zajic 


\begin{abstract}
For most vertebrates, an abundance of oxygen is necessary for the production of ATP and the maintenance of cellular homeostasis. The absence of oxygen, even for brief periods, quickly leads to ATP depletion which can lead to irreparable damages to sensitive organs, such as the brain and heart. However, certain vertebrates demonstrate an extraordinary ability to thrive and recover fully from periods of no oxygen (anoxia). The annual killifish (Austrofundulus limnaeus) lives in ephemeral ponds in the Maracaibo basin of Venezuela and their embryos have the remarkable ability to not only survive anoxic periods for months, but also dehydrating conditions. Survival of this species is dependent on the ability for embryos to enter profound metabolically dormancy termed diapause, as part of their normal life cycle. Survival of dehydrating conditions in A. limnaeus embryos is believed to be achieved through reduction of evaporative water loss and thus is likely to highly limit gas exchange. Thus, embryos exposed to dehydration stress may self-impose severe oxygen stress in order to survive without water. There have been many advances in understanding the extreme anoxia tolerance of this system, but little research has been done on dehydration tolerance and how it relates to anoxia tolerance or stress tolerance in general. There is even less known about the evolutionary route that led to increased stress tolerance associated with diapause in A. limnaeus. I hypothesize that anoxia tolerance is a pre-adaptation that allowed for the evolution of dehydration tolerance. Thus, I predict that $A$. limnaeus embryos exposed to dehydrating conditions will show similar responses at the molecular level to embryos exposed
\end{abstract}


to anoxia. A quintessential feature of anoxia-tolerance is the accumulation of neurotransmitter $\mathrm{y}$-aminobutyric acid (GABA). Despite knowledge of the accumulation of GABA in A. limnaeus, little is known about its role in embryos during anoxia or if it plays a role during dehydration stress. The overall goals of this project were to: (1) determine how GABA accumulation relates to stress tolerance of $A$. limnaeus embryos, and (2) explore the physiological mechanisms that allow A. limnaeus embryos to survive dehydration stress by monitoring survival, measuring oxygen consumption, and performing a metabolomics analysis.

This project was the first to show that inhibition of GABA production or degradation leads to a reduction in anoxia tolerance. Only embryos that can produce GABA are able to successfully recover from long-term anoxia which may be suggestive of its role as a neurotransmitter, an energy source, and/or an antioxidant during anoxia and aerobic recovery. This project uncovered many new aspects of the biology of $A$. limnaeus embryos during dehydration stress. Diapausing embryos are able to survive extremely dehydrating conditions for over 1.5 years, while developing embryos are able to survive for over 100 days. Embryos in diapause respond to dehydration stress by increasing rates of oxygen consumption while post-diapause II embryos either exhibit the same or reduced rates of oxygen consumption when compared to aqueous embryos. Dormant and actively developing embryos respond to dehydration stress in an active manner by significantly altering their metabolic profile. A number of metabolites accumulate during exposure to dehydration stress that may play an 
important role in survival, including the identification of known antioxidants and neuroprotectants. In addition, a number of unique metabolites not yet discussed in the dehydration literature are identified. Despite high oxygen availability, embryos accumulate the anaerobic glycolytic end-product lactate and neurotransmitter GABA. We show that there is undoubtedly overlap in the molecular responses to anoxia and dehydration stress. However, the response to dehydration stress is complex and we have only just begun to scratch the surface into understanding survival of $A$. limnaeus embryos during this crucial time in their life cycle. 


\section{Dedication}

I dedicate this to anyone that has

ever felt disheartened or not good enough

because of a grade they earned in a class

or by a score they received on a standardized test.

I also want to thank all of the humor and memes

that got me through graduate school, of which I will share

one that embodies my experience in writing this dissertation:

Writing is that thing at the eye doctor where they say,

"Which of these is better?"

And you can't actually tell, but you pick one because you have to. But it's for every sentence, always, for the rest of your life. 


\section{Acknowledgments}

The completion of this dissertation would not have been possible without the help and support of so many people. Firstly, I would like to sincerely thank my mentor and advisor Jason Podrabsky for his support and encouragement throughout my project. This dissertation is evidence of what an insightful and caring advisor he is. Jason has always been a positive and reassuring figure for me in and outside of the lab. I thank the members of my committee: Bradley Buckley, Suzanne Estes, Thomas Hancock, and Gwen Shusterman. Their feedback and encouragement in all aspects of research and life has been invaluable. Brad always had words of encouragement and the breaks from science to talk about the Blazers were much appreciated. Suzanne was always very supportive and introduced me to so many amazing undergraduate students that helped me with my work and become a better mentor. Tom showed me what true passion for teaching can lead to and I am forever grateful for everything I have learned from him about physiology. I would like to thank everyone in the biology department at Portland State - students, staff, and faculty - for their support in shaping me into the person I am today.

Much of what molded me into the individual and researcher I am today is attributed to my former labmates, who created a lab environment full of comradery, shenanigans, and baked goods: Amie Romney, Claire Riggs, Cody Steven Woll, and Josiah Wagner. I especially owe a lot to Claire Riggs for her guidance - many days I felt like a lost puppy trying to find his way and she was always there to help. In addition, there have been so many people that have 
contributed directly or indirectly to this dissertation and my success in graduate school: Andrew Ashford, Kenia Chavez, Meranda Corona, Erin Davis, Decatur Foster, lan Garrett, Lindsay Holden, Kate Kouba, Rosey Le, Emily Morse, Jonathon Nicholson, Rachel Palmer, Malcolm Peavy, Adalid Pelayo, Jake Roush, Lauren Rust, Amy Seufert, Motu Sipelii, Brie Tripp, Rachel Wilson, and Christina Yildirimer. I am especially thankful to Rachel Wilson for being the best friend and colleague I could ask for. The entirety of this research would not have been possible without the help of all of the amazing undergraduate work-study students who helped in our fishroom over the years.

I also want to thank my non-graduate school friends whose support and encouragement kept me going. I thank all of my family for bearing with me over the last five years and even if they did not exactly know what I was doing, they were supportive and understanding. Finally, thank you to my partner Tom for being so loving and caring over the years, especially during the crazy COVID-19dissertation-writing phase. 


\section{Table of Contents}

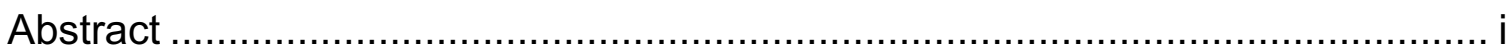

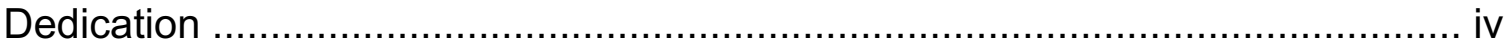

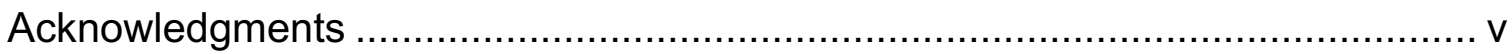

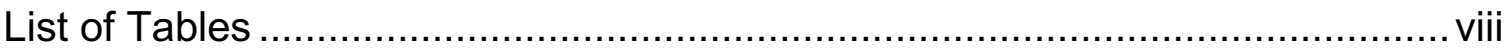

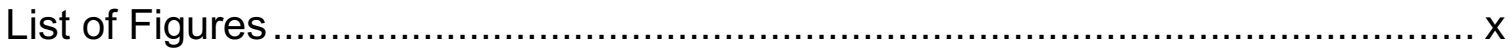

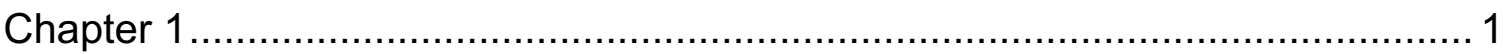

Two fish with one stone?

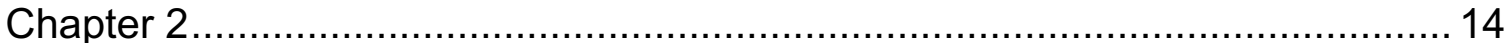

GABA daba doo, anoxia got nothing on you: GABA metabolism is crucial for long-term survival in annual killifish embryos

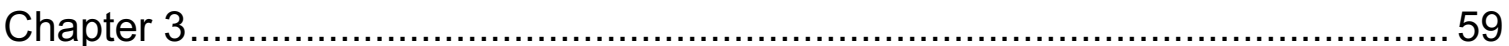

No water, no problem: Stage-specific metabolic responses to dehydration stress

in annual killifish embryos

Chapter 4

Metabolomics analysis provides insight on survival of annual killifish

(Austrofundulus limnaeus) embryos during dehydration stress

Chapter 5

GABA daba done: Summary and future directions

References

Appendix: Supplemental File Information 


\section{List of Tables}

Table 2.1.

Segmental linear regression analysis of GABA and lactate accumulation during exposure to anoxia

Table 3.1. 79

Oxygen consumption $\left(\mathrm{VO}_{2}\right)$ of Austrofundulus limnaeus embryos incubated in aerial or aqueous conditions

Table 4.1. 116

Total number of significant metabolites (among 673 total metabolites identified) that were significantly affected by short and long-term aerial exposure in DII and post-DIl embryos initially exposed at WS 36

Table 4.2.

Top 40 significantly upregulated metabolites during short-term aerial exposure in DIl embryos normalized to DNA concentration.

Table 4.3. 118

Top 40 significantly upregulated metabolites during long-term aerial exposure in DIl embryos normalized to DNA concentration.

Table 4.4. 119

Top 40 significantly upregulated metabolites during short-term aerial exposure in post-DIl embryos normalized to DNA concentration.

Table 4.5. 120

Top 40 significantly upregulated metabolites during long-term aerial exposure in post-DIl embryos normalized to DNA concentration.

Table 4.6.

Top 30 significantly downregulated metabolites during short-term aerial exposure in DIl embryos normalized to DNA concentration.

Table 4.7.

Top 34 significantly downregulated metabolites during long-term aerial exposure in DIl embryos normalized to DNA concentration.

Table 4.8. 123

Top 40 significantly downregulated metabolites during short-term aerial exposure in post-DIl embryos normalized to DNA concentration. 
Table 4.9.

Top 40 significantly downregulated metabolites during long-term aerial exposure in post-DIl embryos normalized to DNA concentration.

Table 4.10.

125

Metabolic pathways that were significantly enriched by short and long-term aerial exposure in DII and post-DII embryos normalized to DNA concentration 


\section{List of Figures}

Figure 1.1. The life cycle of Austrofundulus limnaeus ................................... 12

Figure 1.2. The anoxia tolerance of various vertebrates across development .... 13

Figure 2.1. GABA and lactate accumulation in embryos of Austrofundulus limnaeus during exposure to anoxia and aerobic recovery from anoxia at $25^{\circ} \mathrm{C}$

Figure 2.2. The relationship between lethal time to $50 \%$ mortality $\left(\mathrm{LT}_{50}\right)$ and anoxic GABA and lactate accumulation in embryos of $A$. limnaeus

Figure 2.3. Survival of embryos to $800 \mu \mathrm{mol} \mathrm{I}^{-1}$ ethyl ketopentenoate (EKP) in normoxia and anoxia

Figure 2.4. GABA and lactate accumulation during inhibition of the GABA synthesis and degradation pathway in Wourms' stage 36 embryos exposed to anoxia

Figure 2.5. Survival of Wourms' stage 36 embryos continuously exposed to GAD inhibitor EKP, SSADH inhibitor DEAB, and GABA-T inhibitor vigabatrin in anoxia

Figure 2.6. GABA accumulation during inhibition of diamine oxidase (DAO) by amiloride in Wourms' stage 36 embryos exposed to anoxia

Figure 2.7. Glutamate decarboxylase (GAD) activity in adult male $A$. limnaeus brain homogenates in normoxia at $25^{\circ} \mathrm{C}$

Figure 2.8. Glutamate decarboxylase (GAD) activity in A. limnaeus embryo homogenates across development in normoxia at $25^{\circ} \mathrm{C}$.

Figure 2.9. Transcript levels for GABA, polyamine, lactate, and ethanol pathways in $A$. limnaeus as determined by RNA sequencing 56

Figure 2.10. Transcript levels for GABA signaling and cotransporters in $A$. limnaeus as determined by RNA sequencing

Figure 2.11. The relationship between female age and rates of GABA and lactate accumulation in anoxic Wourms' stage 36 embryos . 58

Figure 3.1. Survival of Austrofundulus limnaeus embryos incubated in aerial conditions 
Figure 3.2. Oxygen consumption of Austrofundulus limnaeus embryos in aerial and aqueous conditions

Figure 3.3. Rate of oxygen consumption in Austrofundulus limnaeus embryos exposed to aqueous and aerial conditions

Figure 4.1. Schematic of the experimental design and sampling regimen used for metabolomics analysis

Figure 4.2. The relationship of aerial exposure on mass and protein concentration in DII and post-DII embryos....

Figure 4.3. Heat maps of fold change from $t=0$ of 673 metabolites detected in embryos.

Figure 4.4. Metabolites significantly down or upregulated during long-term aerial exposure in DII and post-DII embryos

Figure 4.5. Indications of altered transsulfuration activity and antioxidant utilization in DII and post-DII embryos.

Figure 4.6. Changes in gamma-glutamyl amino acid metabolism in DII and post-

DIl embryos

Figure 4.7. Changes in creatine metabolism in DII and post-DII embryos

Figure 4.8. Changes in glycolysis and pentose phosphate pathway metabolism in DII and post-DIl embryos

Figure 4.9. Changes in nicotinate and nicotinamide metabolism in DII and postDIl embryos 


\section{Chapter 1}

\section{Two fish with one stone?}

Model organisms are extensively used in research as they are accessible and convenient systems to study a particular area or question in biology. Traditionally, only a select number of organisms have been widely studied, but with so many advancements in modern technology and research tools, researchers are now able to extend beyond the status quo and explore understudied and unusual organisms. A recent paper by Goldstein and King (2016) argues that some of the biggest future discoveries in biology could come from the development and study of new and atypical model organisms. The origin of model organisms was centralized around wanting to study a particular subset of biology; thus investigating a simple, controllable organism that enables experimental work was logical. Without contributions from studying bacteria, corn, and yeast, much of what we know about basic molecular biology would not exist. A lot of our understanding of developmental biology stemmed from researching flies, worms, Arabidopsis, and mice. These systems were mainly chosen as they were smaller, simpler, and faster growing than more complex systems (e.g., humans) (Russell et al., 2017). Goldstein and King (2016) emphasize the need for researchers to ask questions that they know their model organism is appropriate to answer, which was paramount to the development of this dissertation. 
For most animals, an ample supply of oxygen and water is essential for survival. Under aerobic conditions, most of the energy (i.e., ATP) used by a cell is captured through oxidative phosphorylation. This process relies on oxygen as the final electron acceptor in an electron transport chain that drives synthesis of ATP through generation of a proton motive force across the inner mitochondrial membrane. Thus, without adequate oxygen, this pathway cannot produce ATP needed for cell survival. Animals respond to a lack of oxygen by shifting energy production to anaerobic mechanisms, typically anaerobic glycolysis. However, glycolysis alone often cannot meet the energetic demands of an organism. A seemingly simple solution to accommodate the low rates of ATP production is to reduce the ATP required by the organism. However, the ability to suppress metabolism and substantially reduce ATP turnover, and thus survive periods of anoxia (no oxygen), in vertebrates is rare. In most organisms, even brief episodes of low oxygen supply can cause irreparable damages to vital organs, such as the brain and heart (Larson et al., 2014). Few vertebrates are able to survive long bouts of no oxygen (anoxia), while even fewer are able to thrive and recover fully from these anoxic periods. In addition, water is essential for life on Earth. Water allows for transportation of materials, function of molecular machinery, and facilitation of chemical reactions. Further, water is important to the structure, stability, dynamics, and function of biological macromolecules. Aquatic organisms have evolved to depend on nearly constant immersion in water to support basic physiological functions. However, in its absence, some aquatic organisms have been able to adapt and survive. 
The annual killifish, Austrofundulus limnaeus, survives in ephemeral ponds in the coastal deserts of Venezuela (Podrabsky and Hand, 1999). Like most annual killifishes, they have a unique life history that has allowed for the evolution of unique physiological mechanisms in their embryos (See Fig 1.1 for visual representation of $A$. limnaeus life cycle) (Podrabsky et al., 2016). Adult fish are abundant during the rainy seasons, constantly spawning and producing eggs which they deposit into the muddy substrate. While there is an abundance of water, embryos are deposited in the sediment where high microbial activity creates a severely hypoxic or anoxic environment (Podrabsky et al., 1998). Thus, embryos face periods of little to no oxygen availability as part of their normal development. As the ponds dry up and the dry season begins, adult fish die and the embryos must endure the entirety of the dry season until water returns and they can hatch. Embryos now must face dehydrating conditions, the potential for exposure to UV light, extreme temperatures, and salinity stress (Podrabsky et al., 2016). Survival is attributed to the ability of embryos of $A$. limnaeus to enter drastic metabolic dormancy (diapause) as a part of their normal development (Podrabsky and Hand, 1999). Annual killifish embryos can enter diapause at three distinct stages termed diapause I, II, and III. Diapause I (DI) occurs prior to embryonic axis formation at 4 days post fertilization (dpf), diapause II (DII) occurs approximately midway through embryonic development, and diapause III (DIII) occurs at the end of embryonic development prior to attaching (Podrabsky et al., 2017; Wourms, 1972b; Wourms, 1972c). Their anoxia tolerance peaks at DII and is maintained for a few days after breaking diapause II, before decreasing 
thereafter (Podrabsky et al., 2007; Podrabsky et al., 2012b; Riggs and Podrabsky, 2017). Thus, A. limnaeus provides a gradient of anoxia-tolerant and anoxia-sensitive stages, which allows for a comparative study within a single system.

Respiration during dormancy and rates of water loss are closely related: if oxygen can pass in and carbon dioxide can pass out of the organism, so can water vapor (Danks, 2000). Survival of dehydrating conditions in A. limnaeus embryos is achieved through reduction of evaporative water loss and thus is likely to highly limit gas exchange (Podrabsky et al., 2001). Diapausing insects have been seen to prevent water loss by having very low cuticle permeability, which is attributed to the formation of a waxy later on their cuticle full of longchain hydrocarbons (Chung and Carroll, 2015; Danks, 2000). To prevent water loss but maintain an adequate amount of oxygen intake and carbon dioxide release, many insects exhibit cyclic respiration. To survive dehydrating conditions, $A$. limnaeus embryos may self-impose severe hypoxia and anoxia in order to maintain their embryonic water content. Despite recent progress on mechanisms of anoxia tolerance in this system, much remains unknown about desiccation tolerance and its relationship with anoxia tolerance or stress tolerance in general. Even less is known about the evolutionary route that led to increased stress tolerance associated with diapause in A. limnaeus. I propose that anoxia tolerance is a pre-adaptation that allowed the evolution of dehydration tolerance. Thus, I predict that $A$. limnaeus embryos exposed to dehydrating conditions will show similar responses at the molecular level to 
embryos exposed to anoxia. To better understand the potential interplay between the two stresses, we must first look at each stressor individually.

\section{Anoxia}

The embryos of the annual killifish $A$. limnaeus exhibit a profound tolerance of anoxia and, when accounting for temperature, they are the most anoxia-tolerant vertebrate known to exist (Fig 1.2) (Podrabsky et al., 2012b). Unlike most developing embryos, $A$. limnaeus displays an increase in anoxia tolerance during development, which peaks at DII (24-32 dpf), is maintained for several days after embryos exit from DII, and then decreases as embryos develop towards hatching. Stress tolerance of $A$. limnaeus embryos in general seems to parallel with their ability to survive anoxia (Machado and Podrabsky, 2007; Podrabsky et al., 2007; Riggs and Podrabsky, 2017; Wagner et al., 2019). The ability to study intraspecific variation in stress tolerance within a single system makes $A$. limnaeus the ideal vertebrate system for comparative stress tolerance studies. The most anoxia tolerant stages (DII and Wourms' stage [WS] 36) are composed mostly of cardiac tissue (functional heart) and neural tissue, which may provide insight into how these tissues, which are the most anoxiasensitive in other organisms, are able to survive seemingly undamaged after long exposures to anoxia. In addition to comparing anoxia-tolerant and anoxiasensitive stages within A. limnaeus, comparison between the anoxia-tolerant DII and WS 36 stages may shed light on unique mechanisms of survival. The former stage is already metabolically depressed and in arrested development, whereas 
the latter is actively developing and must quickly and dramatically reduce their metabolism in response to their environment (enter quiescence) (Podrabsky et al., 2007), thus these embryos may implement separate mechanisms to survive stressful conditions.

Survival during anoxia is attributed to an organism's ability to greatly reduce their metabolic rate. Maintaining adequate ATP production while limiting ATP consumption is paramount for survival in the presence of no oxygen (Hand and Podrabsky, 2000). Metabolic depression is achieved through physiological and biochemical alterations, mainly reductions in protein synthesis, ion regulation, and macromolecule biosynthesis (Fraser et al., 2002; Podrabsky and Hand, 2000; Podrabsky et al., 2007). Protein synthesis accounts for up to $40 \%$ of basal metabolism in mammals and up to $90 \%$ of total metabolism in fish (Buttgereit and Brand, 1995; Fuery et al., 1998; Podrabsky and Hand, 2000; Smith and Houlihan, 1995). The ability to survive in anoxia is reliant on the embryo's ability to undergo severe metabolic depression, which is largely facilitated by severely depressing protein synthesis. Diapause II embryos maintain high $[A T P] /[A D P]$ ratios and adenylate energy charge during diapause, which supports a coordinated downregulation of ATP production and consumption. This is likely a key to the ability of DII embryos to survive long bouts without oxygen and thus overcome the stress of anoxia. However, when exposed to anoxia, both diapausing and developing embryos experience a drop in ATP levels (by as much as $80 \%$ ) which is accompanied by an associated drop in heat dissipation (a proxy for metabolism) (Podrabsky et al., 2012a). Thus, 
even dormant embryos that are "prepared" for stress must react to anoxia at the metabolic level in order to survive.

In response to anoxia, many anoxia-tolerant organisms produce the neurotransmitter y-aminobutyric acid (GABA) (Hylland and Nilsson, 1999; Nilsson and Lutz, 1991). GABA has been found to provide excitatory actions in the developing vertebrate nervous system, but conversely, typically functions as an inhibitory neurotransmitter in adults (Ben-Ari, 2002). It has been suggested that GABA (in micromolar concentrations) can act as a mediator of metabolic depression in the anoxic crucian carp brain (Nilsson, 1992). In the anoxiasensitive mammalian brain, a rapid fall in ATP levels in response to no oxygen leads to general depolarization and release of excitatory amino acids (e.g., glutamate), ultimately concluding in neuronal cell death (Nilsson and Renshaw, 2004). A main factor that differentiates anoxia-tolerant and anoxia-sensitive species is their ability to maintain glutamate homeostasis in response to low oxygen levels. The ability to convert an excitatory neurotransmitter (glutamate) to an inhibitor neurotransmitter (GABA) may be beneficial to organisms trying to depress their metabolism. Anoxia-tolerant vertebrates are able to decrease their metabolic rate by $70 \%$ or more during anoxia, while at the same time increasing GABA levels (Nilsson, 1992). Increased GABA levels are thought to protect the brain of many organisms from excitotoxic cell death during hypoxic and anoxic conditions (Podrabsky et al., 2007). However, embryos of A. limnaeus accumulate millimolar quantities of GABA during prolonged bouts of anoxia, an amount that is over 1000 times higher than in other anoxia tolerant species 
(Podrabsky et al., 2007). It is likely that high glutamate decarboxylase activity can explain the substantial amount of GABA found in embryos exposed to anoxia. The high levels of GABA produced in embryos of $A$. limnaeus suggest that it is likely to act as an inhibitory neurotransmitter at this stage of development and perhaps may serve a purpose other than neurotransmission when embryos are under stress.

\section{Dehydration}

Water is essential for life and the threat of dehydration stress puts organisms at risk. However, some organisms have adapted mechanisms for survival when faced with water stress. Many anhydrobiotic organisms accumulate polyhydroxy compounds such as trehalose to essentially "replace" water in order to maintain the hydrogen bonds necessary for structural integrity of biological structures (Crowe and Madin, 1974; Erkut et al., 2011; Wright, 1989). This mechanism allows for the organisms to survive "life without water" and is exhibited in a number of species, including tardigrades (Hypsibius dujardini), brine shrimp cysts (Artemia franciscana), and the dauer larva stage of Caenorhabditis elegans (Crowe et al., 1984; Erkut and Kurzchalia, 2015; Erkut et al., 2011). Alternatively, organisms can reduce water loss behaviorally by burrowing as seen in the African lungfish (Protopterus aethiopicus) or by producing a cocoon as seen in some amphibian species (Fishman et al., 1992; Toledo and Jared, 1993). 
Embryos of $A$. limnaeus experience unique resistance to desiccation unseen by aquatic vertebrates (Podrabsky et al., 2001). However, dehydration tolerance of these embryos has received much less attention than other aspects of their biology, and many questions remain regarding how long embryos can survive without water and the molecular mechanisms that support survival. Embryos exposed to dehydration stress lose water in the first few days, but by day seven, the rate of water loss is near zero. Importantly, most of this water is lost from the extraembryonic perivitelline compartment, while the embryo appears to remain fully hydrated (Podrabsky et al., 2001). The lack of further water loss suggests embryos may be dramatically reducing gas exchange with their environment to reduce evaporative water loss. As a consequence, reduced permeability to water vapor may lead to highly limited gas exchange in general and thus self-imposed hypoxia or anoxia. This oxygen deprivation may initiate embryonic quiescence (halting development), which is seen in other postdiapause embryos in response to anoxia (Podrabsky et al., 2012b).

Organisms living in aquatic habitats are especially susceptible to low oxygen availability. This is attributed to the low oxygen capacity of water and factors such as salinity, temperature and turbidity affecting oxygen levels. The levels of photosynthesis during the day and night create oxygen fluctuations for aquatic organisms; photosynthetic levels are lower during periods of low light, such as during the night and the winter season, which can create daily hypoxic conditions for aquatic organisms. Thus, hypoxia and anoxia are common elements of many aquatic habits, and many aquatic vertebrates face acute or 
chronic periods of low oxygen. I hypothesize that anoxia tolerance and dehydration tolerance are linked, thus embryos are using the same mechanisms to survive the stress of anoxia as they are to survive desiccation. Thus, I predict that embryonic stages that have evolved mechanisms to survive low oxygen will have the molecular foundation to survive without water.

\section{Organization of remaining chapters}

This work is an exploration of the physiology of $A$. limnaeus embryos in response to two unique stresses: anoxia and dehydration. In Chapter 2, entitled GABA daba doo, anoxia got nothing on you: GABA metabolism is crucial for long-term survival in annual killifish embryos, I measure GABA and lactate in A. limnaeus embryos across development and during exposure to long-term anoxia and aerobic recovery from anoxia. I use pharmacological inhibitors and transcript data to suggest roles for GABA and lactate during anoxia and recovery. In

Chapter 3, entitled No water, no problem: Stage-specific metabolic responses to dehydration stress in annual killifish embryos, I assess the dehydration tolerance of embryos across development and then look at rates of oxygen consumption in dormant and actively developing embryos during aerial incubation. I interpret the data as they relate to the ecology of embryonic development in annual killifishes. In Chapter 4, entitled Metabolomics analysis provides insight on survival of annual killifish (Austrofundulus limnaeus) embryos during dehydration stress, I perform a metabolomics analysis on embryos exposed to dehydrating conditions to identify potential key metabolites and metabolic pathways that assist in 
survival of $A$. limnaeus embryos during dehydration stress. In the final chapter, Chapter 5, entitled GABA daba done: Summary and future directions, I review the findings from Chapters $2-4$, integrate what I have found as it relates to $A$. limnaeus and organismal stress tolerance, and suggest future directions for research.

\section{Significance}

An organism may have a single response to multiple stressors, sharing signaling pathways (cross-talk) or protective mechanisms (cross-tolerance) (Sinclair et al., 2013). Cross-talk occurs when shared regulatory pathways trigger separate mechanisms of protection against different stresses. Conversely, some forms of cellular protection can be effective against multiple forms of stress. A mechanism that can protect against multiple forms of stress is known as crosstolerance. Understanding the relationship between anoxia and dehydration and the response these stressors induce in A. limnaeus will expand the current knowledge of co-evolution and cross-tolerance. I study a system that experiences two major stresses as part of their normal development, which undoubtedly has led to unique physiological adaptations. Together, these chapters aim to unravel the molecular underpinnings behind the physiology of $A$. limnaeus embryos in response to anoxia and dehydration, which will provide insight into how organisms combat different stressors and help explain why $A$. limnaeus thrive where other organisms fail. 


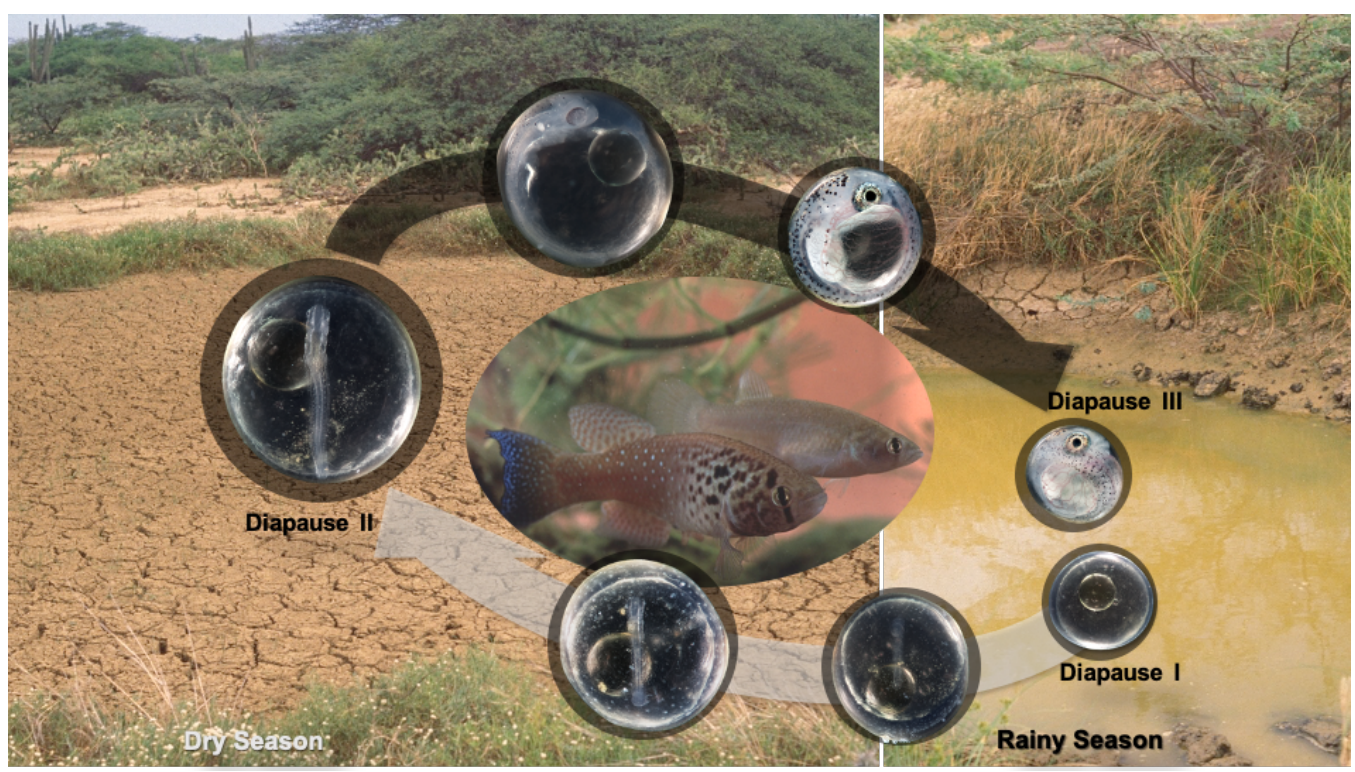

Figure 1.1. The life cycle of Austrofundulus limnaeus. Adult fish spawn during the rainy season and produce embryos that are able to enter metabolic dormancy (diapause) in three distinct stages as part of normal development. Embryos survive the entirety of the dry season by entrance into diapause II. When the ponds return with the rainy season, embryos continue developing towards hatching. 


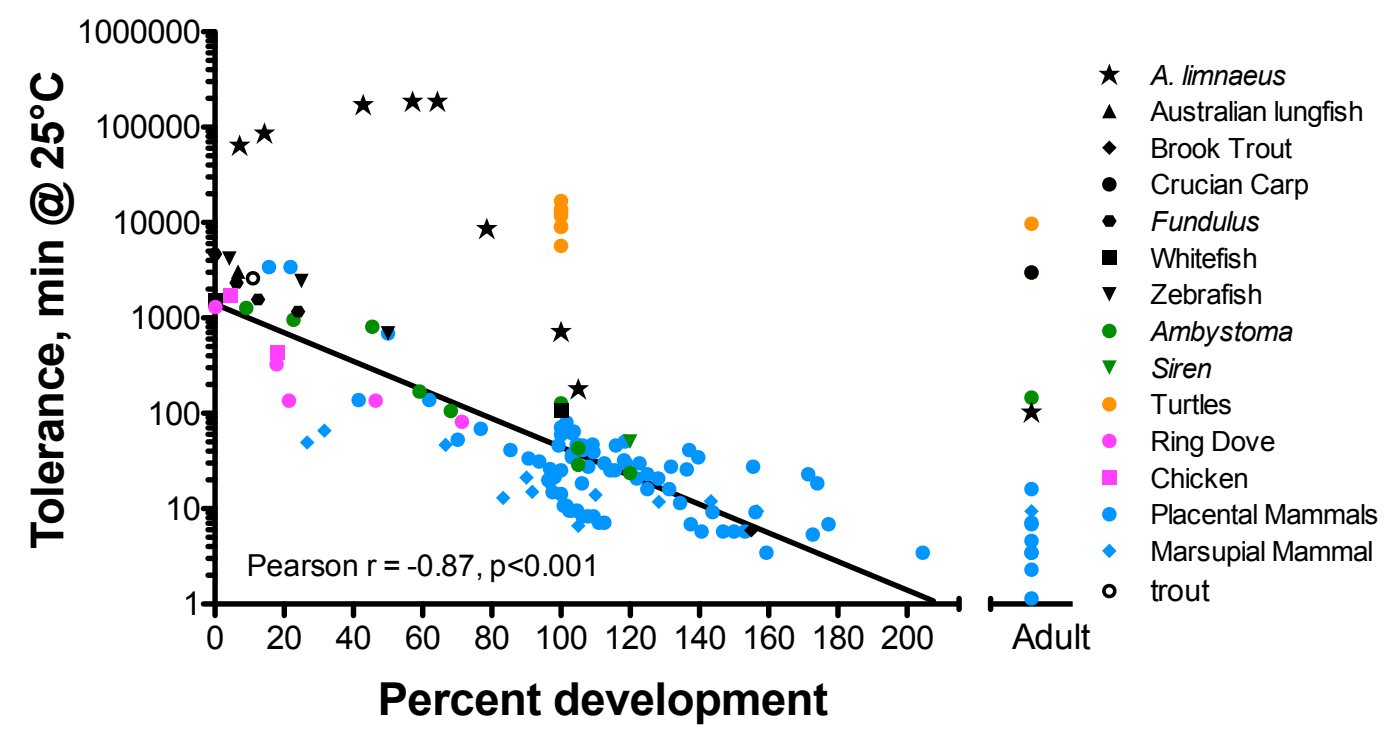

Figure 1.2. The anoxia tolerance of various vertebrates across development. The embryos of Austrofundulus limnaeus (star symbols) exhibit an anoxia tolerance two orders of magnitude higher than any other vertebrate in addition to an initial rise in anoxia tolerance during development. All data has been normalized to $25^{\circ} \mathrm{C}$ assuming a $Q_{10}$ of 2 . The regression analysis performed was calculated without the embryos of $A$. limnaeus and turtle hatchlings, as they were outliers. This figure is from Podrabsky et al. (2012b) and additional details are outlined there. 


\section{Chapter 2}

\section{GABA daba doo, anoxia got nothing on you: GABA metabolism is crucial for long-term survival in annual killifish embryos}

This chapter has previously been submitted for publication:

Zajic D, and Podrabsky J. GABA daba doo, anoxia got nothing on you: GABA metabolism is crucial for long-term survival in annual killifish embryos. $J$ Exp Biol Submitted: 2020.

\section{Introduction}

In most organisms, even brief episodes of low oxygen can cause irreparable damages to vital organs, such as the brain and heart (Larson et al., 2014). Few vertebrates are able to survive long bouts of anoxia, while even fewer are able to thrive and recover from anoxic stress. A small number of vertebrates have evolved the ability to tolerate long-term anoxia, and these organisms share a number of physiological, cellular, and biochemical characteristics that appear to be of fundamental importance for survival without oxygen (Bundgaard et al., 2020; Podrabsky et al., 2007; Riggs et al., 2018; Thompson et al., 2013). One common feature found in all groups of anoxia tolerant vertebrates is the production of GABA, an inhibitory neurotransmitter, in response to anoxia (Hylland and Nilsson, 1999; Nilsson and Lutz, 1991). GABA production in the brain during anoxia is thought to protect against excitotoxic cell death and, generally, relatively small concentrations of GABA (micromolar) are required to 
achieve neuroprotection (Gilby et al., 2005; Johns et al., 2000; Lutz and Milton, 2004; Pamenter et al., 2011). Embryos of the annual killifish, Austrofundulus limnaeus, can survive for months without oxygen, and long-term survival of anoxia is associated with accumulation of millimolar quantities of GABA (Podrabsky et al., 2007). The accumulation of high concentrations of GABA in anoxic embryos of $A$. limnaeus suggests a role beyond neurotransmission. In this paper we explore the potential role of GABA production in supporting long-term survival of anoxia in A. limnaeus embryos.

Austrofundulus limnaeus survives in ephemeral ponds in the coastal deserts of Venezuela by producing embryos that arrest development in diapause as a part of their normal development (Podrabsky and Hand, 1999). Embryos can enter diapause at up to three unique developmental stages, termed diapause I, II, and III, which together extend the time for embryonic development to span the duration of the dry season (Podrabsky et al., 2017; Wourms, 1972b; Wourms, 1972c). Unlike most developing embryos, A. limnaeus displays an increase in anoxia tolerance during development, which peaks at diapause II (DII; 24-32 days post fertilization), is maintained for several days after embryos exit from DII, and then decreases as embryos develop towards hatching (Meller et al., 2012; Podrabsky et al., 2007; Riggs and Podrabsky, 2017). At their peak anoxia tolerance, embryos have the remarkable ability to tolerate anoxic conditions for months at $25^{\circ} \mathrm{C}-$ two orders of magnitude higher than any other vertebrate (Podrabsky et al., 1998; Podrabsky et al., 2012b). What is astounding is that the most anoxia tolerant stages, DII and 4 days post-diapause II (dpd; Wourms' 
stage [WS] 36), are composed mostly of characteristically anoxia-sensitive cardiac tissue (functional heart) and neural tissue (Podrabsky and Hand, 1999; Podrabsky et al., 2007). Survival in anoxia is attributed to the ability of embryos to substantially decrease metabolism, either by already being metabolically dormant (in diapause), or through rapidly decreasing metabolism by entering anoxia-induced quiescence (Podrabsky et al., 2012a). Thus, A. limnaeus provides a gradient of anoxia-tolerant and anoxia-sensitive stages, which allows for a comparative study within a single system.

Without oxygen, organisms must shift to anaerobic pathways as a means of supporting catabolic metabolism. Most vertebrates rely on anaerobic glycolysis and the production of lactate to fuel metabolism in the absence of oxygen. In most organisms facing anoxia, a lack of adequate glycolytic ATP production to meet metabolic demand leads to a bioenergetic and redox imbalance and the activation of cell-death pathways (Boutilier, 2001; Jacobson and Raff, 1995). Even if metabolic supply and demand are balanced, survival times in anoxia are typically limited by the negative effects of lactic acidosis, which can be partially offset through increased buffering capacity (Jackson, 1997; Jackson et al., 2000). The ability for $A$. limnaeus embryos to maintain high [ATP]/[ADP] ratios during diapause supports a coordinated downregulation of ATP production and consumption, and is likely a key to the embryos ability to survive long bouts without oxygen during diapause. However, ATP levels are not maintained in developing embryos exposed to anoxia (Podrabsky et al., 2012a), and yet these embryos exhibit similar survival of anoxia (Podrabsky et al., 2007). Thus, in $A$. 
limnaeus there may be multiple mechanisms for achieving extreme anoxia tolerance. Recent progress has been made on understanding multiple factors that may be contributing to the survival of $A$. limnaeus in anoxia (depressed protein synthesis, inhibition of apoptosis, antioxidant capacity, and small noncoding RNAs) (Meller and Podrabsky, 2013; Podrabsky and Hand, 2000; Riggs and Podrabsky, 2017; Wagner et al., 2019).

When exposed to anoxia, embryos of $A$. limnaeus respond by producing large amounts (> $10 \mathrm{mmol} \mathrm{I}^{-1}$ ) of GABA (Podrabsky et al., 2007). GABA is the primary inhibitory neurotransmitter in the adult central nervous system; however, it appears to function in an excitatory manner during early vertebrate development (Ben-Ari, 2002). The high levels of GABA produced in anoxic embryos of $A$. limnaeus suggest a role beyond inhibitory neurotransmission. Despite the massive accumulation of GABA in A. limnaeus embryos, the metabolic origins and function of GABA in A. limnaeus embryos remain unexplored.

Here we report on the long-term metabolic responses of dormant (DII) and actively developing post-diapause II embryos to anoxia and aerobic recovery to evaluate the role of GABA. We evaluate these data in relation to anoxia tolerance by utilizing developmental stages that provide a gradient of anoxia tolerance. We inhibit enzymes responsible for GABA production and degradation to see how GABA and lactate levels are affected and how that affects survival in anoxia. These data support a role for GABA as a metabolic intermediate, and not a metabolic end-product. Here we suggest multiple roles for GABA during anoxia 
and aerobic recovery in A. limnaeus embryos: as a neurotransmitter, as a source of energy, and as an antioxidant. We support our findings with transcript expression data during normal $A$. limnaeus embryonic development and in response to anoxia and aerobic recovery. We explore how long-term survival in anoxia and subsequent aerobic recovery may be supported by accumulation of GABA. Understanding how the most anoxia tolerant stages, composed mostly of cardiac tissue and neural tissue, utilize GABA may provide insight into how these tissues are able to survive long-term anoxia.

\section{Materials and Methods}

\section{Husbandry of A. limnaeus and embryo collection}

Adult annual killifish (Austrofundulus limnaeus) were reared in the Portland State University (PSU) aquatic vertebrate facility and cared for according to established husbandry methods in accordance with approved PSU Institutional Animal Care and Use Committee protocols (PSU IACUC protocols \#33 and 64). Adult $A$. limnaeus were kept in spawning pairs and housed in 9.5 I tanks and collectively 21 tanks were connected to a communal sump and filtration system. Paired adults were allowed to spawn semiweekly and embryos were collected. Embryos were stored at $25^{\circ} \mathrm{C}$ under dark conditions in $15 \times 100$ $\mathrm{mm}$ plastic Petri dishes in media that resembles the environmental conditions from which adults were collected in $1995\left(10 \mathrm{mmol} \mathrm{I}^{-1} \mathrm{NaCl}, 2.15 \mathrm{mmol} \mathrm{I}^{-1} \mathrm{MgCl}_{2}\right.$, $0.8 \mathrm{mmol} \mathrm{l}^{-1} \mathrm{CaCl}_{2}, 0.14 \mathrm{mmol} \mathrm{I}^{-1} \mathrm{KCl}, 1.3 \mathrm{mmol} \mathrm{I}^{-1} \mathrm{MgSO}_{4}$ ) (Podrabsky, 1999; Podrabsky et al., 1998). Embryo medium contained methylene blue $(0.0001 \%)$ 
for the first 4 days post-fertilization (dpf) to prevent fungal growth. To prevent microbial growth and infection, embryos were treated at $4 \mathrm{dpf}$ with two $5 \mathrm{~min}$ rinses of embryo medium containing sodium hypochlorite (Clorox, $0.03 \%$ ) separated by a 5 min rest in embryo medium without sodium hypochlorite. After the last sodium hypochlorite wash, embryos were rinsed with embryo medium containing sodium thiosulfate $(0.005 \%)$ to neutralize remaining sodium hypochlorite. Embryos were then placed in medium containing $10 \mathrm{mg} \mathrm{l}^{-1}$ gentamicin sulfate and allowed to develop at $25^{\circ} \mathrm{C}$ without light to promote entry into DII and prevent breaking of DII until experiments were conducted (28-66 d) (Podrabsky et al., 2010a; Romney et al., 2018). Embryos enter DII at around 24 dpf at $25^{\circ} \mathrm{C}$.

\section{Developmental staging and sampling of embryos}

Diapause II was broken by subjecting embryos to a temperature of $30^{\circ} \mathrm{C}$ and full spectrum light for $48 \mathrm{~h}$ (Meller et al., 2012). Upon breaking DII, embryos were sorted by developmental stage according to Wourms' stage (WS) in concert with recent adaptations by Podrabsky et al. (Podrabsky et al., 2017; Wourms, 1972b; Wourms, 1972c). Experiments were performed on four stages of embryos to capture a gradient of anoxia tolerance levels and physiology: DII (Diapause II, WS 32/33), WS 36 (4 days post-diapause II (dpd)), WS 40 (12 dpd), and WS 42 (20 dpd). DIl embryos are metabolically dormant and have arrested development and thus are primed for anoxia; whereas, post-DIl embryos are metabolically and developmentally active and enter quiescence in response to anoxia (Podrabsky 
et al., 2007). Lethal time to $50 \%$ mortality $\left(\mathrm{LT}_{50}\right)$ in response to anoxia has previously been described (Meller et al., 2012; Podrabsky et al., 2007; Riggs and Podrabsky, 2017). Embryos develop anoxia tolerance as they enter DII $\left(\mathrm{LT}_{50}=\right.$ 65 d) and maintain extreme tolerance for 4-6 days after breaking DII, after which anoxia tolerance decreases dramatically in later stage embryos: WS $36\left(\mathrm{LT}_{50}=\right.$ $74 \mathrm{~d})$, WS $40\left(\mathrm{LT}_{50}=7 \mathrm{~d}\right)$, WS $42\left(\mathrm{LT}_{50}=0.67 \mathrm{~d}\right)$. Embryos were sampled in their respective environmental conditions (anoxia or normoxia) by placing them on a mesh screen and wicking away excess liquid using dry paper towels prior to being placed in a pre-weighed microcentrifuge tube. Samples were then quickly weighed and stored at $-80^{\circ} \mathrm{C}$ until metabolite extraction. Typically, embryos were used from a single spawning event to represent a single replicate; however, in some instances multiple spawning events were combined in order to have sufficient embryos at a specific developmental stage $(N=3-6)$. Sampling of embryos covered the lifespan of $A$. limnaeus and multiple generations, however, control embryos were always sampled to account for any batch or generational effects.

\section{Long-term anoxia exposure, recovery, and sampling}

Embryos were exposed to various periods of anoxia based on their anoxia tolerance, such that the longest period of anoxia matched their $L T_{50}($ Fig 2.1A). Embryos were allowed to recover following long-term anoxia and samples were collected at several time points following aerobic recovery. WS 42 embryos either hatched or were not able to recover following reoxygenation and data for 
recovery were not collected. Embryos were exposed to anoxia in a Bactron III anaerobic chamber (Sheldon Manufacturing, Cornelius, OR, USA), which maintained anoxic conditions by using hydrogen gas and a palladium catalyst to react with any trace oxygen. Anoxic conditions were maintained at $5 \% \mathrm{CO}_{2}, 5 \%$ $\mathrm{H}_{2}$, and $90 \% \mathrm{~N}_{2}$ with temperature held constant at $25^{\circ} \mathrm{C}$ with no light. Embryos were first removed from their normoxic medium, transferred into the chamber through an air lock, and placed in anoxic medium that had been previously bubbled with $\mathrm{N}_{2}$ gas for 30 min and equilibrated overnight to conditions in the anoxic chamber. Samples were collected as biological replicates consisting of five embryos each ( $N=4$ for DII, WS 36 , and WS $40 ; N=3$ for WS 42 ).

\section{Pharmacological inhibition of GABA synthesis and degradation}

Three inhibitors of canonical GABA synthesis and degradation were used (Fig 2.2A). Ethyl ketopentenoate (EKP) is a lipid-permeable and specific inhibitor of glutamate decarboxylase (GAD) (Zhang et al., 2017). Inhibition of GAD occurs through binding of EKP to the native glutamate active site of both isoforms of GAD (GAD67 and GAD65). EKP was synthesized and gifted to us from Dr. Peter A.M. de Witte and his lab (KU Leuven, Leuven, Belgium). Vigabatrin (vinyl-yaminobutyric acid) (V8261, Sigma Aldrich, St. Louis, MO, USA) is a selective, irreversible inhibitor of GABA transaminase (GABA-T; ABAT), the major enzyme that synthesizes the first step in the metabolic degradation of GABA (Ben-

Menachem, 2011; Qume and Fowler, 1997; Sloley et al., 1994; Wu et al., 2001). N,N-diethylaminobenzaldehyde (DEAB) (D86256, Sigma Aldrich, St. Louis, MO, 
USA) is a selective inhibitor of aldehyde dehydrogenase isoenzymes (Koppaka et al., 2012; Morgan et al., 2015). Succinic acid semialdehyde dehydrogenase (SSADH) is part of the aldehyde dehydrogenase 5 family (ALDH5A1), a target of DEAB (Morgan et al., 2015). Embryos of $A$. limnaeus are generally highly impermeable to external substances. Consequently, higher concentrations of inhibitors and the addition of dimethyl sulfoxide (DMSO) in the embryo medium are used to achieve biologically active concentrations within embryos (Podrabsky and Hand, 2000; Pri-Tal et al., 2011; Woll and Podrabsky, 2017). Stocks of pharmacological inhibitors were prepared in DMSO and diluted in embryo medium without antibiotics, yielding a final concentration of $1 \%$ DMSO. The final concentrations of inhibitors used in the experiments were: 400 and $800 \mu \mathrm{mol} \mathrm{I}^{-1}$ EKP; $1 \mathrm{mmol} \mathrm{I}^{-1}$ Vigabatrin; $167 \mu \mathrm{mol} \mathrm{I}^{-1}$ DEAB. Preliminary range-finding experiments were used to determine these concentrations in addition to observed concentrations used in the literature. The potential toxicity of each of the inhibitors was tested on WS 36 embryos. Embryos were treated with each of the inhibitors in normoxia and monitored for at least $7 \mathrm{~d}$ with daily media changes to observe any developmental defects or mortality. For anoxic exposures, groups of 15 WS 36 embryos $(N=3)$ were placed in clear 12-well polystyrene plates (CytoOne, CC7682-7512, USA Scientific, Ocala, FL, USA) and pre-exposed to embryo medium containing each inhibitor for $2 \mathrm{~h}$ at $25^{\circ} \mathrm{C}$ without light. Embryos were then transferred into the anoxic chamber where fresh anoxic embryo medium containing each inhibitor was replenished. Concentrated stock solutions (EKP, Vigabatrin: $100 \mathrm{mmol} \mathrm{I}^{-1}$; DEAB: $25 \mathrm{mmol}^{-1}$ ) of each inhibitor were 
prepared under anoxic conditions and stored at $-80^{\circ} \mathrm{C}$. These stocks were diluted daily within the anoxic chamber to make fresh embryo medium, which was changed daily. Embryos were monitored daily for survival until $100 \%$ mortality was reached. A separate plate of embryos was used for metabolite sampling purposes and embryos were sampled at $0,7,14$, and $30 \mathrm{~d}(N=3)$. Each replicate contained five embryos. To determine if a non-canonical pathway through polyamine metabolism was contributing to GABA production during anoxia, embryos were treated with amiloride. Amiloride is an inhibitor of diamine oxidase, the enzyme that catalyzes the penultimate step in GABA production by converting putrescine to 2-aminobutanal (Fig 2.2A). Amiloride $\left(100 \mu \mathrm{mol} \mathrm{I}^{-1}\right)$ treated embryos were only sampled at 0 and $8 \mathrm{~d}(N=4)$.

\section{Metabolite extraction}

Metabolites were extracted from whole embryos following previously detailed protocols (Podrabsky and Hand, 1999) and briefly described here. Samples were removed from $-80^{\circ} \mathrm{C}$ and immediately homogenized $(1: 7 \mathrm{w} / \mathrm{v})$ in ice-cold $1 \mathrm{~mol} \mathrm{I}^{-1}$ perchloric acid containing $5 \mathrm{mmol} \mathrm{I}^{-1}$ EDTA. Samples were then subjected to centrifugation at $16,000 \mathrm{~g}$ for $15 \mathrm{~min}$ at $4^{\circ} \mathrm{C}$ to pellet macromolecules and other insoluble cellular components. Supernatants were removed and neutralized with $9 \%$ volume of ice-cold $5 \mathrm{~mol} \mathrm{I}^{-1} \mathrm{~K}_{2} \mathrm{CO}_{3}$ and perchlorate precipitates were removed by centrifugation at $16,000 \boldsymbol{g}$ for $30 \mathrm{~min}$ at $4^{\circ} \mathrm{C}$. Supernatants were then transferred to new tubes and frozen at $-80^{\circ} \mathrm{C}$ until GABA and lactate determinations were performed. 


\section{Y-aminobutyric acid (GABA) determination}

GABA levels were enzymatically measured using a spectrophotometric assay that was modified from previously established protocols using a 96 well plate format (Ippolito and Piwnica-Worms, 2014; Tsukatani et al., 2005). Samples $(10 \mu \mathrm{l})$ were added to wells of a black clear bottom 96 well plate (Corning ${ }^{\mathrm{TM}}$ $3603,07-200-565$, Fisher Scientific, Hampton, $\mathrm{NH}$, USA), followed by addition of $90 \mu \mathrm{l}$ of a freshly prepared reaction mix containing: $0.1 \mathrm{~mol} \mathrm{I}^{-1} \mathrm{Tris}-\mathrm{HCl}(\mathrm{pH} 8.8)$, $10 \mathrm{mmol} \mathrm{I}^{-1} \mathrm{DTT}, 1.4 \mathrm{mmol} \mathrm{I}^{-1} \mathrm{NADP}, 2.0 \mathrm{mmol} \mathrm{I}^{-1} \alpha$-ketoglutarate, and $0.126 \mathrm{U}$ $\mathrm{ml}^{-1}$ GABase (G7509, Sigma Aldrich, St. Louis, MO, USA). Change in absorbance was recorded every $60 \mathrm{~s}$ for $90 \mathrm{~min}$ at $340 \mathrm{~nm}\left(A_{340}\right)$ to monitor NADPH production (Tecan Infinity M200Pro, Männendorf, Switzerland). Because any endogenous SSAL found in the sample would give false GABA levels, an inhibitor of GABA-T, 2-aminoethyl hydrogen sulfate (2-AEHS) (TCS0445, VWR, Radnor, PA, USA), was used to account for any signal resulting from endogenous SSAL being converted to GABA in the assay. An inhibitor reaction mix was created containing $50 \mathrm{mmol}^{-1} 2$-AEHS. The reaction mix was allowed to incubate for $10 \mathrm{~min}$ following addition of GABase but before addition of the sample to ensure sufficient time for GABA-T inhibition. The final concentration of GABA was determined by subtracting the amount of GABA measured in the presence of inhibitor from the total quantity of GABA detected in the assay. Both GABA and SSAL standards were used to calculate concentrations using a standard curve. A standard curve was generated for each assay and $A_{340}$ increased in a linear fashion with increasing GABA concentrations $\left(R^{2}=0.99 \pm\right.$ 
$0.001, N=41$ assays). SSAL concentrations were generally low in the samples and never represented more than $5 \%$ of the total GABA measured.

\section{Lactate determination}

Lactate levels were enzymatically measured following a previously detailed protocol, but modified for use in a 96 well plate format (Gleeson, 1985). Each well contained the following: $218 \mu$ of Glycine $\left(0.6 \mathrm{~mol} \mathrm{I}^{-1}\right)$-Hydrazine $(0.5$ mol l-1) buffer ( $\mathrm{pH}=9.2), 25 \mu \mathrm{l} 27 \mathrm{mmol} \mathrm{I}^{-1} \mathrm{NAD}^{+}, 5 \mu$ of lactate standard or sample, and $2.5 \mu \mathrm{l} 14 \mathrm{U} \mathrm{ml}^{-1}$ lactate dehydrogenase (LDH; L2625, Sigma Aldrich, St. Louis, MO, USA). The addition of LDH catalyzed the oxidation of lactate to pyruvate and the associated production of NADH was monitored spectrophotometrically at $A_{340}$ for $60 \mathrm{~min}$ (Tecan Infinity M200Pro). The final concentration of lactate was determined by interpolating sample $A_{340}$ to the lactate standard curve $\left(R^{2}=0.99 \pm 0.0004, N=25\right.$ assays $)$.

\section{Glutamate decarboxylase (GAD) activity}

Glutamate decarboxylase (GAD) activity was measured following a previously detailed protocol with modifications (Wolf and Klemisch, 1991). Whole embryos in groups of $13(N=4)$ from 4 developmental stages (DII, WS 36, WS 40 , and WS 42) were removed from $-80^{\circ} \mathrm{C}$ and immediately mechanically homogenized with a Teflon pestle $(1: 3 \mathrm{w} / \mathrm{v})$ in ice-cold $10 \mathrm{mmol} \mathrm{I}^{-1}$ potassium phosphate buffer ( $\mathrm{pH} 7.0)$ containing $1 \mathrm{mmol} \mathrm{I}^{-1}$ 2-(2-aminoethyl)isothiourea dihydrobromide (AET), $0.1 \%$ Triton X-100, and $250 \mu \mathrm{mol} \mathrm{I}^{-1}$ pyridoxal $5^{\prime}-$ 
phosphate (PLP). Samples were then sonicated on ice at $25 \%$ amplitude for $15 \mathrm{~s}$ to disrupt cells (Branson Digital Sonifier, S-450D, 1/8" SF 150 microtip probe). Samples were then subjected to centrifugation at $5,000 \mathrm{~g}$ for $30 \mathrm{~min}$ at $4^{\circ} \mathrm{C}$. Supernatants containing GAD were removed and frozen at $-80^{\circ} \mathrm{C}$ until the enzyme assay could be run. Preliminary experiments showed no difference in GAD activity between fresh and previously frozen samples. Samples were removed from $-80^{\circ} \mathrm{C}$ and allowed to thaw at room temperature for 5 min and then placed on ice for $10 \mathrm{~min}$. Samples were then subjected to centrifugation at $5,000 \mathrm{~g}$ for $30 \mathrm{~min}$ at $4^{\circ} \mathrm{C}$ prior to measuring activity. Supernatants were removed and $10 \mu \mathrm{l}$ were mixed with $10 \mu \mathrm{l}$ of GAD-substrate solution $\left(0.1 \mathrm{~mol} \mathrm{l}^{-1}\right.$ potassium phosphate buffer, $\mathrm{pH} 8.0$, containing $50 \mathrm{mmol} \mathrm{I}^{-1}$ glutamate, $250 \mu \mathrm{mol} \mathrm{I}^{-1} \mathrm{PLP}$, and $0.4 \% 2$-mercaptoethanol). Samples were incubated at $25^{\circ} \mathrm{C}$ without light for $0,6,12$, and $24 \mathrm{~h}$. Samples $(N=4)$ containing $10 \mu \mathrm{l}$ water and $10 \mu \mathrm{l}$ GADsubstrate solution were used as negative controls. Reactions were terminated by addition of $10 \mu \mathrm{l} 0.25 \mathrm{~mol} \mathrm{I}^{-1} \mathrm{HCl}$ and then placed on ice for $5 \mathrm{~min}$. Samples were then frozen at $-80^{\circ} \mathrm{C}$ until GABA concentrations were determined as described above. To determine the efficacy of our assay in measuring GAD activity, we measured activity in adult $A$. limnaeus brains that were dissected from 2 males and pooled together. Brain samples $(N=1)$ were subjected to the same sample preparation as embryos. Following the addition of GAD-substrate solutions ( $\mathrm{pH}$ $6.0,7.0,8.0$, or 9.0 ), samples were incubated at $25^{\circ} \mathrm{C}$ without light for $0,0.75$, 1.5 , and $3 \mathrm{~h}$ and GABA accumulation was measured as described above. 


\section{RNAseq data and identification}

Gene transcripts associated with GABA production, signaling, degradation, transport, and binding, as well as polyamine metabolism, lactate production and transport, and ethanol production were identified from the $A$. limnaeus genome annotation version 100 in the NCBI GenBank database (Wagner et al., 2018). Transcript expression data were obtained from datasets deposited in GenBank (Bioproject PRJNA272154). Data for early embryos developing at $20^{\circ} \mathrm{C}$ that will enter into DII $(N=3)$ are from Romney et al. (2018). Data for DII and post-DII embryos are from embryos incubated at $25^{\circ} \mathrm{C}$ and exposed to short-term anoxia. The data were collected as outlined previously (Riggs and Podrabsky, 2017). Briefly, DII, WS 36, and WS 40 embryos were sampled after 4 and $24 \mathrm{~h}$ of anoxia and after 2 and $24 \mathrm{~h}$ of aerobic recovery $(N=$ 4 for DII and WS 36; $N=6$ for WS 40). WS 42 embryos were sampled at 2 and $6 \mathrm{~h}$ of anoxia followed by 2 and $24 \mathrm{~h}$ of aerobic recovery $(N=4)$. RNA sequencing libraries were constructed using the Illumina TruSeq RNA Sample Preparation Kit (v2, Illumina, San Diego, CA, USA) following the manufacturer's instructions. Libraries were sequenced (100 bp paired-end reads) on a HiSeq 2000 (Illumina) instrument as outlined in Romney and Podrabsky (2017). Some of these data have been previously published (Wagner et al., 2018).

\section{Statistical analysis}

Graphical and statistical analyses were performed using Prism 8.0 software (GraphPad, La Jolla, CA, USA) and SPSS software (IBM., v.26.0, 
Armonk, NY, USA). $L T_{50}$ of embryos exposed to inhibitors in anoxia were calculated by probit regression analysis (Chapman et al., 1995) using SPSS software. Where appropriate, analysis of variance (ANOVA), analysis of covariance (ANCOVA), unpaired t-test, correlation analysis, linear regression, segmental linear regression, or exponential one-phase decay nonlinear regression were used. Nonlinear and segmental linear regressions reported only include coefficient of determination $\left(R^{2}\right)$ values, as probability $(P)$ values cannot be calculated. To support whether slopes calculated are significantly non-zero, linear regressions were initially performed and were significant $(P<0.05)$. Tukey's honestly significant difference (HSD) test and Dunnett's test were used for post-hoc comparisons, where applicable. Statistical significance was set to $P$ $<0.05$ for all comparisons.

\section{Results}

\section{Long-term anoxia exposure and recovery}

\section{Lactate}

All developmental stages of $A$. limnaeus responded to anoxia by accumulating significant amounts of lactate (Fig 2.1B; one-way ANOVA, $P<$ 0.0001). To account for clear differences in initial and final rates of production, segmental linear regressions were performed for each developmental stage (Fig 2.1B; Table 2.1). Initial rates were higher than final rates for each stage of embryo (Table 2.1). Rates were lowest in DII and increased with further post-DII development (one-way ANOVA with Tukey's HSD, $P<0.01$ ). WS 36 embryos 
accumulated the highest amount of lactate during anoxia (Fig 2.1B; $23.6 \pm 1.09$ mmol kg-1 embryo; mean \pm SEM, $N=4)$, followed by WS 40 embryos $(18.7 \pm$ $3.07 \mathrm{mmol} \mathrm{kg}^{-1}$ embryo). However, WS 40 embryos accumulated lactate at a rate seven times faster than WS 36 embryos (Table 2.1). We observed a significant correlation between initial rates of lactate accumulation and $\mathrm{LT}_{50}$ in anoxia (Fig 2.2A). The relationship between initial rates of lactate accumulation and $L T_{50}$ in anoxia reported here on DII and post-DII embryos is similar to those from previous experiments on pre-DII and DIl embryos (Fig 2.2A; ANCOVA, $P=$ 0.1886) (Podrabsky et al., 2007). For those embryos that recover from anoxia, lactate slowly returned back to initial levels after several days to a week (DII, WS 36 and WS 40; Dunnett's post-hoc, $P<0.05)$.

\section{GABA}

There was significant accumulation of GABA in DII, WS 36, and WS 40 embryos, while WS 42 embryos showed no significant accumulation (Fig 2.1B; one-way ANOVA, $P<0.0001$ for DII, WS 36 and WS 40; $P=0.2286$ for WS 42). There were clear initial and final rates of GABA accumulation in DII, WS 36, and WS 40 embryos, so segmental linear regressions were performed (Fig 2.1B; Table 2.1). Regression analysis of data for WS 42 embryos confirmed a slope that was not statistically different from zero (Table 2.1). Initial rates of GABA accumulation were higher than final rates in DII and WS 36 embryos (Table 2.1). In contrast, final rates were higher than initial rates in WS 40 embryos (unpaired t-test, $P<0.05)$. Rate of GABA accumulation was lowest in DIl embryos and 
increased with post-DII development in WS 36 and WS 40 embryos. There was no relationship found between rates of initial or final GABA accumulation and $\mathrm{LT}_{50}$ in anoxia (Fig 2.2B; correlation analysis, initial: $r=0.10, P=0.8951$; final: $r$ $=-0.34, P=0.6561)$. An inverse relationship was found in the ratio of lactate to GABA accumulated at the $L T_{50}$ when compared to the $L T_{50}$ of each stage (Fig 2.2C). During aerobic recovery from anoxia, GABA slowly returned to initial levels in DII and WS 40 embryos (Dunnett's post-hoc, $P<0.05$ ).

\section{Toxicity of pharmacological inhibitors in normoxia}

In normoxia, WS 36 embryos incubated in GAD inhibitor EKP $\left(400 \mu \mathrm{mol} \mathrm{I}^{-}\right.$ ${ }^{1}$ ), GABA-T inhibitor vigabatrin, SSADH inhibitor DEAB, and DAO inhibitor amiloride survived, developed normally, and had developmental rates that were indistinguishable from those of control embryos (data not shown). In contrast, embryos exposed to $800 \mu \mathrm{mol} \mathrm{^{-1 }}$ EKP showed mortality following $5 \mathrm{~d}$ of exposure (Fig 2.3). However, it should be noted that embryos continue developing in normoxia, which is not the case during exposure to anoxia. Additionally, embryos exposed to $800 \mu \mathrm{mol} \mathrm{I}^{-1}$ EKP survived in anoxia at WS 36 significantly longer than developing embryos exposed to $800 \mu \mathrm{mol} \mathrm{I}^{-1} \mathrm{EKP}$ in normoxia (Fig 2.5B,C;

Fig 2.3). Exposure to $800 \mu \mathrm{mol} \mathrm{l}^{-1}$ EKP starting at WS 40 led to mortality after $2 \mathrm{~d}$ (Fig 2.3). These data are interpreted to indicate stage-specific toxicity of EKP in WS 39 and later embryos and likely illustrate a necessity for GABA in late development. 


\section{Inhibition of GABA synthesis and degradation in anoxia}

Ethyl ketopentenoate (EKP)

Ethyl ketopentenoate, a potent inhibitor of GAD, exhibited dosagedependent reductions in GABA production during anoxia in WS 36 embryos (Fig 2.4B,C). GABA levels were significantly lower after $14 \mathrm{~d}_{\text {in }} 400 \mu \mathrm{mol} \mathrm{I}^{-1}$ treated embryos when compared to controls, while levels were significantly lower after only $7 \mathrm{~d}$ in $800 \mu \mathrm{mol} \mathrm{I}^{-1}$ treatments (Fig 2.4B,C; two-way ANOVA with Tukey's HSD, $P<0.05)$. Embryos exposed to $400 \mu \mathrm{mol} \mathrm{I}^{-1}$ EKP produced GABA at a rate $42 \%$ slower than control embryos; whereas, embryos exposed to $800 \mu \mathrm{mol} \mathrm{I}^{-1}$ EKP produced GABA at a rate $52 \%$ slower than controls. Interestingly, exposure to either concentration of EKP led to no changes in rates of lactate accumulation (Fig 2.4D,E; two-way ANOVA with Tukey's HSD, $P>0.05$; ANCOVA, $P>0.05$ ). However, exposure to both dosages of EKP led to decreased survival in anoxia (Fig 2.5A,B). When the survival data were used to calculate $L T_{50}$ values, embryos exhibited a $37 \%$ and $44 \%$ reduction in survival compared to controls, with $\mathrm{LT}_{50}$ values of $44 \pm 4$ and $39 \pm 2 \mathrm{~d}$ (mean \pm SEM, $N=3$ ) in anoxia for 400 and $800 \mu \mathrm{mol} \mathrm{I}^{-1}$ treatments, respectively (Fig 2.5C). Despite having similar mean $\mathrm{LT}_{50}$ values (one-way ANOVA, $P=0.78$ ), $800 \mu \mathrm{mol} \mathrm{I}^{-1}$ treated embryos did not survive as long as $400 \mu \mathrm{mol} \mathrm{I}^{-1}$ treated embryos (Fig 2.5B,C). Embryos treated with $400 \mu \mathrm{mol} \mathrm{I}^{-1}$ reached $100 \%$ mortality after $62 \mathrm{~d}$, while it only took $51 \mathrm{~d}$ for $800 \mu \mathrm{mol} \mathrm{I}^{-1}$ treated embryos. 
Vigabatrin

Vigabatrin, an inhibitor of GABA-T, increased GABA levels in WS 36 embryos exposed to anoxia after 14 d compared to control embryos (Fig 2.4C; two-way ANOVA with Tukey's HSD, $P<0.05)$. GABA accumulated at a rate $23 \%$ faster than controls. Lactate accumulation was not affected by vigabatrin (Fig 2.4E; two-way ANOVA with Tukey's HSD, $P>0.05$; ANCOVA, $P>0.05$ ). Vigabatrin decreased survival of anoxic embryos by $20 \%\left(\mathrm{LT}_{50}=56 \pm 0.5 \mathrm{~d}\right.$; mean $\pm \mathrm{SEM}, N=3$; Fig 2.5B,C).

$N, N$-diethylaminobenzaldehyde (DEAB)

DEAB, an inhibitor of SSADH, increased GABA levels in WS 36 embryos exposed to anoxia after $30 \mathrm{~d}$ compared to control embryos (Fig 2.4B, two-way ANOVA with Tukey's HSD, $P<0.05)$. GABA accumulated at a rate $26 \%$ faster than controls. DEAB had no effect on rates of lactate accumulation (Fig 2.4D; two-way ANOVA with Tukey's HSD, $P>0.05$; ANCOVA, $P>0.05)$. DEAB decreased survival of anoxic embryos (Fig $2.5 \mathrm{~B}$ ), leading to a $44 \%$ reduction in $\mathrm{LT}_{50}(39 \pm 4 \mathrm{~d}$; mean $\pm \mathrm{SEM}, N=3 ;$ Fig $2.5 \mathrm{C})$.

\section{Amiloride}

Amiloride, an inhibitor of DAO, did not affect GABA levels in WS 36 embryos exposed to anoxia for $8 \mathrm{~d}$ (Fig 2.6). 


\section{Glutamate decarboxylase (GAD) activity during development}

Significant rates of GABA synthesis were observed in homogenates of adult brain tissue incubated at $\mathrm{pH} 7-9$, confirming the utility of this assay for estimating GAD activity in homogenates of $A$. limnaeus (Fig 2.7; regression analysis, $P<0.005)$. We observed low, but significantly greater than zero, rates of GAD activity in post-DIl embryo homogenates (Fig 2.8A). No significant GAD activity was detected in DII embryos after $24 \mathrm{~h}$ of incubation (Fig 2.8A). Despite the low observed GAD activity, this capacity for GABA production is sufficient to account for the GABA produced in whole embryos during anoxia. Observed GAD activity provides reasonable prediction of initial GABA accumulation during anoxia (LT50/4) for DII and WS 36 embryos (Fig 2.8B; unpaired t-test, $P>0.05$ ). In contrast, the capacity for GABA production by GAD is significantly greater than needed in WS 40 and WS 42 embryos (unpaired t-test, $P<0.05$ ). Despite observing the highest enzymatic capacity for GAD activity, WS 42 embryos did not produce significant levels of GABA during anoxia (Fig 2.1B). There is a significant negative relationship between $\mathrm{LT}_{50}$ and GAD activity (Fig 2.8C).

\section{RNAseq}

GABA pathway

Early developing embryos had generally higher levels of transcripts involved in GABA synthesis and degradation, which tended to decrease and level off as they approached DII (Fig 2.9A). DIl embryos observed relatively constant expression of these transcripts during anoxia and recovery. Interestingly GAD2 
expression was highest in DII, whereas it was absent or low in expression in all other stages. WS 36 embryos exhibited highest expression of GAD1 in post-DII embryos, which increased with short term anoxia exposure and decreased with aerobic recovery. Transcripts for GABA-T decreased with anoxia and recovery, but were still expressed (> 1000 FPKM). SSADH transcripts levels were maintained during anoxia and recovery in DII and post-DII stages. WS 40 embryos have relatively high GAD1 and GABA-T expression. The overall expression pattern for GABA synthesis and degradation in WS 42 embryos was relatively high and constant during anoxia, despite not producing GABA. However, expression of GAD transcripts increased during aerobic recovery. GAD enzyme activity (Fig 2.8C) is inversely related to transcript expression patterns for GAD enzymes in post-DII embryos.

\section{Polyamine metabolism}

A number of transcripts involved in polyamine metabolism were abundant in all embryonic stages (Fig 2.9B). The most abundant transcripts (SAT1, ODC1, and $A L D H 9 A 1$ ) remained high throughout development. A transcript that encodes a diamine oxidase (AOC2) was highest in WS 20 embryos and decreased with development towards DII, where it then increased in abundance and remained high during DII and post-DII development. 


\section{Lactate and ethanol pathways}

Transcripts for lactate dehydrogenases (LDH) were abundant in all stages of embryos (Fig 2.9C). LDH B was the primary isoform expressed in early embryos, while both the A and B isoforms were expressed in DII. For post-DII embryos, LDH B was the primary transcript expressed with LDH A appearing in WS 36 embryos during recovery from anoxia. Transcripts for proteins involved in ethanol production were low in DII and post-DII embryos, with the most abundant transcripts (Fig 2.9C; $P D H B, P D K 2 A, D L D$, and $A D H 3$ ) showing similar expression patterns in all stages, with the exception of an ADH1-like transcript being highly expressed in WS 40 embryos.

\section{GABA and lactate transporters}

GABA transporters (GAT) were absent or expressed in low levels during early development (Fig 2.9D). In contrast, transcripts for GAT1 and GAT3 paralogs were highly expressed in DIl embryos. These same transcripts were induced during anoxia and aerobic recovery in WS 36 embryos. WS 40 embryos uniquely only expressed GAT2 paralogs, whereas WS 42 embryos expression patterns were similar to Dll embryos, but at lower levels. An abundance of monocarboxylate transporters (MCTs) were expressed in embryos of $A$. limnaeus (Fig 2.9D). Early developing embryos displayed the highest expression of MCTs, primarily expressing MCT1-like and MCT7-like transcripts, which were highest in WS 20 embryos and decreased with development towards DII. Generally, DII embryos exhibited more MCT expression than post-DIl embryos. 


\section{GABA receptors and cotransporters}

The A. limnaeus genome contains an abundance of $\mathrm{GABA}_{\mathrm{A}}$ receptor subunit variants compared to relatively few $G A B A_{B}$ receptor subunit variants (Fig 2.10). Despite the high expression of transcripts involved in GABA synthesis and degradation, transcripts for GABA signaling were predominantly absent in early developing embryos, with the exception of a single $G A B A_{A} R$ subunit-p-3-like transcript. In contrast, DIl embryos expressed the highest diversity of GABA receptor subunits. WS 36 embryos had relatively low amounts of expression prior to anoxia, which consistently increased during anoxia and aerobic recovery. WS 40 embryos had similar expression to early developing embryos, with most receptors being minimally expressed besides $\mathrm{GABA}_{A} R$ subunit-p-3-like. WS 42 embryos had consistent expression of several receptors that generally matched the receptors expressed in DIl embryos, some of which increased during recovery from anoxia. $\mathrm{GABA}_{\mathrm{A}}$ receptor-associated proteins were highly expressed in all stages (Fig 2.10). The highest expression of $\mathrm{GABA}_{\mathrm{A}}$ receptorassociated proteins was found in WS 20 embryos. Cotransporters (NKCC and KCC) were observed in all stages of embryos (Fig 2.10). KCC1 expression was low early in development, but increased as embryos approached DII and remained constant during post-DII development. Interestingly, NKCC2 was highly expressed in DII embryos, but barely expressed in all other stages. 


\section{Age of female on GABA and lactate accumulation}

Sampling of embryos spanned the lifespan of $A$. limnaeus and across generations. Due to differences in GABA and lactate levels observed between experiments, we explored the relationship between female age and rates of GABA and lactate accumulation in anoxic WS 36 embryos. No correlation was found between the rate of GABA accumulation and female age (Fig $2.11 \mathrm{~A} ; R^{2}=$ $0.07, P=0.67)$. Interestingly, embryos from younger females produced lactate during anoxia at a faster rate than embryos produced by older females (Fig 2.11B; $\left.R^{2}=0.97, P<0.01\right)$.

\section{Discussion}

The impressive survival of anoxia in A. limnaeus embryos appears to rely fundamentally on the same metabolic pathways as anoxia-sensitive organisms with lactate as the major product of anaerobic glycolysis. Consistent with previous data for early embryos of $A$. limnaeus (Podrabsky et al., 2007) and a variety of other organisms (Hand, 1998), survival of anoxia in DII and post-DII embryos is proportional to the level of metabolic depression achieved. This point is illustrated well by the high negative correlation between rate of lactate accumulation and survival of anoxia as estimated by the $L T_{50}$. Thus, the fundamental key to long-term survival of anoxia appears to be the limiting of lactate accumulation.

Lactic acidosis is a major factor in contributing to cell death in low oxygen environments (Kubasiak et al., 2002). Fish embryos are a closed system, which 
is especially important because metabolic end-products, such as lactate, have no means of exiting the embryo. In the western painted turtle, lactic acidosis is buffered by the turtle's mineralized shell (Jackson, 1997; Jackson et al., 2000; Jackson and Heisler, 1982). The internal pH of $A$. limnaeus embryos has never been measured. Although the embryos have a high tolerance of a wide range of external pH conditions and can survive for about a month at pH 2 (Podrabsky et al., 2016), it is unclear whether embryos have high capacity to buffer the acidosis of lactate or if they are able to tolerate a low internal $\mathrm{pH}$. The robust expression of several lactate transporters suggests lactate is likely being shuttled within the embryo. A likely destination for lactate is the yolk. Shuttling lactate into the yolk may prevent embryonic cells from experiencing severe acidosis. However, additional work is needed to explore the buffering capacity of the yolk and possible compartmentalization of metabolic pathways between the yolk and embryonic cells that may support anaerobic metabolism.

The general characterization of lactate as a poor anaerobic end-product may be unwarranted. In appropriate quantities, lactate has been seen to have important roles in regulating gene expression through epigenetic mechanisms. For example, lactate has been shown to inhibit histone deacetylase activity (HDAC), which can have genome wide effects (Latham et al., 2012). In addition, recent evidence suggests that histones can be post-translationally modified by the addition of lactate (lactylation) which induces change in gene expression through modification of chromatin structure (Zhang et al., 2019). Lactate has also been found to have a neuroprotective role, preventing excitotoxic cell death and 
insults from cerebral ischemia (Berthet et al., 2009; Jourdain et al., 2016). It was recently shown that human neuroblastoma cells (SH-SY5Y) and C. elegans pretreated with lactate promoted oxidative stress resistance and resulted in longterm stress resistance and longevity (Tauffenberger et al., 2019). Thus, it is possible that lactate is a necessary and conserved part of the vertebrate response to anoxia, and when coupled with the proper mechanisms for metabolic suppression can be an effective means of supporting anaerobic metabolism for long-term survival.

Previous studies in Crucian carp suggest that GABA may play a role in suppressing metabolism during anoxia as indicated by a near three-fold increase in ethanol (the major anaerobic end-product) production when fish were injected with isoniazid, an inhibitor of GAD (Nilsson, 1992). In A. limnaeus, GABA synthesis and lactate production appear to be independent as evidenced by no change in lactate accumulation with inhibition of GAD, GABA-T, or SSADH. While it is possible that inhibition of GABA synthesis could be affecting some other major metabolic pathway such as ethanol production, it is unlikely given the rather extensive metabolomics studies performed on $A$. limnaeus, the generally low levels of transcripts for proteins in this pathway, and the fact that previous efforts have failed to detect ethanol production in embryos (Podrabsky and Hand, 1999). Thus, we conclude that GABA is not playing a general role in suppression of metabolism during anoxia in embryos of $A$. limnaeus.

Decreased survival when GABA-T and SSADH are inhibited suggests that at least part of the GABA produced during anoxia is being metabolized and 
shuttled into the TCA cycle. Because GABA degradation can lead to ammonia consumption, NADH oxidation, and GTP production (Goldlust et al., 1995), this response could be beneficial for supporting embryonic metabolism during exposures to anoxia. The rate of lactate accumulation appears to decrease over long-term anoxia, which may indicate a shift in reliance to GABA metabolism.

Although GABA metabolism appears to be critical for long-term survival in anoxia, the innate capacity for high GAD activity does not correspond to anoxia tolerance. We hypothesized that embryos from anoxia-tolerant stages would have higher basal GAD enzymatic activity than anoxia-sensitive stages.

However, we saw an inverse relationship between normoxic GAD activity and anoxia tolerance. Despite having the highest capacity to make GABA, WS 42 embryos produced negligible GABA in anoxia. This is contrary to what is seen with LDH capacity in A. limnaeus embryos, where there is an increase in LDH capacity with post-DII development that translates to high LDH activity during anoxia (Chennault and Podrabsky, 2010).

Anoxia-induced GABA synthesis is not unique to $A$. limnaeus embryos (Nilsson and Lutz, 2004; Nilsson et al., 1991; Podrabsky et al., 2007). Despite previous knowledge of GABA accumulation in anoxia, this is the first study to directly relate GABA metabolism to survival of $A$. limnaeus embryos in anoxia. We found that inhibition of enzymes responsible for both GABA synthesis (via GAD) and degradation (via GABA-T and SSADH) led to decreased survival (20$44 \%$ reduced $\mathrm{LT}_{50 \mathrm{~S}}$ ) in the most anoxia-tolerant developmental stage (WS 36). Inhibition of enzymes that degrade GABA (GABA-T and SSADH) lead to 
increased accumulation of GABA, and decreased survival; thus, increased concentrations of GABA do not necessarily translate to longer survival. These data suggest a role for GABA as a metabolic intermediate supporting anoxic metabolism, and not a simple end-product. Interestingly, the effects of decreased GABA production on survival are not obvious until after 3-4 weeks of anoxia, suggesting a role in long-term rather than short-term survival. Further, doubling the dose of EKP to block GABA production had only a marginal effect on the $\mathrm{LT}_{50}$, but resulted in a much more rapid time to $100 \%$ mortality. Thus, we conclude that GABA synthesis plays a critical role in long-term anoxia tolerance as a metabolic intermediate of critical importance.

The abundance and diversity of GABA transporters expressed in $A$. limnaeus embryos suggest GABA may be shuttled within the embryo or within cells after production. The expression of high levels of GABA transporters in DII embryos and anoxia-induced elevation in transcripts of GABA transporters in WS 36 embryos suggest an importance for GABA shuttling during anoxia. Several GABA transporters remain high or continue to increase during aerobic recovery in DII and WS 36 embryos. One possibility is that GABA is being transported into the yolk. Alternatively, GABA may be synthesized in the yolk and transported to the developing embryo. This is consistent with a lack of GABA production in two established anoxia-tolerant cell lines of $A$. limnaeus (PSU-AL-WS40NE, WS36-2 cells; unpublished data). Another possibility is that only specific cells within the embryo produce GABA, and the two neural-derived cell lines isolated are not responsible for GABA production. Nevertheless, preliminary evidence suggests 
GABA present in both the yolk and cellular fractions of embryos (unpublished). The internal pH of embryos and the localization of lactate and GABA during anoxia and recovery from anoxia are currently unknown and need to be resolved to fully understand the physiology of GABA production in this species.

The main function of GABA in adult vertebrates is as a neurotransmitter. The diversity of GABA receptor subunits expressed in $A$. limnaeus embryos support the potential for GABA as an early neurotransmitter in this species. GABA has been found to provide excitatory actions in the developing vertebrate nervous system, but conversely, typically functions as an inhibitory neurotransmitter in adults (Ben-Ari, 2002). The switch from excitatory to inhibitory transmission occurs due to delayed expression of $\mathrm{K}^{+}-\mathrm{Cl}^{-}$-coupled-co-transporters (KCC). Transcript expression data support GABA acting as an inhibitory transmitter during $A$. limnaeus development due to the rise in KCC transcripts as embryos develop to DII and beyond. This may represent a unique developmental adaptation to anoxia tolerance in $A$. limnaeus.

Increased GABA levels are thought to protect the brain of many organisms from excitotoxic cell death during hypoxic and anoxic conditions (Lutz and Milton, 2004; Lutz and Nilsson, 2004; Nilsson and Lutz, 2004; Podrabsky et al., 2007). A main factor that differentiates anoxia-tolerant and anoxia-sensitive species is their ability to maintain glutamate homeostasis in response to low oxygen levels (Nilsson and Renshaw, 2004). The ability to convert an excitatory neurotransmitter (glutamate) to an inhibitor neurotransmitter (GABA) may be beneficial to embryos trying to depress their metabolism and prevent excitotoxic 
cell death during anoxia. Increased endogenous GABA release during anoxia dampened excitatory potentials in turtle brain (Pamenter et al., 2011) and preventing GABA reuptake and metabolism in mammalian models supports neuroprotection during ischemia (Prentice, 2009; Schwartz-Bloom and Sah, 2001). Furthermore, turtle neurons treated with $G A B A_{A}$ and $G A B A_{B}$ receptor antagonists exhibited seizure-like effects and eventual cell death, similar to excitotoxic cell death in anoxic mammalian brains (Pamenter et al., 2011). Perhaps GABA is acting in a similar manner in A. limnaeus, thereby increasing the stimulation required to elicit an action potential. However, the accumulation of GABA in gross excess of that needed for neuroprotection in A. limnaeus (1000 times more than in other anoxia tolerant species), suggests multiple or novel functions. Localization of GABA receptors and transporters in embryos and their role in anoxia is worth further exploration. Functional tests through exposure of embryos to GABA receptor antagonists could lend insight into the neurotransmitter function of GABA in anoxia.

This is the first extensive study of long-term anoxia and aerobic recovery in A. limnaeus. Our findings suggest that embryos seemingly go unharmed and without developmental defect following long-term anoxia. However, recovery from anoxia appears to be slow with lactate and GABA levels not returning to pre-anoxia levels until after at least a week of aerobic recovery. This slow reduction of both lactate and GABA may be an adaptive and protective mechanism for supporting survival of long-term anoxia. A primary cause of mortality due to anoxic exposure is not the lack of oxygen itself, but ironically the 
reoxygenation event that occurs after (Bundgaard et al., 2020; Saikumar et al., 1998). A rapid increase in reactive oxygen species (ROS) in response to reinitiating aerobic respiration can lead to damaging of DNA, cell membranes, and proteins (Schieber and Chandel, 2014). In anoxia-sensitive mammals, reoxygenation is associated with overproduction of ROS from mitochondria, which often initiates programmed cell death (apoptosis). Although ROS has not been quantified in embryos of $A$. limnaeus, they possess a large antioxidant capacity that is likely to help protect against ROS production (Wagner et al., 2019). A surge in ROS production can likely be minimized by a slow increase in aerobic metabolism following recovery. The inhibitory and sedative effects of GABA on neurons may prevent a surge of synapses firing, thereby decreasing ROS production by slowly increasing aerobic metabolism. This would be consistent with observations of a slow return of a heartrate in WS 36 embryos following long-term anoxia exposure. One interesting possibility is the shunting of GABA into polyamine metabolism during transitions from anoxia to normoxia, or perhaps during severe hypoxia. GABA can be converted to putrescine through a pathway that utilizes the enzyme diamine oxidase (DAO), which consumes hydrogen peroxide and produces molecular oxygen in the process. This would be an excellent method to help prevent accumulation of ROS damage during periods of severe oxygen limitation. The expression of genes involved in this pathway would support this possible function for GABA during recovery from anoxia or during prolonged severe hypoxia, both conditions where elevated hydrogen peroxide production would be expected. Because DAO requires 
molecular oxygen or hydrogen peroxide to function, this pathway would be largely blocked during long-term anoxia allowing GABA to accumulate in preparation for use during periods of reoxygenation. In addition, this could be a mechanism for detoxification of ROS associated with episodic or brief reintroductions of oxygen. Consistent with this hypothesis, inhibition of DAO with amiloride has no effect on GABA accumulation during anoxia (Fig 2.6). Similarly, in the yeast Saccharomyces cerevisiae, the GABA shunt pathway has been shown to be critical in reducing ROS production and protecting against oxidative stress (Cao et al., 2013; Coleman et al., 2001). Though not yet investigated in animals, the conserved role of GABA across bacteria, yeast, plants, and animals in reduction of oxidative stress suggests this possibility in $A$. limnaeus. Perhaps GABA and lactate accumulated during anoxia aid in both surviving without oxygen and the reoxygenation event that follows. The combination of lactate and GABA serving as neuroprotective and antioxidant agents is worth exploring further.

Previous studies on $A$. limnaeus embryos have shown their extreme anoxia tolerance, (Meller et al., 2012; Podrabsky et al., 2007; Riggs and Podrabsky, 2017), but this is the first study to observe these metabolites in response to long-term anoxia across development and aerobic recovery. We show that GABA may have multiple functions in A. limnaeus embryos that could be contributing to survival during anoxia and recovery. Most importantly, we show that GABA is being synthesized and degraded using common pathways in vertebrates. Further exploration into the metabolism, signaling, and localization of 
GABA and lactate in A. limnaeus embryos will provide further insight into how anoxia-sensitive organisms can survive anoxic events and avoid ischemic injury. 
Table 2.1. Segmental linear regression analysis of GABA and lactate accumulation during exposure to anoxia

\begin{tabular}{|c|c|c|c|c|c|}
\hline & Parameter & WS 32/33 (DII) & WS 36 & WS 40 & WS 42 \\
\hline & Initial $\left(\mathrm{mmol} \mathrm{kg}^{-1}\right.$ embryo $\left.^{-1} \mathrm{~d}^{-1}\right)$ & 0.126 & 0.281 & 0.561 & a \\
\hline & $\mathrm{X}_{0}(\mathrm{~d})$ & 16 & 18 & 2 & a \\
\hline \multirow[t]{5}{*}{ GABA } & Final $\left(\mathrm{mmol} \mathrm{kg}^{-1}\right.$ embryo $\left.^{-1} \mathrm{~d}^{-1}\right)$ & 0.035 & 0.156 & 0.733 & a \\
\hline & Degrees of Freedom & 16 & 16 & 13 & a \\
\hline & $R^{2}$ & 0.97 & 0.97 & 0.96 & a \\
\hline & Initial $\left(\mathrm{mmol} \mathrm{kg}^{-1}\right.$ embryo $\left.^{-1} \mathrm{~d}^{-1}\right)$ & 0.152 & 0.497 & 3.679 & 26.83 \\
\hline & $\mathrm{X}_{0}(\mathrm{~d})$ & 16 & 18 & 2 & 0.17 \\
\hline \multirow[t]{3}{*}{ Lactate } & Final $\left(\mathrm{mmol} \mathrm{kg}^{-1} \mathrm{embryo}^{-1} \mathrm{~d}^{-1}\right)$ & 0.090 & 0.253 & 2.088 & 3.322 \\
\hline & Degrees of Freedom & 16 & 16 & 13 & 9 \\
\hline & $R^{2}$ & 0.80 & 0.97 & 0.95 & 0.96 \\
\hline
\end{tabular}

a GABA was barely detectable in WS 42 embryos and did not accumulate significantly (Regression analysis, $m=0.124, R^{2}=0.134, P=0.242$ ). 


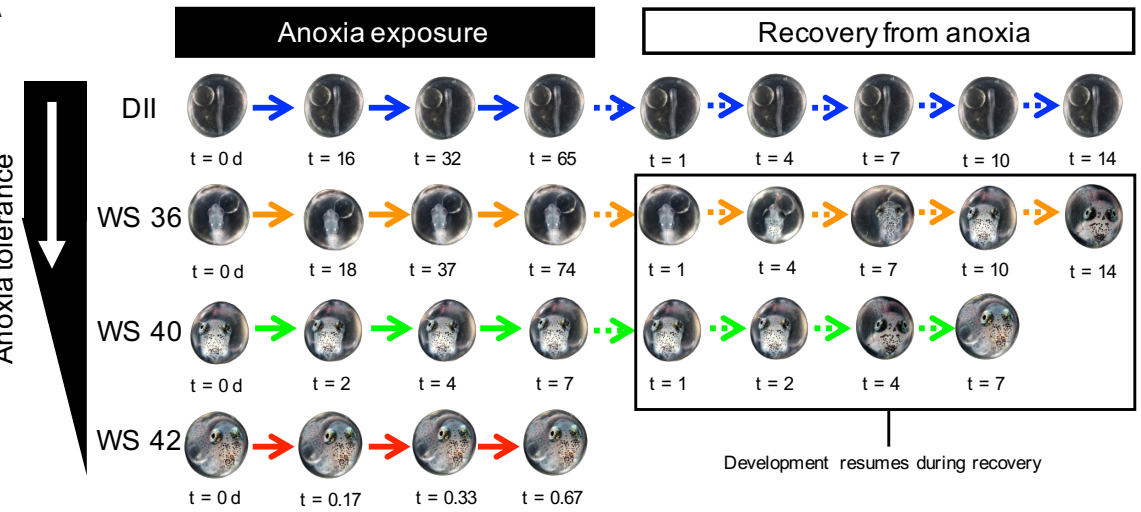

B

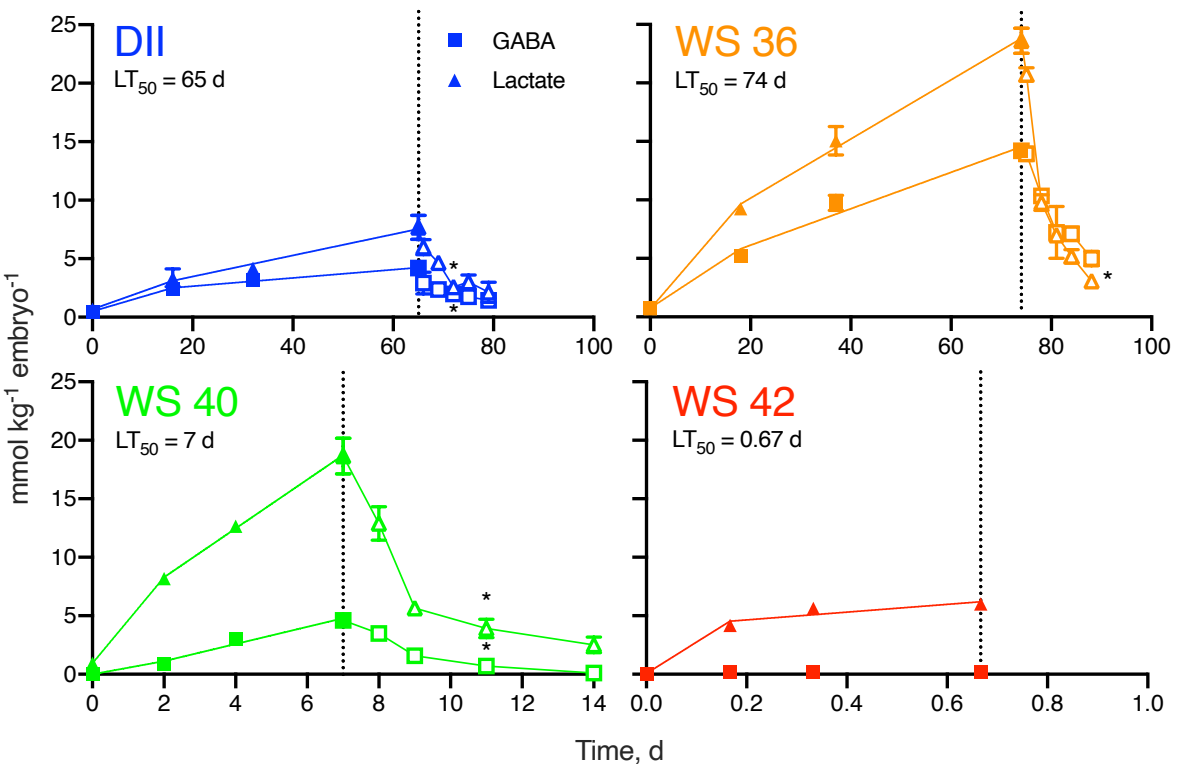

Figure 2.1. GABA and lactate accumulation in embryos of Austrofundulus limnaeus during exposure to anoxia and aerobic recovery from anoxia at $25^{\circ} \mathrm{C}$. (A) A schematic showing the experimental design and sampling regimen used. Embryos from 4 developmental stages were sampled at their lethal time to $50 \%$ mortality $\left(\mathrm{LT}_{50}\right)$ in anoxia, and at the $\mathrm{LT}_{50} / 2$ and $\mathrm{LT}_{50} / 4$ (Meller et al., 2012; Podrabsky et al., 2007; Riggs and Podrabsky, 2017). Following exposure to anoxia, embryos were sampled during aerobic recovery from anoxia for up to $14 \mathrm{~d}$. WS 42 embryos did not recover from anoxia and thus samples were not collected. DII embryos (WS 32/33) remained in diapause during recovery, whereas WS 36 and WS 40 embryos resumed development. Arrow colors match the colors used in subsequent figures for each developmental stage. WS, Wourms' stage; DII, diapause II. (B) Accumulation of lactate and GABA during anoxia and aerobic recovery from anoxia. Vertical dotted lines indicate the LT 50 of each developmental stage. Closed symbols indicate time points in anoxia while open symbols indicate timepoints during aerobic recovery. Each replicate consists of 5 embryos. Symbols represent means \pm SEM $(N=4$ for DII, WS 36 , and WS 40; $N=3$ for WS 42). Analysis of variance (ANOVA, $P<0.0001$ ) indicates a significant accumulation of GABA and lactate for DII, WS 36 and WS 40 embryos, while only lactate accumulated in WS 42 embryos. Asterisks indicate the first timepoints in recovery that are not significantly different from $\mathrm{t}=0$ (Dunnett's post hoc, $P<0.05$ ). Rates of GABA and lactate accumulation were calculated from the slopes of metabolite concentrations during exposure to anoxia. For each developmental stage, initial and final slopes were calculated using segmental linear regression. See Table 2.1 for results of regression analyses. 
A


B
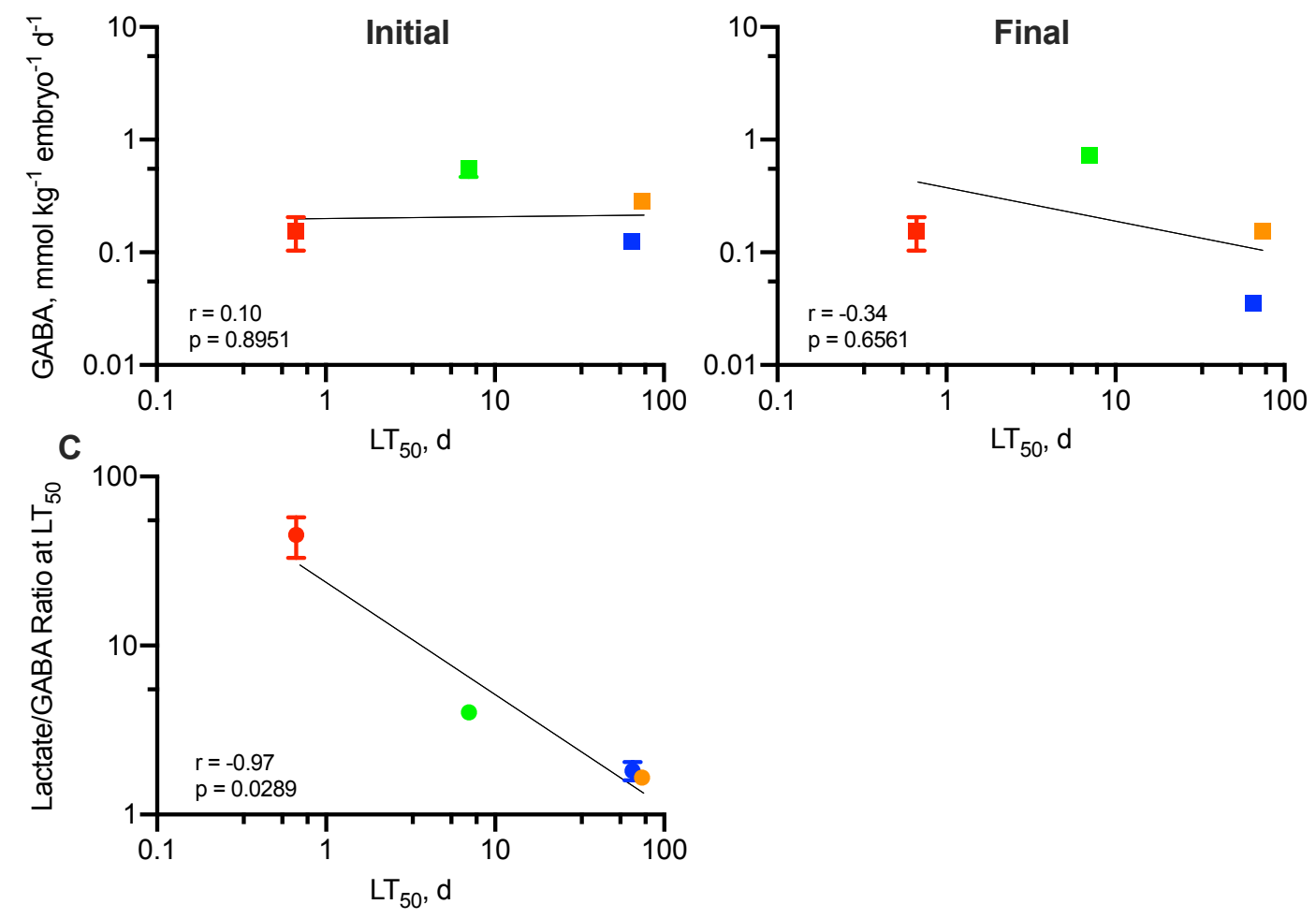

Figure 2.2. The relationship between lethal time to $50 \%$ mortality (LT $\left.{ }_{50}\right)$ and anoxic GABA and lactate accumulation in embryos of $\boldsymbol{A}$. limnaeus. (A) LT 50 was highly correlated with initial rates of lactate accumulation in anoxia in embryos of $A$. limnaeus (Correlation analysis, $P<0.05$ ). When initial rates of lactate accumulation of early developing embryos from Podrabsky et al. (2007) are included, the relationship was strengthened (Correlation analysis, $P<0.0001$ ). Slopes from Podrabsky et al. (2007) and data presented here are similar (ANCOVA, $P=0.1886$ ). Final rates of lactate accumulation were not significantly correlated with survival (Correlation analysis, $P=0.0940)$. Each replicate consists of 5 embryos. Symbols represent means $\pm \operatorname{SEM}(N=4$ for DII, WS 36, and WS 40; $N=3$ for WS 42). Error bars for certain stages are within the symbol. (B) There was no correlation found between $\mathrm{LT}_{50}$ and rates of GABA accumulation (Correlation analysis $P>0.05$ ). (C) There was a negative relationship between $\mathrm{LT}_{50}$ and the ratio of lactate to GABA accumulated during anoxia (Correlation analysis, $P<0.05$ ). Values were determined by dividing the total lactate by total GABA accumulated at each stage's respective $L_{50}$ for each biological replicate. Note that $x$ - and $y$-axes are on log scale for all panels. 


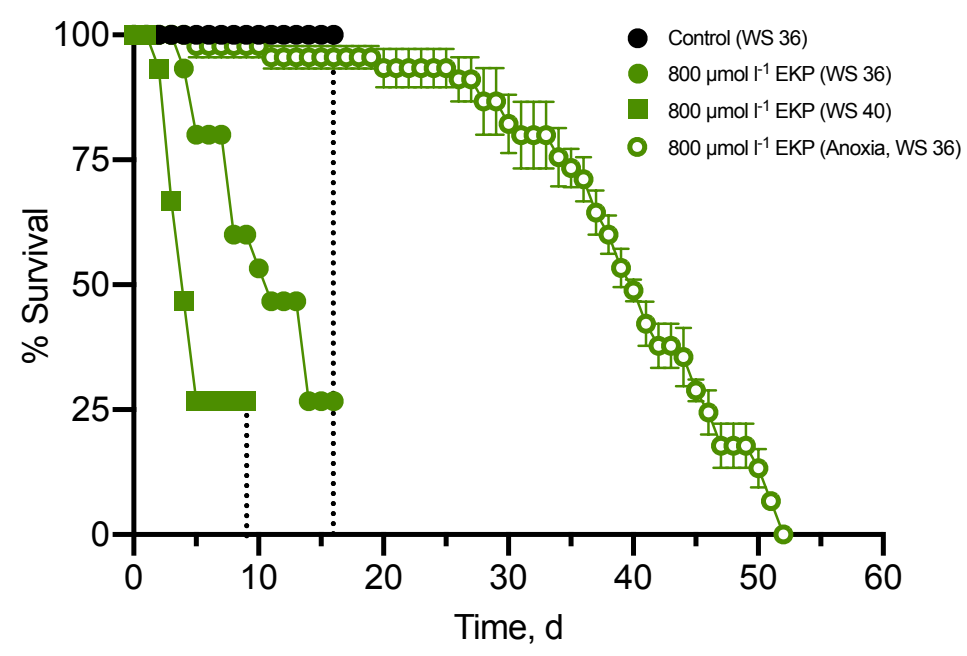

Figure 2.3. Survival of embryos to $800 \mathrm{\mu mol} \mathrm{I}^{-1}$ ethyl ketopentenoate (EKP) in normoxia and anoxia. Wourms' stage (WS) 36 embryos exposed to EKP in anoxia survived longer than developing embryos (WS 36 and WS 40) exposed to EKP in normoxia. WS 40 embryos exhibited mortality after $2 \mathrm{~d}$ of exposure, whereas WS 36 embryos exhibited mortality after $4 \mathrm{~d}$. Vertical dotted lines indicate completion of embryological development. WS 36 embryos in anoxia remained at WS 36 for the duration of EKP exposure. Filled symbols represent percent survival of 15 individually monitored embryos in normoxia. Open symbols represent mean survival \pm SEM of groups of 15 embryos $(N=3)$ and are from Figure 2.4 and are provided here for ease of comparison. 
A
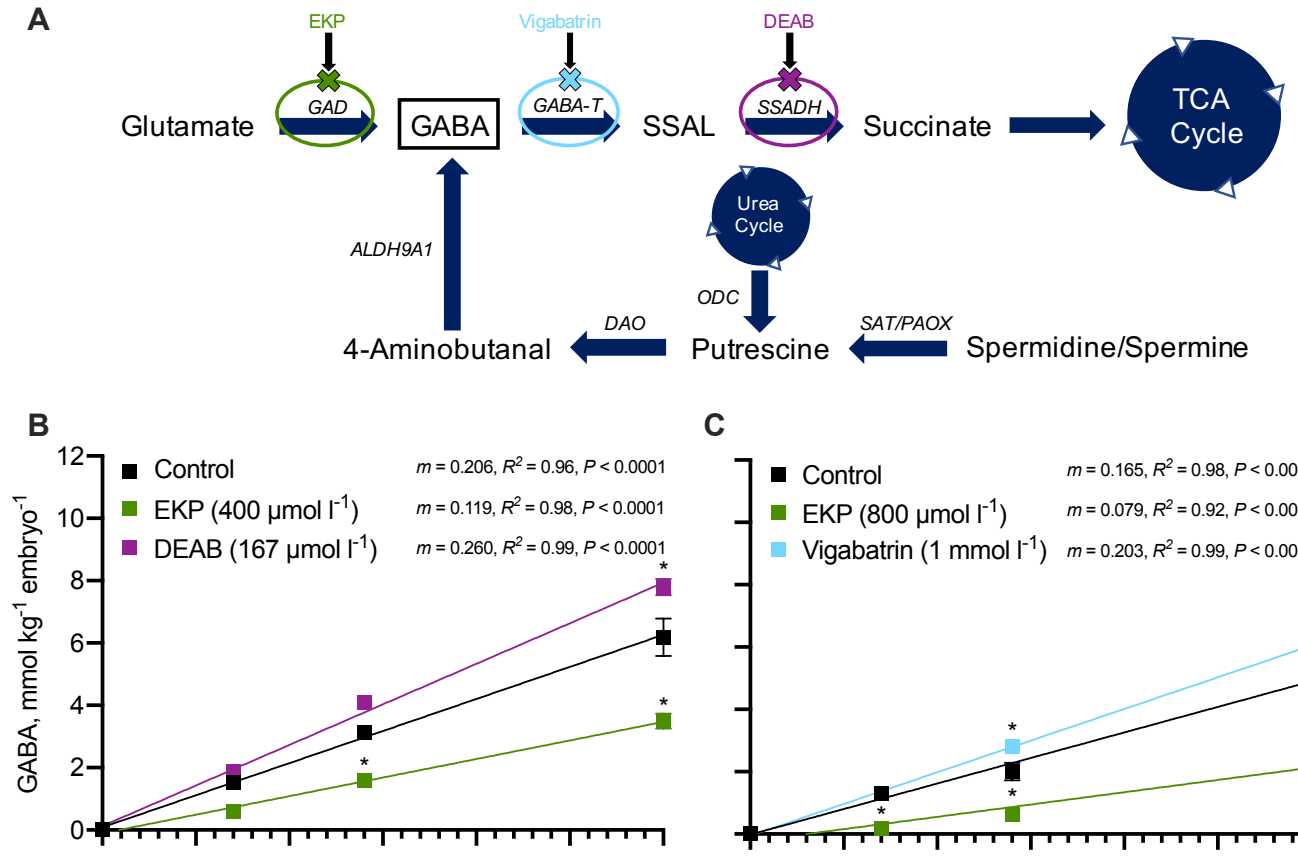

C
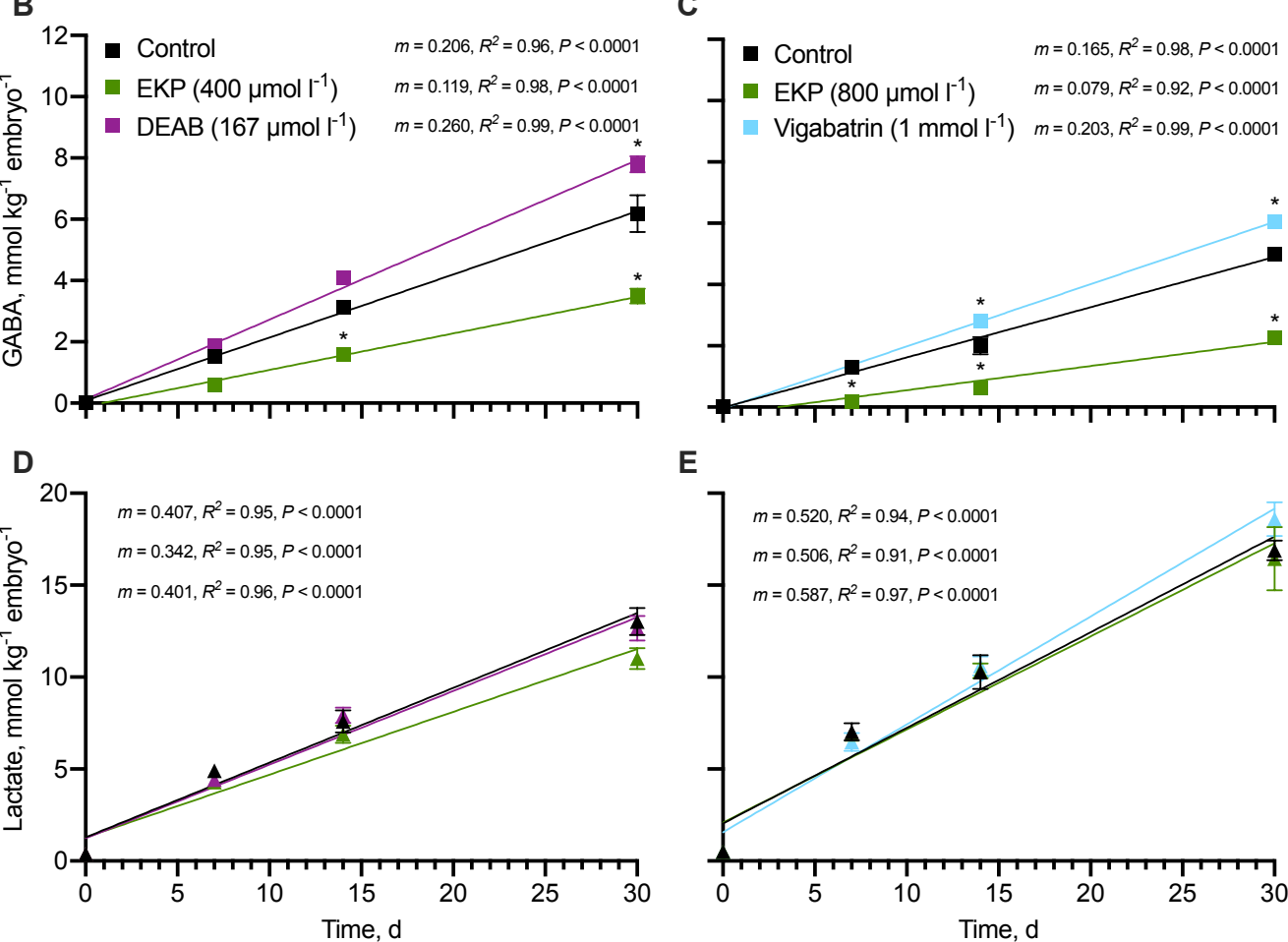

E

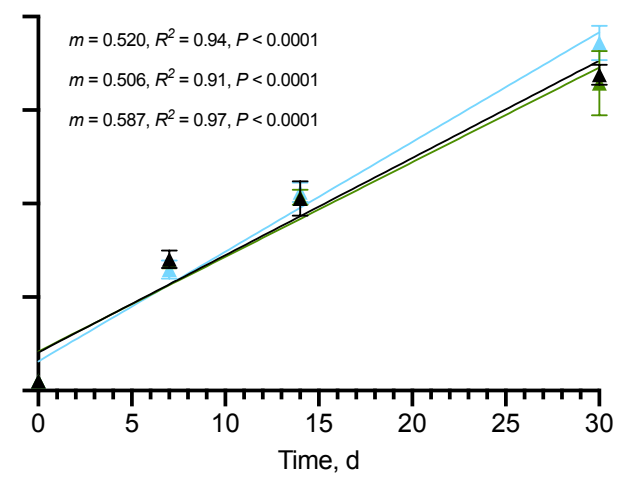

Figure 2.4. GABA and lactate accumulation during inhibition of the GABA synthesis and degradation pathway in Wourms' stage 36 embryos exposed to anoxia. (A) A schematic depicting canonical and non-canonical synthesis and metabolic degradation of GABA and the key enzymes pharmacologically inhibited in experiments. GAD, glutamate decarboxylase; GABA-T, GABA transaminase; SSAL, succinic semialdehyde; SSADH, succinic acid semialdehyde dehydrogenase; EKP, ethyl ketopentenoate; DEAB, N,N-diethylaminobenzaldehyde; ALDH9A1, aldehyde dehydrogenase; DAO, diamine oxidase; ODC, ornithine decarboxylase; SAT, spermidine/spermine N1-acetyltransferase; PAOX, polyamine oxidase. (B,C) GABA accumulated in a linear manner for the control, EKP, DEAB, and vigabatrin treatments (Regression analysis, $P$ $<0.0001)$. Rates of GABA accumulation $(m)$ for all treatments and the controls were significantly different from one another (B: ANCOVA, $F=55.96, P<0.0001$; C: ANCOVA, $F=74.54, P<$ $0.0001)$. Asterisks indicate treatment timepoints that were significantly different from the control (two-way ANOVA with Tukey's HSD, $P<0.05)$. Symbols represent means \pm SEM $(N=3)$ and each replicate contained 5 embryos. (D,E) Lactate accumulated in a linear manner for the control, EKP, DEAB, and vigabatrin treatments (Regression analysis, $P<0.0001$ ). Rates of lactate accumulation $(m)$ were similar for the controls and all treatments (D: ANCOVA, $F=1.750, P=$ 0.19; E: ANCOVA, $F=1.043, P=0.36$ ). No timepoints for embryos treated with EKP, DEAB, or vigabatrin were significantly different from one another or the control (two-way ANOVA with Tukey's HSD, $P>0.05)$. 

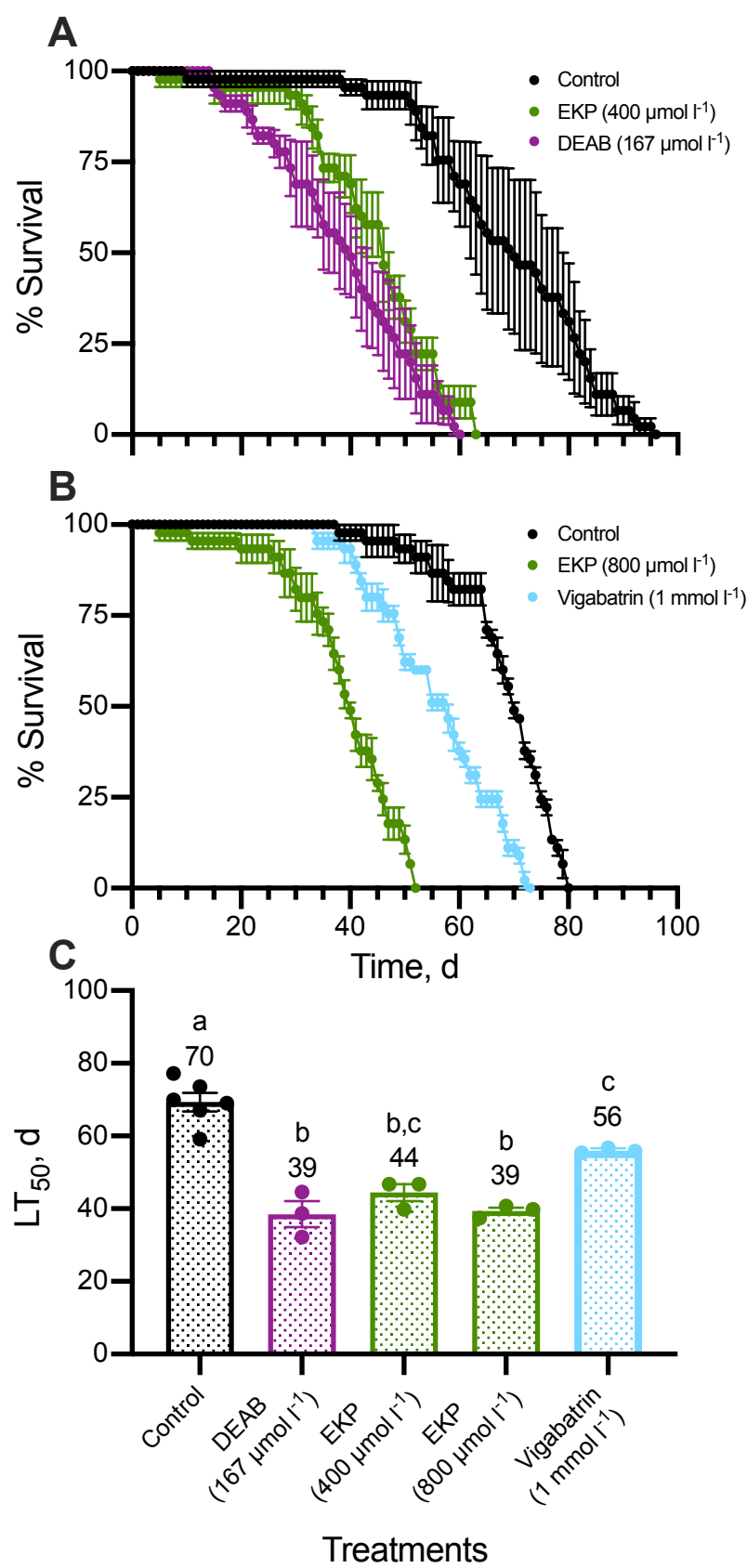

Figure 2.5. Survival of Wourms' stage 36 embryos continuously exposed to GAD inhibitor EKP, SSADH inhibitor DEAB, and GABA-T inhibitor vigabatrin in anoxia. Groups of 15 embryos were exposed to embryo medium containing (A) $400 \mu \mathrm{mol} \mathrm{I}^{-1} \mathrm{EKP}$ or $167 \mu \mathrm{mol} \mathrm{l}^{-1} \mathrm{DEAB}$, (B) $800 \mu \mathrm{mol} \mathrm{I}^{-1} \mathrm{EKP}$, or $1 \mathrm{mmol} \mathrm{I}^{-1}$ vigabatrin in anoxia at $25^{\circ} \mathrm{C}$. Symbols are means $\pm \mathrm{SEM}(\mathrm{N}=$ 3). (C) Lethal time to $50 \%$ mortality $\left(\mathrm{LT}_{50}\right)$ for embryos exposed to anoxia. Different letters indicate significant differences in $\mathrm{LT}_{50}$ between treatments (one-way ANOVA with Tukey's HSD test, $P<0.05$ ). Bars are means \pm SEM (Control, $N=6$; Inhibitors, $N=3$ ) and symbols represent individual replicates. GAD, glutamate decarboxylase; EKP, ethyl ketopentenoate; SSADH, succinic acid semialdehyde dehydrogenase; DEAB, N,N-diethylaminobenzaldehyde; GABA-T, GABA transaminase. 


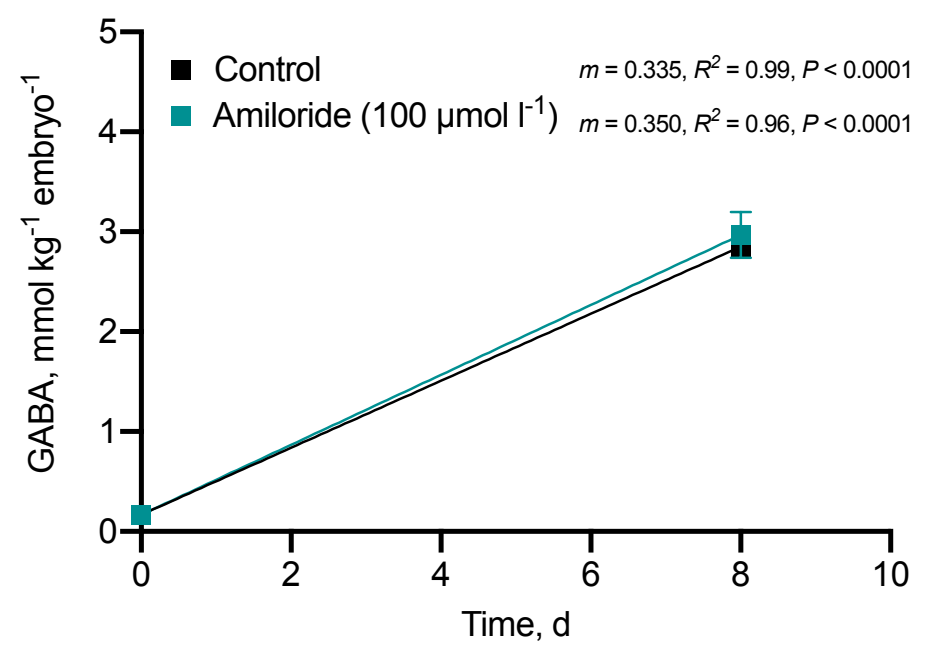

Figure 2.6. GABA accumulation during inhibition of diamine oxidase (DAO) by amiloride in Wourms' stage 36 embryos exposed to anoxia. Rates of GABA accumulation $(m)$ were similar for the control and amiloride treatments (ANCOVA, $F=0.2208, P=0.65$ ). There was no significant difference in GABA accumulated after $8 \mathrm{~d}$ (unpaired t-test, $P=0.64$ ). Symbols represent means \pm SEM $(N=4)$ and each replicate contained 5 embryos. Slopes $(m)$ were calculated using linear regression analysis. 


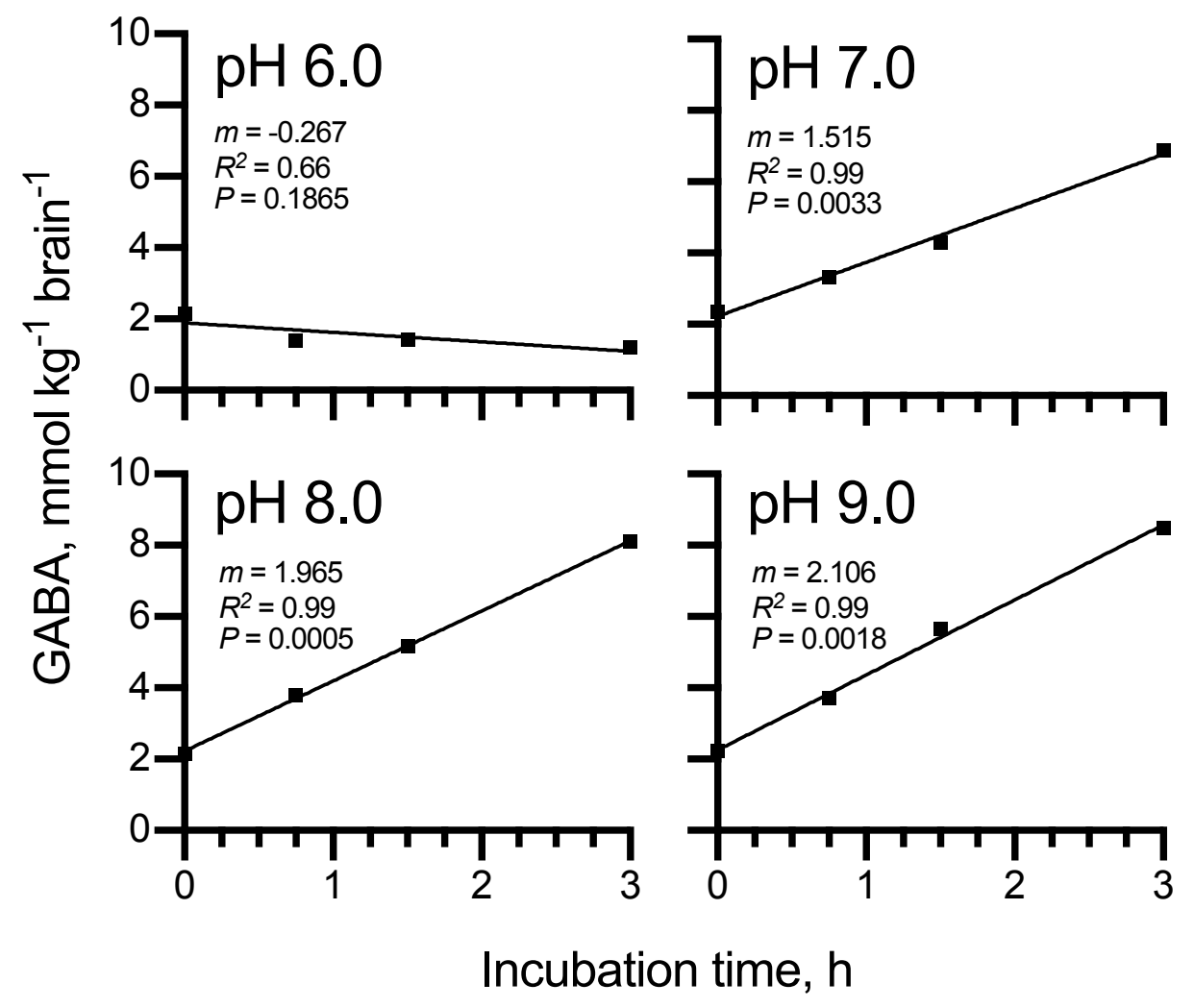

Figure 2.7. Glutamate decarboxylase (GAD) activity in adult male $A$. limnaeus brain homogenates in normoxia at $25^{\circ} \mathrm{C}$. Adult brain homogenates generate GABA that are significantly greater than 0 at $\mathrm{pH} 7.0,8.0$, and 9.0 (Regression analysis, $P<0.005$ ), while pH 6.0 homogenates did not show a significant accumulation of GABA after $3 \mathrm{~h}$ of incubation (Regression analysis, $P=0.1865$ ). Rates of GABA production and thus GAD activity were based on the slope $(m)$ of GABA accumulation over time. Symbols are individual replicates. All replicates between $\mathrm{pH}$ treatments originated from the same brain homogenate. 

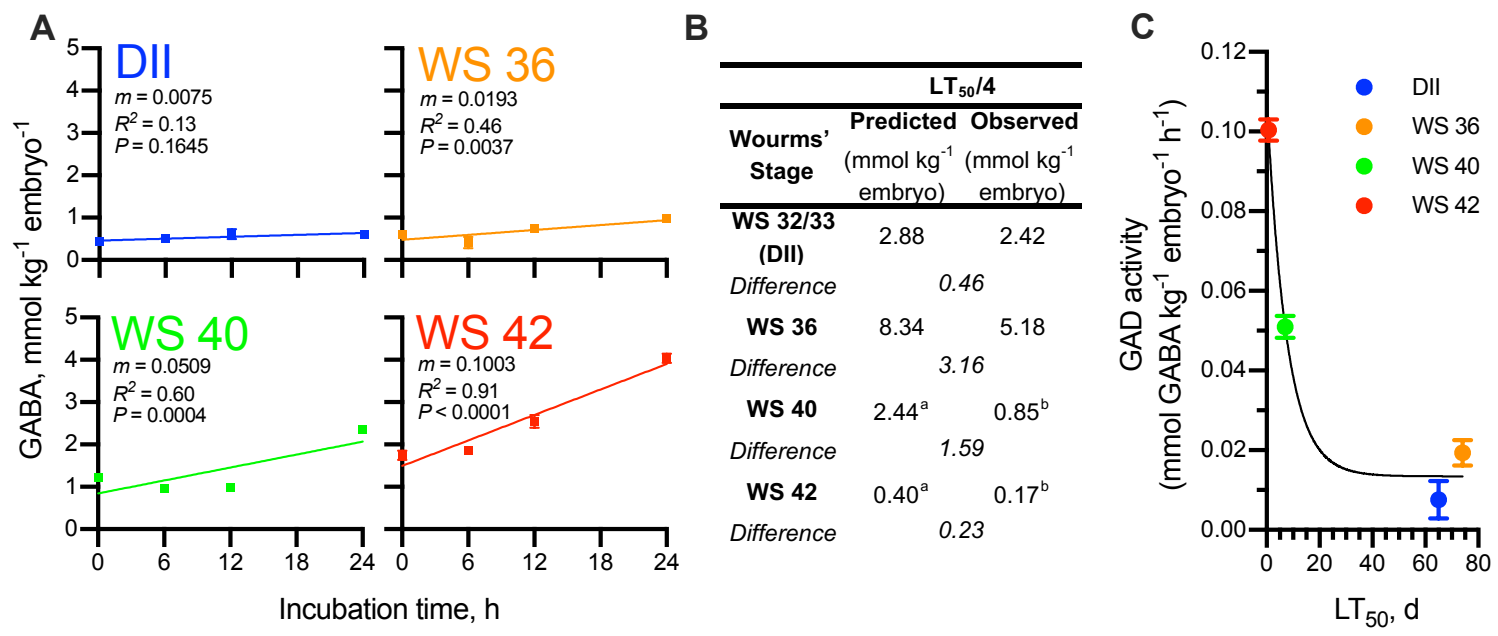

Figure 2.8. Glutamate decarboxylase (GAD) activity in A. limnaeus embryo homogenates across development in normoxia at $25^{\circ} \mathrm{C}$. (A) Post-DIl embryo homogenates generate GABA at low rates that are significantly greater than 0 (Regression analysis, $P<0.01$ ) while DIl embryo homogenates did not show a significant accumulation of GABA after $24 \mathrm{~h}$ of incubation (Regression analysis, $P=0.1701$ ). Rates of GABA production and thus GAD activity were based on the slope $(m)$ of GABA accumulation over time. Symbols are means \pm SEM $(N=4)$. Each replicate consisted of 13 embryos. (B) Predicted initial GABA accumulation based on GAD activity compared to observed GABA accumulated in embryos exposed to anoxia (from Fig 2.1B). Despite not accumulating significant amounts of GABA during anoxia, observed mean GABA levels are included for WS 42 embryos. Different letters indicate significant differences in predicted and observed values within developmental stage (unpaired t-test, $P<0.05$ ). (C) There is a negative relationship between $L T_{50}$ and GAD activity in embryos of $A$. limnaeus. Exponential one-phase decay analysis provides a good fit for the two variables $\left(R^{2}=0.96\right)$. Symbols represent means \pm SEM $(N=4)$. 

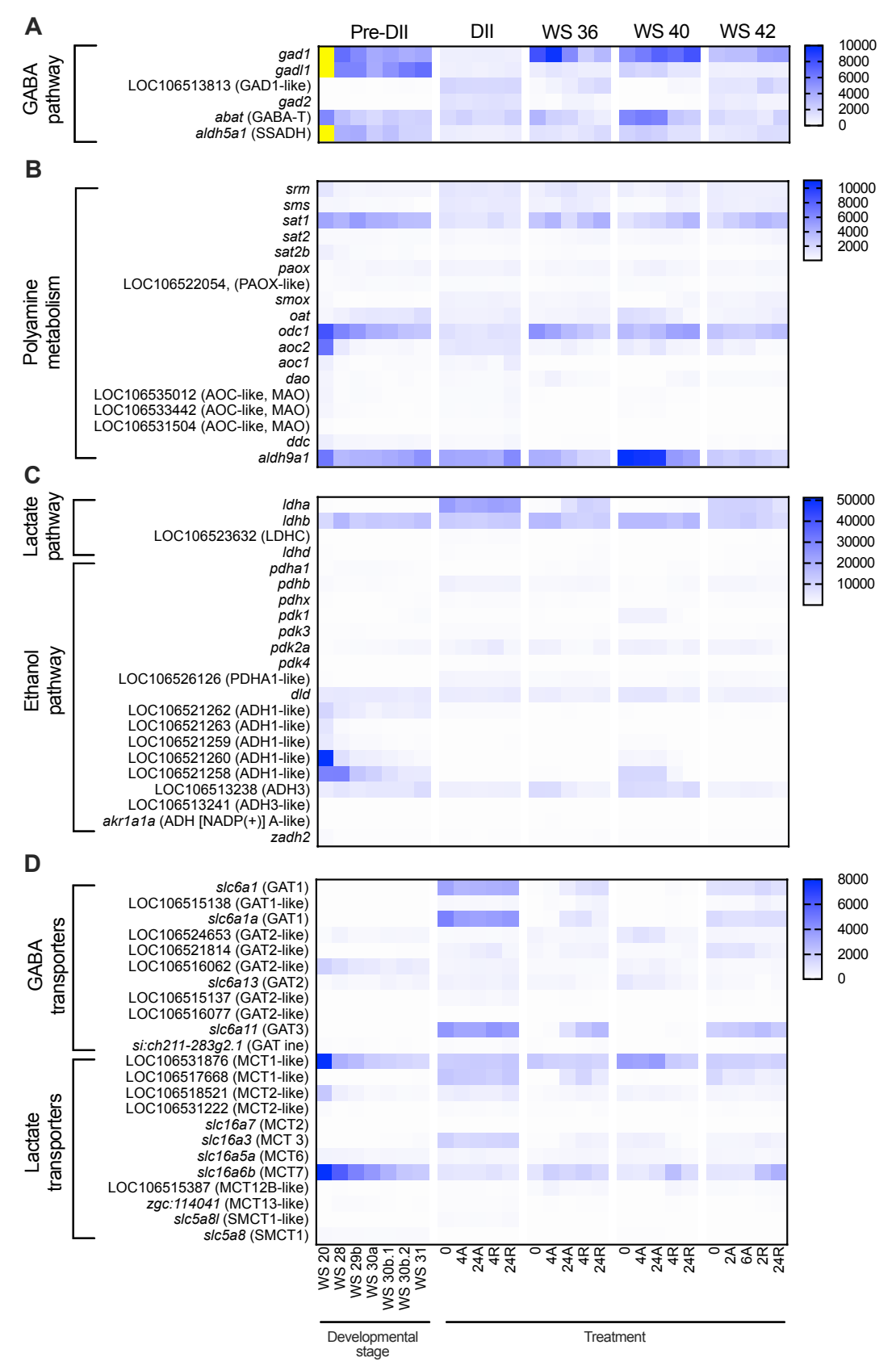

Figure 2.9. Transcript levels for GABA, polyamine, lactate, and ethanol pathways in $A$.

limnaeus as determined by RNA sequencing. Enzyme systems are organized into groups: (A) GABA synthesis and degradation, (B) polyamine metabolism, (C) lactate and ethanol synthesis, and (D) GABA and lactate transportation. Data for pre-Dll development (WS 20-31) were obtained from embryos incubated at $20^{\circ} \mathrm{C}$ and were collected at the developmental stages shown at the bottom of the heatmap. Embryos in DII and post-DII were incubated at $25^{\circ} \mathrm{C}$ and timepoints for anoxia and aerobic recovery are shown below the heatmap: 0, normoxia; $4 \mathrm{~A}, 4 \mathrm{~h}$ anoxia; $24 \mathrm{~A}$, $24 \mathrm{~h}$ anoxia; $4 \mathrm{R}, 4 \mathrm{~h}$ recovery; $24 \mathrm{R}, 24 \mathrm{~h}$ recovery; $2 \mathrm{~A}, 2 \mathrm{~h}$ anoxia; $2 \mathrm{R}, 2 \mathrm{~h}$ recovery. Expression levels are reported as mean fragments per kilobase per million mapped reads (FPKM). $N=3$ for pre-DIl stages; $N=4$ for DII, WS 36, and WS $42 ; N=6$ for WS 40 . WS, Wourms' stage. Cells in yellow indicate transcript levels that were overexpressed (FPKM $=11,000-30,000)$. 


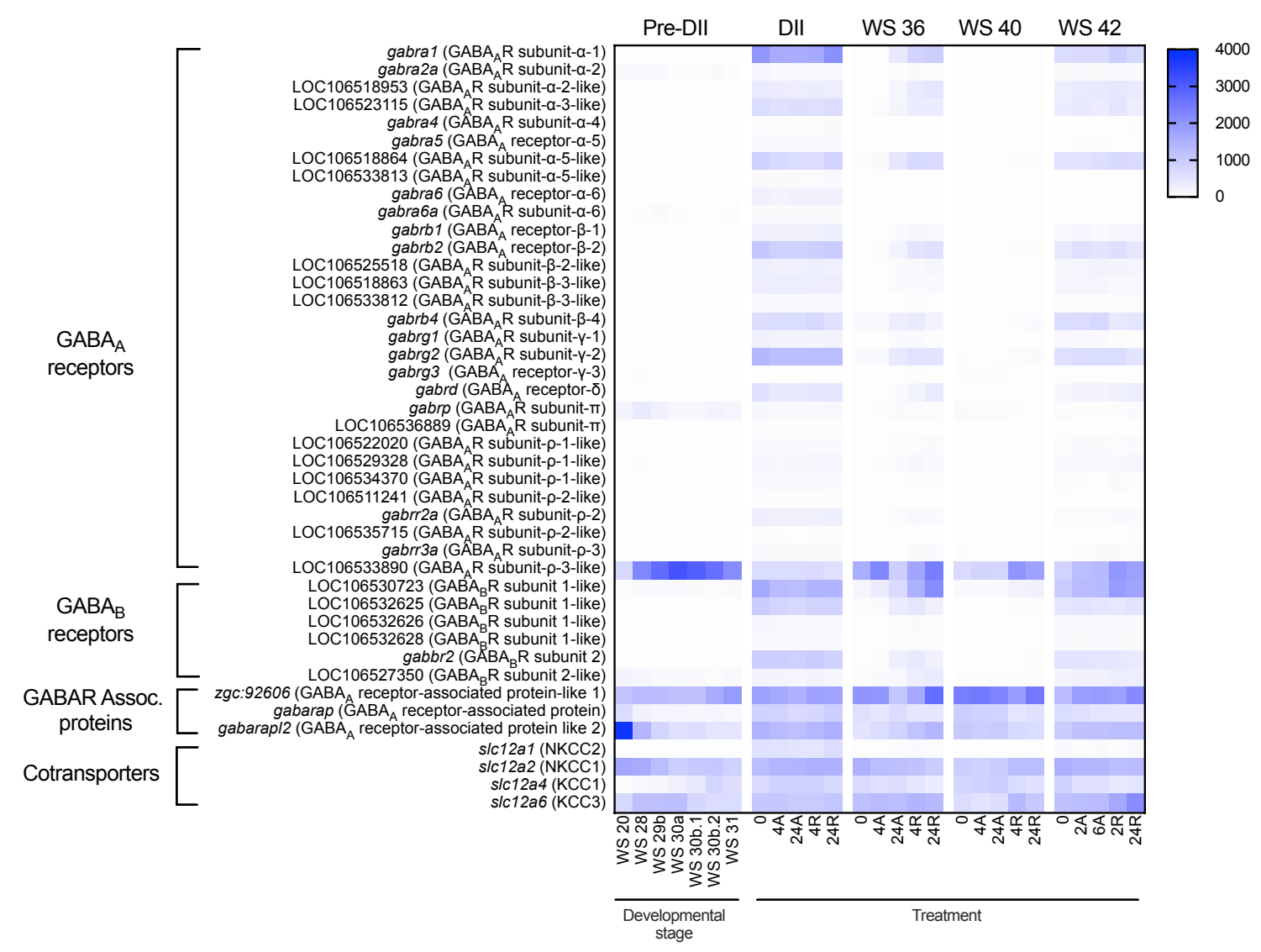

Figure 2.10. Transcript levels for GABA signaling and cotransporters in $A$. limnaeus as determined by RNA sequencing. Enzyme systems are organized into groups: GABA $A_{A}$ receptors and subunits, GABA receptors and subunits, GABA receptor associated proteins, and $\mathrm{Na}^{+}-\mathrm{K}^{+}-\mathrm{Cl}^{-}$ (NKCC) and $\mathrm{K}^{+}-\mathrm{Cl}^{-}$-cotransporters (KCC). Data for pre-DIl development (WS 20-31) were obtained from embryos incubated at $20^{\circ} \mathrm{C}$ and were collected at the developmental stages shown at the bottom of the heatmap. Embryos in DII and post-DII were incubated at $25^{\circ} \mathrm{C}$ and timepoints for anoxia and aerobic recovery are shown below the heatmap: 0, normoxia; $4 \mathrm{~A}, 4 \mathrm{~h}$ anoxia; $24 \mathrm{~A}$, $24 \mathrm{~h}$ anoxia; 4R, $4 \mathrm{~h}$ recovery; 24R, $24 \mathrm{~h}$ recovery; $2 \mathrm{~A}, 2 \mathrm{~h}$ anoxia; $2 \mathrm{R}, 2 \mathrm{~h}$ recovery. Expression levels are reported as mean fragments per kilobase per million mapped reads (FPKM). $N=3$ for pre-DII stages; $N=4$ for DII, WS 36 , and WS $42 ; N=6$ for WS 40 . WS, Wourms' stage. 

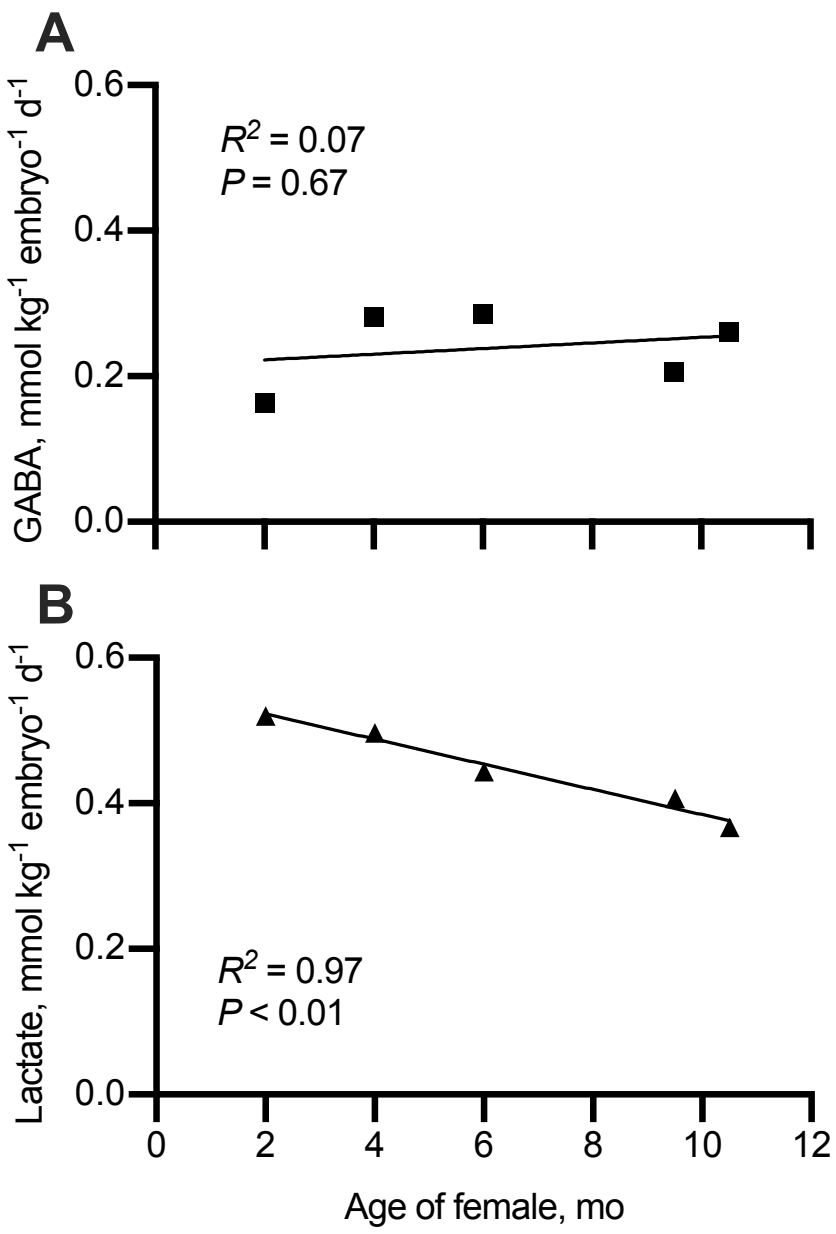

Figure 2.11. The relationship between female age and rates of GABA and lactate accumulation in anoxic Wourms' stage 36 embryos. (A) The rate of GABA accumulation in embryos did not correlate with female age; however (B) there was a significant negative linear relationship in the rate of lactate accumulation with female age. Each symbol represents a single rate of accumulation calculated from biological replicates $(N=3-4)$ sampled up to $30 \mathrm{~d}$ in anoxia. 


\section{Chapter 3}

\section{No water, no problem: Stage-specific metabolic responses to dehydration stress in annual killifish embryos}

This chapter has previously been submitted for publication:

Zajic D, Nicholson J, and Podrabsky J. No water, no problem: Stage-specific metabolic responses to dehydration stress in annual killifish embryos. J Exp Biol Submitted: 2020.

\section{Introduction}

Water is essential for life and the threat of dehydration due to evaporative water loss is ever-present in terrestrial organisms and especially in aquatic organisms that exploit terrestrial environments (Martin and Cooper, 1972; Martin and Carter, 2013; Martin et al., 2004; Martin, 1999; Moravek and Martin, 2011). In all terrestrial organisms, respiratory gas exchange and water loss are closely related: if oxygen and carbon dioxide can be exchanged, so can water vapor (Danks, 2000). Subsequently, nearly all terrestrial animals have evolved mechanisms to dramatically reduce loss of water through the integument and support efficient gas exchange through specialized respiratory organs. Those animals that do rely on cutaneous gas exchange must remain in moist or semiaquatic habitats (e.g., salamanders and frogs). Even fewer aquatic organisms can exploit terrestrial habitats for prolonged periods of time. Annual killifishes exploit ephemeral pond habitats that can be devoid of liquid water for several 
months and perhaps even years (Polačik and Podrabsky, 2015). Survival of prolonged water stress is invested solely in drought-tolerant embryos that survive in the soil of dry ponds. Despite the clear importance of dehydration tolerance to the survival of annual killifishes, many questions remain regarding how long embryos can survive without water, their metabolic rate during aerial exposure, and the molecular mechanisms that support survival.

Many fish embryos that experience aerial conditions are found in moist environments, therefore evading severe desiccation (Martin, 1999; McDowall and Charteris, 2006; Podrabsky et al., 2010b; Taylor, 1999; Tingaud-Sequeira et al., 2009; Wells et al., 2015). The duration spent in the aerial environment for many species is usually short, on the magnitude of days or weeks (Martin et al., 2011; Thompson et al., 2017). The annual killifishes are unique exceptions in that they regularly endure long periods (months) of no water. The annual killifish, Austrofundulus limnaeus, is an emerging model for developmental physiology and ecology because of their wide array of environmental stress tolerances, annotated genome, and the ability to manipulate developmental trajectories in the lab (Podrabsky et al., 2017; Podrabsky et al., 2010a; Romney et al., 2018; Wagner et al., 2018). Adult $A$. limnaeus live in ephemeral ponds in the coastal deserts of Venezuela and their embryos have the remarkable ability to tolerate anoxic and dehydrating conditions for months (Podrabsky et al., 2016). Survival is attributed to the ability of these embryos to enter a state of profound metabolic dormancy (diapause) as a part of their normal development (Podrabsky and Hand, 1999; Podrabsky and Hand, 2000). Embryos can enter diapause at three 
distinct stages termed diapause I, II, and III (Podrabsky et al., 2017; Wourms, 1972b; Wourms, 1972c). In A. limnaeus, diapause II (DII) is the most stresstolerant stage and is likely responsible for long-term survival of dehydrating conditions (Podrabsky et al., 2016; Podrabsky et al., 2001; Polačik et al., 2020).

Embryos of $A$. limnaeus lose water in the first few days of aerial exposure, but after about a week the rate of water loss approaches zero (Podrabsky et al., 2001). Importantly, most of this water is lost from the extraembryonic perivitelline compartment, while the embryo appears to remain fully hydrated. Dramatically reduced permeability to water vapor suggests highly limited gas exchange in general, and thus we hypothesize that this mechanism for survival of dehydration stress may result in lower respiratory gas exchange and produce a self-imposed hypoxia or anoxia. Lack of oxygen (anoxia) is known to induce quiescence (halting development) in developing embryos of $A$. limnaeus (Podrabsky et al., 2007), and thus we also predict that dehydration may directly or indirectly induce developmental arrest.

Here we report on the survival and metabolic responses of dormant (DII) and actively developing post-diapause II embryos under aerial conditions to evaluate how long these embryos might survive water stress. We evaluate these data in relation to the ecology of embryonic development in annual killifishes.

These data support a stage-specific response to dehydration stress that leads to increased variation in developmental rate and presumably physiological phenotypes in late-stage embryos only after they exit diapause II. Thus, longterm survival in the soil may be supported by entrance into diapause II while 
intrinsic variation in developmental rate in response to a mixture of dehydration and oxygen stress may lead to a variety of developmental outcomes.

\section{Materials and Methods}

\section{Animal husbandry and embryo collection}

Adult annual killifish were housed and embryos collected as previously described (Podrabsky, 1999). This work was performed under established protocols which were approved and reviewed by the Portland State University Institutional Animal Care and Use Committee (PSU IACUC protocols \#33 and 64). Briefly, adult annual killifish were kept in male-female pairs and spawned semiweekly. Embryos were collected and stored at $25^{\circ} \mathrm{C}$ with no light in $15 \times 100$ $\mathrm{mm}$ plastic Petri dishes in media that resembles the environmental conditions from which adults were collected in $1995\left(10 \mathrm{mmol} \mathrm{I}^{-1} \mathrm{NaCl}, 2.15 \mathrm{mmol} \mathrm{I}^{-1} \mathrm{MgCl}_{2}\right.$, $0.8 \mathrm{mmol} \mathrm{I}^{-1} \mathrm{CaCl}_{2}, 0.14 \mathrm{mmol} \mathrm{I}^{-1} \mathrm{KCl}, 1.3 \mathrm{mmol} \mathrm{I}^{-1} \mathrm{MgSO}_{4}$ ) (Podrabsky, 1999; Podrabsky et al., 1998). During the first 4 days post-fertilization (dpf), embryo medium contained methylene blue $(0.0001 \%)$ to prevent fungal infection.

Embryos were then treated with two 5 min washes of a solution of $0.01 \%$ sodium hypochlorite (separated by a 5 min rest in embryo medium) to prevent bacterial and fungal growth, as previously described (Podrabsky, 1999). Following sodium hypochlorite treatment, embryos were transferred to embryo medium containing $10 \mathrm{mg} \mathrm{l}^{-1}$ gentamicin sulfate and allowed to develop to DII (32-46 d). To break DII, embryos were subjected to a temperature of $30^{\circ} \mathrm{C}$ and full spectrum light for $48 \mathrm{~h}$ (Meller et al., 2012). Following this treatment, embryos were sorted into 
synchronized cohorts of embryos by developmental stage (Podrabsky et al., 2017).

\section{Embryonic stages investigated}

Experiments were performed on four developmental stages of embryos (Podrabsky et al., 2017; Wourms, 1972b; Wourms, 1972c) to capture a gradient of stress tolerance levels and physiology: Wourms' stage (WS) 32/33 or diapause II (DII), WS 36 (4 days post-diapause II (dpd)), WS 40 (12 dpd), and WS 42 (20 dpd). DII embryos are metabolically dormant and have halted development, whereas the other stages are metabolically active and developing.

\section{Tolerance of aerial exposure}

Embryos (DII, WS 36, WS 40, WS 42) were exposed to $85 \%$ relative humidity $(\mathrm{RH})$ air at $25^{\circ} \mathrm{C}$ with no light in a sealed plastic desiccator (Nalgene, $250 \mathrm{~mm}$ diameter). Relative humidity was controlled by using a saturated solution of potassium chloride $(750 \mathrm{ml})$ placed below the shelf and continually mixed with a stir bar to ensure uniform $\mathrm{RH}$ within the aerial portion of the chamber (Podrabsky et al., 2001; Winston and Bates, 1960). Prior to dehydration exposure, embryos were treated with sodium hypochlorite (see above) and were allowed to recover for $2 \mathrm{~h}$ at $25^{\circ} \mathrm{C}$ without light. Groups of 20 embryos $(N=3)$ per stage were removed from their aqueous medium and placed in Petri dishes (50 mm, PDF2047S0, Fisher Scientific, Hampton, NH, USA) containing a sterilized filter pad saturated with $2.5 \mathrm{ml}$ of embryo medium containing $10 \mathrm{mg} \mathrm{l}^{-1}$ gentamicin 
sulfate (Podrabsky et al., 2001). Embryos were monitored until $100 \%$ mortality was reached. Embryos were determined dead when they lost their shape and shine. Survival between stages was compared by calculating lethal time to $50 \%$ mortality $\left(L T_{50}\right)$ in response to aerial exposure.

\section{Metabolic rate (respirometry)}

Aerial incubation

Diapause II. Embryos were treated with sodium hypochlorite (see above) and incubated in embryo medium containing $10 \mathrm{mg} \mathrm{l}^{-1}$ gentamicin sulfate and 2.5 $\mu \mathrm{g} \mathrm{I}^{-1}$ amphotericin B (10128-872, VWR, Radnor, PA, USA) for $24 \mathrm{~h}$ prior to aerial exposure. For aerial incubation, DII embryos were placed on filter pads containing $2.5 \mathrm{ml}$ embryo medium containing gentamicin and amphotericin B using sterile technique within a biosafety cabinet. Care was taken to make certain that single embryos were isolated and not touching other embryos. DII embryos were then exposed to $85 \% \mathrm{RH}$ at $25^{\circ} \mathrm{C}$ without light for $11 \mathrm{~d}$ to allow equilibration of the filter pad with the aerial environment. Four biological replicates $(N=4)$, comprised of five embryos each, were placed in standard opening $2 \mathrm{ml}$ borosilicate glass crimp-top vials (03-391-2, 12 OD x $32 \mathrm{~mm}$ long, Fisher Scientific, Hampton, NH, USA) containing a 4 mm RedEye oxygen sensor patch for non-invasive oxygen monitoring (Ocean Insight, Largo, FL, USA). Transfer of embryos was done within a biosafety cabinet using sterile technique. Vials containing embryos and sensor patches were crimped with aluminum crimp seals and a black Viton ${ }^{\mathrm{TM}}$ elastomer septa (03-378-343, Thermo Scientific, 
Waltham, MA, USA). Vials and septa were equilibrated at $85 \% \mathrm{RH}$ for $30 \mathrm{~min}$ prior to use. To ensure sterilization of vials and sensor patches, vials containing patches were prepared in a biosafety cabinet and UV-sterilized for several hours prior to addition of embryos.

Wourms' stage 36 embryos. Diapause II embryos were treated with sodium hypochlorite and incubated in embryo medium containing $10 \mathrm{mg} \mathrm{l}^{-1}$ gentamicin sulfate and $2.5 \mathrm{\mu g} \mathrm{l}^{-1}$ amphotericin B for $24 \mathrm{~h}$. Embryos were placed on filter pads containing $2.5 \mathrm{ml}$ embryo medium with gentamicin and amphotericin B using sterile technique and placed into a desiccator at $85 \% \mathrm{RH}$ (see details above). Diapause II was broken under aerial incubation by placing the desiccator under full spectrum light in an incubator at $30^{\circ} \mathrm{C}$. After $72 \mathrm{~h}$, temperature was reduced to $25^{\circ} \mathrm{C}$ and the light was turned off. Following $24 \mathrm{~h}$ of $25^{\circ} \mathrm{C}$ without light, embryos were removed and staged. Filter pads were then weighed to ensure equilibration with chamber relative humidity and showed no difference with the filter pads used for DII treatments. For WS 36 embryos, 7 biological replicates $(N=7)$, comprised of five embryos each were used. Embryos were added to $2 \mathrm{ml}$ vials containing sensor patches and crimp sealed under sterile conditions as described above.

\section{Aqueous incubation}

Diapause II and WS 36 embryos were sorted and treated with sodium hypochlorite as described above. Following treatment, embryos were allowed to recover for $2 \mathrm{~h}$ prior to the start of the respirometry experiment. Embryo medium 
containing $10 \mathrm{mg} \mathrm{l}^{-1}$ gentamicin sulfate was aerated by gentle bubbling with atmospheric air at $25^{\circ} \mathrm{C}$ for $1 \mathrm{~h}$ to ensure dissolved oxygen (DO) saturation. To prevent supersaturation, medium was allowed to equilibrate for 5 min without aeration prior to use. Embryo medium was then added to vials containing 5 embryos each (DII, $N=4$; WS $36, N=5$ ) and a sensor patch. Vials were immediately crimp sealed with Viton ${ }^{\mathrm{TM}}$ caps making sure to leave no air bubbles trapped within the vials.

\section{Oxygen measurement and embryo observation}

Measurements of sensor patch fluorescence were made through the vial wall using a fiber optic probe in conjunction with a NeoFox Oxygen Sensing System (Ocean Insight, Largo, FL, USA). For aqueous conditions, the system was calibrated to $100 \%$ DO saturation using vials containing embryo medium aerated with atmospheric air at $25^{\circ} \mathrm{C}$ and $0 \%$ DO saturation using embryo medium bubbled with $\mathrm{N}_{2}$ for 30 min and equilibrated overnight in a Bactron III anaerobic chamber (Sheldon Manufacturing, Cornelius, OR, USA), containing $5 \% \mathrm{CO}_{2}, 5 \% \mathrm{H}_{2}$, and $90 \% \mathrm{~N}_{2}$. For aerial conditions, the system was calibrated to $100 \%$ saturation using vials containing atmospheric air at $25^{\circ} \mathrm{C}$ and $0 \%$ saturation using $5 \% \mathrm{CO}_{2}, 5 \% \mathrm{H}_{2}$, and $90 \% \mathrm{~N}_{2}$ from the anaerobic chamber. Previous observations illustrated no effects of the anaerobic gas mixture on fluorescence measurements (data not shown). Vials sealed at $0 \%$ and $100 \%$ saturation (both aerial and aqueous) were regularly measured alongside samples. These control vials remained consistent across the entire duration of 
the experiments, and were used as blank vials to control for the possibility of contamination and to ensure stable calibration of the NeoFox Oxygen Sensing System. Oxygen concentration was monitored regularly and rate of oxygen consumption was used as a proxy for metabolic rate. All measurements were taken at $25^{\circ} \mathrm{C}$ with minimal light. Following measurement of oxygen concentration, embryos were observed under a dissection microscope (Leica, S8 APO, Wetzlar, Germany) to record health and developmental stage. Rate of oxygen consumption was calculated using the slope of decrease in oxygen content inside each vial over time divided by the number of embryos in the vial. Oxygen consumption is presented as $\mathrm{nmol} \mathrm{O}_{2} \mathrm{~h}^{-1}$ embryo- $^{-1}$.

\section{Statistical analysis}

Graphical and statistical analyses were performed using Prism 8.0 software (GraphPad, La Jolla, CA, USA) and SPSS software (IBM., v.26.0, Armonk, NY, USA). LT 50 of embryos exposed to dehydration was calculated for each developmental stage by probit regression analysis (Chapman et al., 1995) using SPSS software. Where appropriate, analysis of variance (ANOVA), unpaired t-test, or linear regression analyses were used. Tukey's honest significant different (HSD) test was used for post-hoc comparisons, where applicable. Statistical significance was set to $P<0.05$ for all comparisons. 


\section{Results}

\section{Tolerance of aerial exposure}

Embryos of $A$. limnaeus exhibited a profound tolerance of aerial conditions (85\% RH). This tolerance peaked in Dll embryos, with $10 \%$ surviving over $500 \mathrm{~d}$ and a single embryo living for $587 \mathrm{~d}$ (Fig. 3.1A). Remarkably, all developmental stages had embryos survive longer than $100 \mathrm{~d}$. When the survival data were used to calculate lethal time to $50 \%$ mortality $\left(\mathrm{LT}_{50}\right)$, Dll embryos exhibited an $\mathrm{LT}_{50}$ of $325 \pm 17 \mathrm{~d}$ (mean \pm SEM), which was significantly higher than the postDIl stages (Fig. 3.1B, one-way ANOVA with Tukey's HSD, $P<0.0001)$. Wourms' stage 36 embryos exhibited an $L_{50}$ of $84 \pm 5 d$, which was significantly higher than later stage embryos which had similar LT50 values of $28 \pm 6 d$ (WS 40) and $29 \pm 5$ d (WS 42) (Fig. 3.1B, one-way ANOVA with Tukey's HSD, $P<0.05)$. Embryos were not observed under a microscope, but macroscopic observation showed no eye development nor pigmentation in DII embryos, suggesting that embryos remained in diapause for the duration of the experiment.

\section{Metabolic rate}

Diapause II

Diapause II embryos consumed available oxygen in aqueous vials faster than aerial vials (Fig. 3.2A). However, because total oxygen available was 33 times lower in water compared to air, aerial exposed embryos consumed more available oxygen per unit time than aqueous exposed embryos (Fig 3.2C). Oxygen decreased in a linear fashion in trials using DII embryos, and thus a 
single linear regression was performed on each replicate to calculate the overall metabolic rate. To calculate rate of oxygen consumption, linear regressions were performed for each biological replicate $(N=4)$ for aerial and aqueous treatments (Fig 3.2E; Table 3.1). Rates of oxygen consumption for DIl embryos reported here (Fig 3.3A; $0.15 \pm 0.01 \mathrm{nmol} \mathrm{hr}^{-1}$ embryo- $^{-1}$, mean \pm SEM, $N=4$ ) are similar (unpaired t-test, $P=0.73)$ to those from previous experiments $(0.14 \pm 0.01 \mathrm{nmol}$ $\mathrm{hr}^{-1}$ embryo $^{-1}$, mean $\pm \mathrm{SEM}, N=4$ (Podrabsky and Hand, 1999)). Diapause II embryos exposed to aerial conditions had a rate of oxygen consumption that was over 4 times higher than those exposed to aqueous conditions (Fig 3.3A, Table 3.1; unpaired t-test, $P=0.0005)$. Diapause II embryos remained in diapause for the duration of the experiment in both aerial and aqueous treatments.

Post-diapause II (Wourms' stage 36)

All embryos began respirometry experiments at Wourms' stage 36. Initial rates of oxygen consumption in WS 36 embryos are similar in aerial versus aqueous conditions (Fig 3.3B; one-way ANOVA with Tukey's HSD test, $P=$ $0.9999)$. Mean rates of oxygen consumption for aqueous $\left(1.2 \pm 0.04 \mathrm{nmol} \mathrm{hr}^{-1}\right.$ embryo- $\left.^{-1}\right)$ and aerial $\left(2.3 \pm 0.41 \mathrm{nmol} \mathrm{hr}^{-1}\right.$ embryo- $\left.^{-1}\right)$ WS 36 embryos reported here are also similar (ANOVA, $P=0.07$ ) to those reported previously for aqueous embryos (1.6 $\pm 0.25 \mathrm{nmol} \mathrm{hr}^{-1}$ embryo-$^{-1}$ (Podrabsky and Hand, 1999)). The duration of the aqueous exposures in WS 36 embryos was short due to exhaustion of oxygen in the vials - nearly all oxygen was consumed after $72 \mathrm{~h}$ (Fig 3.2B). For aqueous exposures, a single linear regression was performed for 
the initial $72 \mathrm{~h}$ of each replicate (Fig 3.2F; Table 3.1). Post-diapause II embryos continued to develop at different rates during aerial exposures (Fig 3.3C) leading to a divergence in oxygen consumption rates between replicates over time (Fig 3.2F, 3.3C). For aerial incubations there was a clear initial rate of oxygen consumption early in the treatments that transitioned to much higher rates later in the trial, and thus two linear regressions were performed on each vial to maximize R squared values for initial (WS 36) and final (WS 38-42) rates of oxygen consumption (Fig 3.2F; Table 3.1). For aerial embryos, initial oxygen consumption includes the first $69 \mathrm{~h}$ and final rates were calculated using data between 165 and $452 \mathrm{~h}$. The timepoints between $69 \mathrm{~h}$ and $165 \mathrm{~h}$ were omitted from the regression analyses because oxygen content did change in a linear fashion. Time points after $452 \mathrm{~h}$ were omitted because oxygen content in the vials was below the measured critical oxygen level $\left(\mathrm{P}_{\text {crit }}\right)$ of $A$. limnaeus latestage embryos (Podrabsky and Wilson, 2016). Interestingly, there was a noticeable difference in the amount of oxygen remaining in each replicate vial in the aerial treatment, ranging from 4 to $13 \mu \mathrm{mol}$ per vial. However, long-term aerial incubated embryos had a significantly higher rate of oxygen consumption than initial rates observed in aerial and aqueous treatments, as would be expected for embryos that continue to develop (Fig 3.3B; one-way ANOVA with Tukey's HSD test, $P<0.01$ ). Mean rates of oxygen consumption in aerial WS 42 embryos that developed synchronously and continuously (the first 3 vials in Fig 3.3C; $6.14 \pm 0.12 \mathrm{nmol} \mathrm{hr}^{-1}$ embryo- $^{-1}$ ) were significantly lower (unpaired t-test, $P$ 
$=0.003)$ compared to those reported from previous studies $\left(16 \pm 1.5 \mathrm{nmol} \mathrm{hr}^{-1}\right.$ embryo $^{-1}$ (Podrabsky and Hand, 1999)).

\section{Discussion}

Despite knowledge of the unprecedented ability of annual killifish embryos to survive long periods of aerial exposure, this is the first time that $L T_{50}$ calculated values have been measured under controlled conditions across development. The ability of these embryos to survive in $85 \% \mathrm{RH}$ for hundreds of days is impressive for an aquatic embryo especially considering that $85 \% \mathrm{RH}$ is equivalent to being bathed in a solution of around 8000 mOsmol kg-1 salt (around $8 \mathrm{X}$ sea water). This ability is almost unparalleled in any other aquatic species and certainly suggests unique adaptations for preventing evaporative water loss. In addition, this is the first time that aerial oxygen consumption has been measured in A. limnaeus embryos. We hypothesized that aerial incubation would limit gas exchange with the environment in concert with the observed decrease in evaporative water loss (Podrabsky et al., 2001) and lead to self-imposed hypoxia or anoxia. While results for late-stage embryos are consistent with this hypothesis, DIl embryos respond in an opposite manner. This leads to an especially interesting question of how these embryos increase their rate of oxygen consumption while simultaneously reducing evaporative water loss to near zero and supporting survival for over a year under extremely dehydrating conditions. These unique responses to dehydration in dormant versus developing embryos may have important implications for the ecology of embryos in the wild. 
Rates of oxygen consumption reported here for aqueous conditions are similar to those reported for embryos of $A$. limnaeus from previous experiments (Podrabsky and Hand, 1999), despite the use of different respirometry techniques. Interestingly, when exposed to aerial conditions, DIl embryos have a higher rate of oxygen consumption compared to aqueous conditions. This is inconsistent with data from a species of non-annual killifish, Kryptolebias marmoratus, which exhibits a decrease in oxygen consumption under aerial incubation (Wells et al., 2015). These results were even more surprising given that even under aerobic aqueous conditions, DII embryos appear to support a large portion of their metabolism via anaerobic pathways (Podrabsky and Hand, 1999; Podrabsky et al., 2012a). Embryos incubated in aerial conditions did not break diapause and continue developing, which suggests either an energetic cost of survival of aerial conditions, or a diffusion-limited metabolism under aqueous conditions.

Annual killifish embryos, like all fish embryos prior to hatching, are limited to diffusive gas exchange with their environment. Under conditions of stagnant water flow, this can lead to impressively large boundary layers (up to $5 \mathrm{~mm}$ ) that may limit rates of diffusion (DiMichele and Powers, 1984). Further, annual killifishes possess an egg envelope that lacks pores and is thicker than in most species of fish (Schoots et al., 1982), which may also contribute to limited rates of diffusion. Thus, perhaps the greater oxygen consumption observed in aerial DIl embryos is due to improved rates of gaseous diffusion in an aerial environment as a consequence of a reduced effective boundary layer and the 
greater availability of oxygen in air compared to water. When exposed to aerial conditions, embryos of $A$. limnaeus lose most of the water in the perivitelline space within the first week (Podrabsky et al., 2001), which effectively brings the egg envelope closer to the fully hydrated embryonic compartment and may further reduce the diffusion distance for gas exchange. The diffusion coefficients through the egg envelope under hydrated and aerial conditions are unknown and would need to be determined to evaluate if diffusion may be limiting gas exchange in DIl embryos under aqueous conditions. However, this explanation is unlikely given data from other species (DiMichele and Powers, 1984) and the extremely low metabolic rates for DII embryos compared to post-diapause II embryos of $A$. limnaeus that do not exhibit an increase in oxygen consumption under aerial conditions. Thus, it is much more likely that the increased rate of oxygen consumption in DIl embryos represents the cost of a physiological response needed for survival of dehydrating conditions.

The long-term survival of DII embryos during aerial incubation (over a year) may require physiological responses to evaporative water loss such as membrane restructuring, accumulation of metabolites, and alteration of compounds in the perivitelline space. All of these responses would likely increase metabolic demand. The egg envelope, perivitelline space, and enveloping cell layer of annual killifish embryos likely work in concert to resist evaporative water loss and support survival of aerial conditions (Podrabsky et al., 2001) and molecular changes to these structures are of interest in explaining the extreme dehydration tolerance and lack of water loss over time. This could require the 
synthesis and accumulation of hydrophobic compounds in the membranes and perivitelline space, as suggested by Podrabsky et al. (2001). The soil dwelling collembolan Folsomia candida combats desiccation with a large increase in membrane mono-unsaturated fatty acids coupled with an increase of compatible osmolytes such as myo-inositol and glucose (Bayley and Holmstrup, 1999; Bayley et al., 2001). Similar observations were made in the Antarctic midge, Belgica antarctica, where dehydration led to increased fatty acid desaturase (FAD) gene expression, suggesting membrane restructuring via unsaturation (Lopez-Martinez et al., 2009). An increase in lipid content, especially neutral lipids, may be a mechanism to create lower resistance routes for oxygen diffusion into the embryo due to oxygen's higher solubility in lipids compared to water (Sidell, 1998). Unfortunately, little is known at the metabolic level in $A$. limnaeus embryos during aerial exposure, but results from this study suggest active mechanisms that support survival of long-term dehydration stress.

Initially, post-DII embryos (WS 36) of $A$. limnaeus maintained a similar rate of oxygen consumption in aerial and aqueous conditions. This result is consistent with previous reports for embryos of the non-annual killifish Fundulus heteroclitus (DiMichele and Powers, 1984; Tingaud-Sequeira et al., 2009). In contrast, WS 42 embryos of $A$. limnaeus have rates of oxygen consumption under aerial conditions that are over $60 \%$ lower than embryos under aqueous conditions. This result for late stage embryos is consistent with observations in K. marmoratus which experience a $44 \%$ reduction in oxygen consumption in response to aerial exposure (Wells et al., 2015). The differences in metabolic responses during 
initial and long-term dehydration for $A$. limnaeus is intriguing and may be due to developmental differences in response to dehydration stress, or to the experimental conditions used. For instance, in the present study, embryos were allowed to develop until DII under aqueous conditions and then transitioned into aerial incubation as diapause was broken, while for $K$. marmoratus the embryos experienced aerial conditions for the entirety of development. It is important to note that developmental rates of $A$. limnaeus begin to diverge at relatively high levels of oxygen that are above the measured $\mathrm{P}_{\text {crit }}$ of $A$. limnaeus embryos under aqueous incubation conditions (Podrabsky and Wilson, 2016). Thus, lower rates of oxygen consumption are not likely due to an acute response to hypoxia, but more likely represent a physiological response to a mixture of dehydration and hypoxia.

In contrast to other species of fish examined which tend to develop synchronously under aerial conditions (DiMichele and Powers, 1984; TingaudSequeira et al., 2009; Wells et al., 2015), development in A. limnaeus tends to be asynchronous after a week of aerial incubation. While the majority of the embryos continued developing similar to their aqueous counterparts, a subset of the embryos in four of the experimental vials remained at WS 38 from day 7 until the completion of the experiment $12 \mathrm{~d}$ later. Embryos of $A$. limnaeus enter quiescence in response to anoxia (Podrabsky et al., 2007), but this is the first report of quiescence-like behavior in this species in response to aerial conditions. It is likely that this stalling of development may be due to a combination of mild hypoxia and aerial exposure, because embryos exposed to $85 \% \mathrm{RH}$ in large 
containers where oxygen is not limited tend to develop synchronously (D.E.Z., personal observation). Of great interest is the apparent intrinsic variation observed in response to the aerial incubation conditions, which leads to developmental arrest in only a proportion of embryos.

The asynchronous development observed in post-DII embryos of $A$. limnaeus in response to aerial incubation could have profound influences on the developmental ecology of this species. Creating a pool of embryos in the soil at different developmental stages could be advantageous for persistence of the species by reducing the probability of total recruitment failure (Polačik et al., 2020). In contrast, synchronous development may lead to all embryos hastily responding to the same cue in a similar manner. Thus, this could represent a form of intrinsic bet-hedging and a source for added variation in the egg bank (Furness et al., 2015). Furness suggests that evolutionary bet-hedging guarantees that a portion of the embryos will develop successfully for a given set of actual conditions. The stress tolerance of $A$. limnaeus peaks at DII and decreases with post-DII development, as seen with anoxia, salinity, oxidative stress, and now dehydration stress (Machado and Podrabsky, 2007; Podrabsky et al., 2007; Riggs and Podrabsky, 2017; Wagner et al., 2019). By slowing postDII development during aerial exposure, embryos maintain greater stress tolerance, which may be crucial for survival until favorable conditions return. It is likely that $A$. limnaeus populations survive the dry seasons through entrance into DIl (Podrabsky et al., 2016; Podrabsky et al., 2001). A survey of African annual killifish embryos of the genus Nothobranchius in the field showed 
an egg bank primarily of DII embryos during the peak of dry season (Polačik et al., 2020). This is consistent with the remarkable tolerance of $A$. limnaeus embryos in DII to aerial conditions. However, despite post-DII embryos having less tolerance to dehydration stress than DII embryos, all stages measured survived over $100 \mathrm{~d}$ in aerial conditions, which may be long enough to survive a typical dry season if soil conditions remain at $\mathrm{RH}$ values of $85 \%$ or greater. That being said, much remains unknown about development under aerial conditions. Though not observed in these experiments, and not observed in the wild, DII embryos have been seen to break DII during aerial incubation (J.E.P., personal observations). The rate at which embryos develop after breaking DII under aerial incubation is unknown and is worth exploring. Additionally, exploration into the developmental rate of aerially incubated post-DII embryos when oxygen limitation is not a factor is of interest.

In summary, we show for the first time that embryos of $A$. limnaeus can survive aerial incubation for well over a year. Our findings are consistent with expected life history traits and known stress tolerance across development in $A$. limnaeus. Comparing aerial and aqueous oxygen consumption rates yielded unexpected outcomes and provide supporting evidence for the unique physiology of the annual killifish embryos in response to dehydration stress when compared to other fishes. Much remains unknown about survival of $A$. limnaeus embryos during aerial incubation. Further exploration at the metabolic level may provide insight into how this species survives in a dry substrate where they spend the majority of their lifecycle. 
Table 3.1. Oxygen consumption $\left(\mathrm{VO}_{2}\right)$ of Austrofundulus limnaeus embryos incubated in aerial or aqueous conditions

\begin{tabular}{|c|c|c|c|c|c|c|c|}
\hline \multirow{3}{*}{ Replicate } & \multirow{3}{*}{ Parameter } & \multicolumn{2}{|c|}{ Diapause II } & \multicolumn{4}{|c|}{ Post-diapause II } \\
\hline & & \multirow{2}{*}{$\begin{array}{l}\text { Aerial } \\
\text { Overall }\end{array}$} & \multirow{2}{*}{$\begin{array}{c}\text { Aqueous } \\
\text { Overall }\end{array}$} & \multicolumn{2}{|c|}{ Aerial } & \multicolumn{2}{|c|}{ Aqueous } \\
\hline & & & & WS 36 & WS $38-42$ & WS 36 & WS $38-42$ \\
\hline \multirow{3}{*}{1} & $\mathrm{VO}_{2}\left(\mathrm{nmol} \mathrm{O}_{2} \mathrm{~h}^{-1}\right.$ embryo- $\left.^{-1}\right)$ & 0.495 & 0.148 & 0.69 & 6.36 & 1.11 & \multirow{3}{*}{$\mathrm{n} / \mathrm{a}$} \\
\hline & $\mathrm{R}^{2}$ & 0.94 & 0.99 & 0.86 & 0.97 & 0.95 & \\
\hline & P-value & $<0.0001$ & $<0.0001$ & 0.0075 & 0.0017 & $<0.0001$ & \\
\hline \multirow{3}{*}{2} & $\mathrm{VO}_{2}\left(\mathrm{nmol} \mathrm{O} \mathrm{h}^{-1}\right.$ embryo $\left.^{-1}\right)$ & 0.582 & 0.170 & 1.82 & 6.11 & 1.13 & \multirow{3}{*}{$\mathrm{n} / \mathrm{a}$} \\
\hline & $\mathrm{R}^{2}$ & 0.92 & 0.97 & 0.98 & 1.00 & 0.96 & \\
\hline & P-value & $<0.0001$ & $<0.0001$ & 0.0001 & 0.0001 & $<0.0001$ & \\
\hline \multirow{3}{*}{3} & $\mathrm{VO}_{2}\left(\mathrm{nmol} \mathrm{O}_{2} \mathrm{~h}^{-1}\right.$ embryo $\left.^{-1}\right)$ & 0.610 & 0.164 & 0.34 & 5.58 & 1.20 & \multirow{3}{*}{$\mathrm{n} / \mathrm{a}$} \\
\hline & $\mathrm{R}^{2}$ & 0.99 & 0.96 & 0.99 & 0.99 & 0.98 & \\
\hline & P-value & $<0.0001$ & $<0.0001$ & $<0.0001$ & $<0.0001$ & $<0.0001$ & \\
\hline \multirow{3}{*}{4} & $\mathrm{VO}_{2}\left(\mathrm{nmol} \mathrm{O} \mathrm{h}^{-1}\right.$ embryo $\left.^{-1}\right)$ & 0.823 & 0.120 & 0.66 & 4.69 & 1.33 & \multirow{3}{*}{$\mathrm{n} / \mathrm{a}$} \\
\hline & $\mathrm{R}^{2}$ & 0.99 & 0.98 & 0.88 & 0.99 & 0.97 & \\
\hline & P-value & $<0.0001$ & $<0.0001$ & 0.0005 & 0.0005 & $<0.0001$ & \\
\hline \multirow{3}{*}{5} & $\mathrm{VO}_{2}\left(\mathrm{nmol} \mathrm{O}_{2} \mathrm{~h}^{-1}\right.$ embryo $\left.^{-1}\right)$ & & & 1.71 & 3.58 & 1.11 & \multirow{3}{*}{$\mathrm{n} / \mathrm{a}$} \\
\hline & $\mathrm{R}^{2}$ & & & 0.92 & 0.99 & 0.94 & \\
\hline & P-value & & & 0.0025 & 0.0004 & $<0.0001$ & \\
\hline \multirow{3}{*}{6} & $\mathrm{VO}_{2}\left(\mathrm{nmol} \mathrm{O} \mathrm{O}^{-1}\right.$ embryo $\left.^{-1}\right)$ & & & 1.046 & 1.12 & & \\
\hline & $\mathrm{R}^{2}$ & & & 0.94 & 0.95 & & \\
\hline & P-value & & & 0.0012 & 0.0048 & & \\
\hline \multirow{3}{*}{7} & $\mathrm{VO}_{2}\left(\mathrm{nmol} \mathrm{O}_{2} \mathrm{~h}^{-1}\right.$ embryo $\left.^{-1}\right)$ & & & 2.05 & 5.95 & & \\
\hline & $\mathrm{R}^{2}$ & & & 0.98 & 0.99 & & \\
\hline & P-value & & & 0.0002 & 0.0006 & & \\
\hline
\end{tabular}




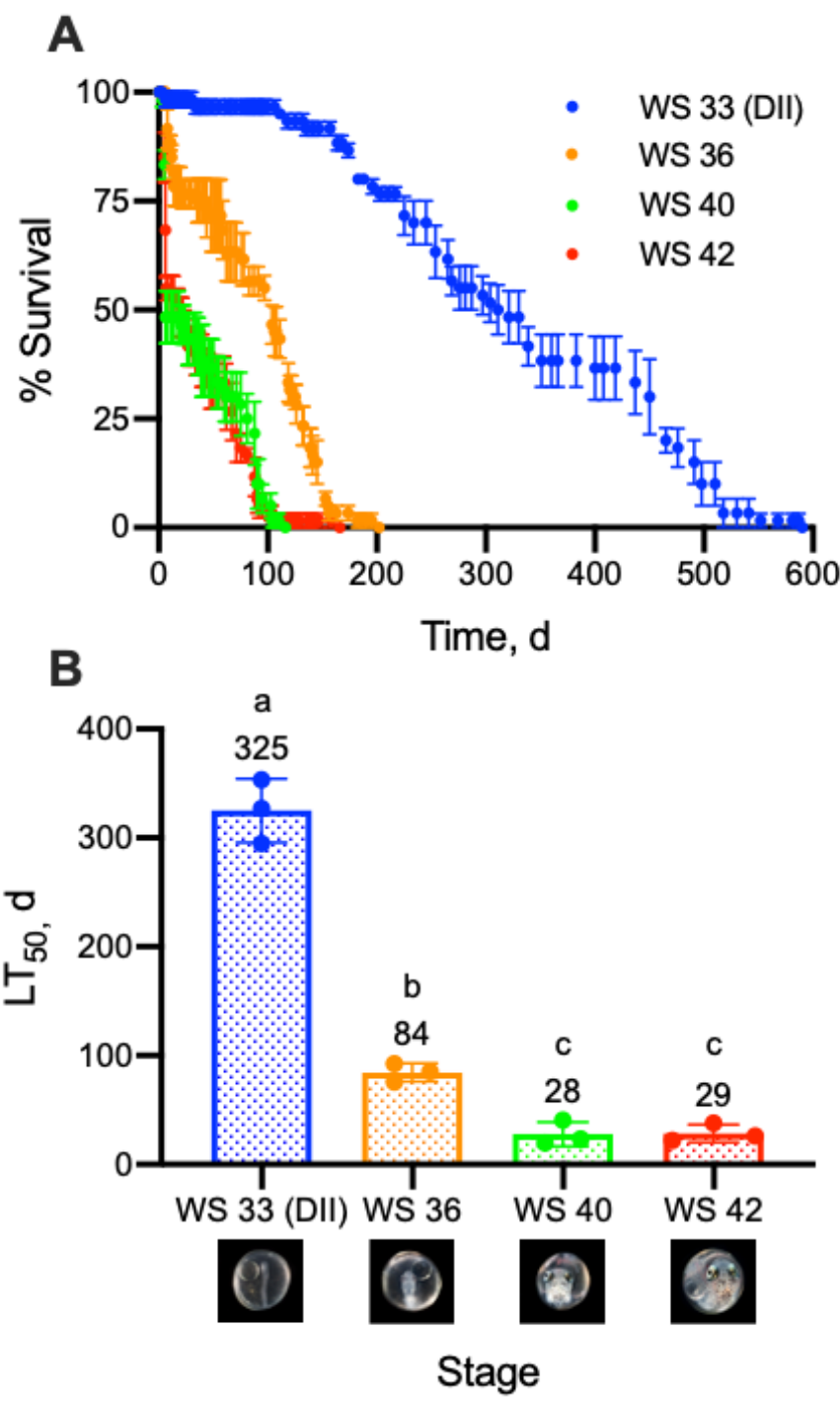

Figure 3.1. Survival of Austrofundulus limnaeus embryos incubated in aerial conditions. (A) Embryos were exposed to aerial conditions $(85 \% \mathrm{RH})$ at $25^{\circ} \mathrm{C}$ without light in groups of 20 starting at the developmental stage listed. Post-diapause II embryos continue to develop under aerial incubation conditions. WS, Wourms's stage; DII, diapause II. Symbols are means \pm SEM $(N=3)$. (B) Lethal time to $50 \%$ mortality $\left(\mathrm{LT}_{50}\right)$ for embryos exposed to aerial conditions. Different letters indicate significant differences in $\mathrm{LT}_{50}$ between developmental stages (one-way ANOVA with Tukey's HSD test, $P<0.05)$. Bars are means \pm SEM $(N=3)$ and symbols represent individual replicates. 

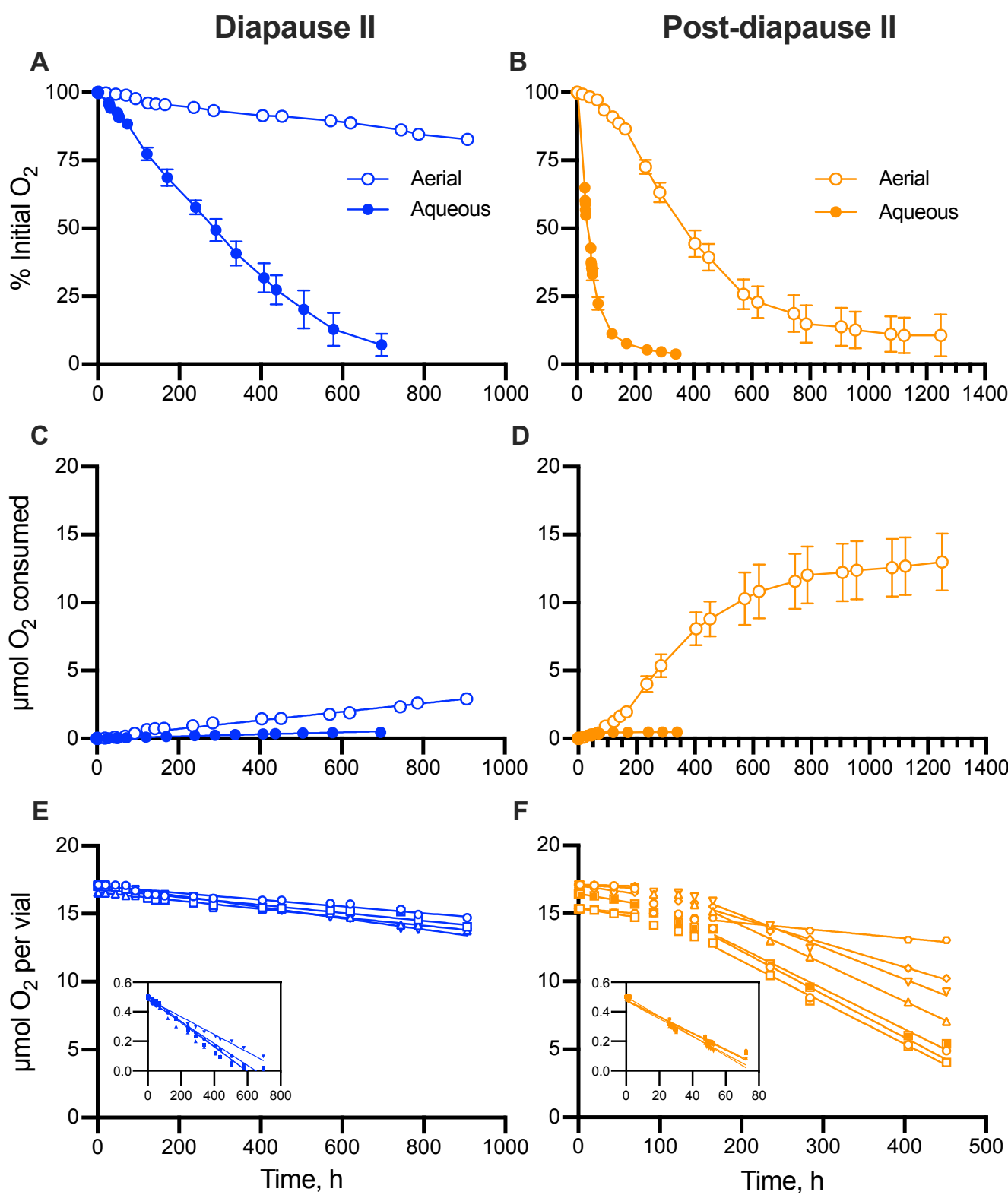

$\mathbf{F}$

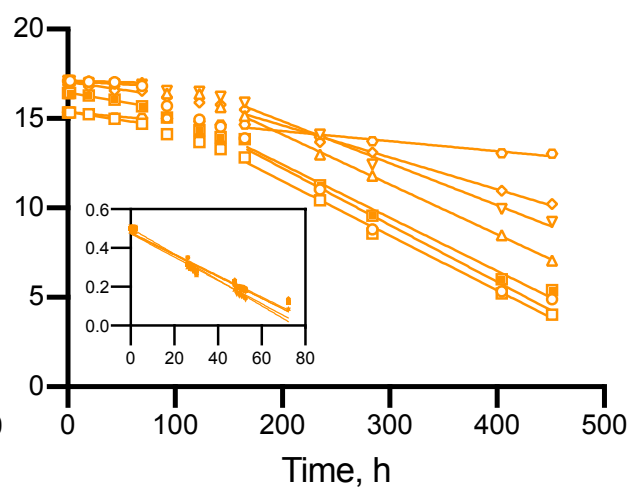

Figure 3.2. Oxygen consumption of Austrofundulus limnaeus embryos in aerial and aqueous conditions. (A,B) Percentage of initial oxygen in the closed system respirometry vials over time. Note: total initial oxygen content in aerial vials was 33 times more than that of aqueous vials. Symbols are means \pm SEM $(N=4-7)$ and each replicate contained 5 embryos per vial. $(C, D)$ The amount of oxygen $(\mu \mathrm{mol})$ consumed over time by embryos in aerial and aqueous treatments. (E,F) The amount of oxygen $(\mu \mathrm{mol})$ in each aerial exposure vial over time. Inset graphs are for aqueous conditions. Rates of oxygen consumption were based on the slope of oxygen consumed over time. For DII embryos, a single slope was used for the entire time-course. For post-diapause II embryos, an initial slope and a final slope were calculated. See Table 3.1 for results of regression analyses. 

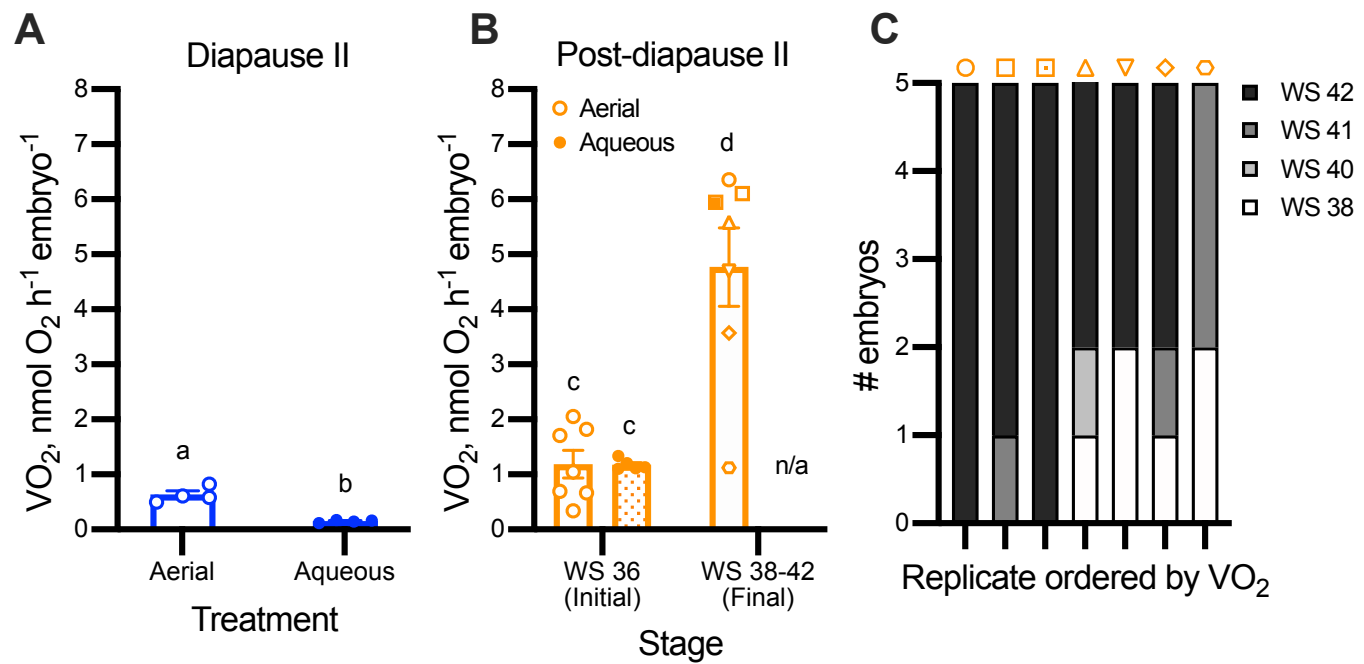

Figure 3.3. Rate of oxygen consumption in Austrofundulus limnaeus embryos exposed to aqueous and aerial conditions. (A) Diapause II embryos exposed to aerial conditions had a significantly higher rate of oxygen consumption than those exposed to aqueous conditions (unpaired t-test, $P=0.0005)$. Bars are means $\pm \operatorname{SEM}(N=4)$ and symbols represent individual replicates. (B) Wourms' stage 36 embryos (initial rate) exposed to aerial conditions had a mean rate of oxygen consumption similar to those incubated in aqueous conditions, although the variance is clearly higher in aerial conditions (one-way ANOVA with Tukey's HSD, $P=0.9999$ ). Final rates of oxygen consumption under aerial conditions in post-diapause II embryos varied considerably by replicate due to differences in developmental rates in embryos within each replicate as indicated by $(\mathbf{C})$ the developmental stage of embryos in each vial at the end of the experiment. Different letters indicate significant differences in rates of oxygen consumption (oneway ANOVA with Tukey's HSD test, $P<0.01$ ). For final measurements, symbols in $\mathbf{B}$ and $\mathbf{C}$ correspond to those used in Figure 3.2F. 


\section{Chapter 4}

\section{Metabolomics analysis provides insight on survival of annual killifish (Austrofundulus limnaeus) embryos during dehydration stress}

This chapter has previously been submitted for publication:

Zajic D, and Podrabsky J. Metabolomics analysis provides insight on survival of annual killifish (Austrofundulus limnaeus) embryos during dehydration stress. Physiol Genomics. Submitted: 2020.

\section{Introduction}

The annual killifish, Austrofundulus limnaeus, inhabits temporary mud puddles in the Maracaibo basin of Venezuela. Their habitat is defined by relatively predictable patterns of average seasonal precipitation, but highly unpredictable temporal and spatial patterns of episodic rain events. This pattern of water availability leads to a great deal of uncertainty in the length of time that a puddle will experience inundation. Thus, individual puddles may remain dry for months or perhaps years (Podrabsky et al., 2016; Podrabsky et al., 1998; Polačik and Podrabsky, 2015). In fact, individuals may spend the vast majority of life span as embryos encased in dry mud. During the rainy season, adult $A$. limnaeus spawn and deposit their embryos into the muddy and often oxygen-limited sediment. As the rainy season comes to an end, the adult fish die and embryos must survive severely dehydrating conditions, often faced with a variety of other stresses such as extremes in temperature and oxygen until the wet season 
returns and they can complete development (Podrabsky et al., 2016). As a result, A. limnaeus has evolved extremely stress-resistant embryos. Survival of these embryos is attributed, in part, to their ability to enter a profound state of metabolic depression termed diapause as part of their normal embryonic development (Podrabsky and Hand, 1999; Podrabsky and Hand, 2000). Entry into diapause allows for cessation of development, thus extending embryological survival during the dry season. There are three unique stages of diapause (I, II, and III) which an embryo can enter during development (Podrabsky et al., 2017; Wourms, 1972b; Wourms, 1972c). The most stress-tolerant stage, diapause II (DII), occurs midway through development and at this time the embryo mostly consists of cardiac and neural tissue (Podrabsky and Hand, 1999; Podrabsky et al., 2007). The developmental distribution of $A$. limnaeus embryos has not been characterized in the field. However, embryos of other species of annual killifish are primarily found in DII during the peak of the dry season, and in a variety of post-DII stages during the late dry season. Thus DII is likely primarily responsible for dry season survival in $A$. limnaeus, but dehydration tolerance is required during the entire duration of post-DII development. Even when embryos break DII and continue developing, they exhibit remarkable tolerance to stress that decreases as they progress towards hatching (Podrabsky et al., 2016; Podrabsky et al., 2001; Polačik et al., 2020).

Organismal survival in the absence of water is in itself a feat, but even more so for aquatic animals that rely on water as their main source of gas and nitrogenous waste exchange. Embryos of $A$. limnaeus experience unique 
resistance to desiccation that is not seen in other aquatic vertebrates (Podrabsky et al., 2001; Zajic et al., 2020). However, the desiccation tolerance of these embryos has received far less attention than other aspects of their biology. When embryos of $A$. limnaeus are exposed to dehydrating conditions, they initially respond by losing the water from their extraembryonic perivitelline space in the first few days before water loss is reduced and approaches near zero after a week (Podrabsky et al., 2001). Minimal water loss appears to occur within the embryonic compartment, and in fact the embryo appears to remain fully hydrated. Diapause II embryos of $A$. limnaeus can survive over 500 days of aerial exposure to $85 \%$ relative humidity $(\mathrm{RH})$ without access to liquid water, while post-DIl embryos can survive well over 100 days. Diapause II embryos respond to aerial incubation by increasing oxygen consumption. In contrast, postdiapause II (post-DII) embryos initially maintain similar levels of oxygen consumption and then experience a severe decrease in oxygen consumption later in development compared to their aqueous counterparts. The unique and unexpected responses to aerial incubation lead to questions about what is occurring at the molecular level to aid in survival. The remarkable survival of embryos during aerial incubation undoubtedly requires physiological responses to adapt to life without water that have long gone unexplored.

Although molecular oxygen is vital for organisms, it can have deleterious effects in excess (França et al., 2007). For aquatic organisms, aerial exposure represents a large increase in oxygen availability compared to most aquatic habitats. Thus, we may expect accumulation of metabolites during aerial 
exposure to assist in combating reactive oxygen species (ROS). A main reason for desiccation injury is attributed to the increased formation of ROS and subsequent oxidative damage due to water stress (França et al., 2007). Free radical formation can lead to lipid peroxidation, denaturation of proteins, and DNA damage which ultimately can affect overall metabolism (Hansen et al., 2006). However, protection against such damage can partially be mitigated by accumulation of antioxidant metabolites, such as glutathione (GSH) (Masella et al., 2005). Embryos of $A$. limnaeus have a notable tolerance of oxidative stress (Wagner et al., 2019), but the role of antioxidants has not been investigated through the lens of dehydration stress.

To survive without water, embryos must reduce evaporative water loss; accumulation of compatible osmolytes, restructuring of membranes, or alteration of waste compounds may therefore be critical for survival. A common mechanism for survival during water stress involves accumulation of water substitutes (e.g., trehalose) that can stabilize biological structures by "replacing" water and maintain hydrogen bonds that are critical for maintenance of structure (Erkut et al., 2011). This mechanism allows for the organism to enter a state of anhydrobiosis and is exhibited in a number of species, including tardigrades (Hypsibius dujardini), brine shrimp cysts (Artemia franciscana), and the dauer larva stage of Caenorhabditis elegans (Crowe et al., 1984; Erkut and Kurzchalia, 2015; Erkut et al., 2011). Although embryos of $A$. limnaeus cannot survive complete loss of cellular water, the accumulation of organic osmolytes is likely to be important in supporting survival of long-term dehydration stress. 
Austrofundulus limnaeus is a promising model organism for developmental physiology and ecology because of their tolerance of a plethora of stresses, annotated genome, and ability to alter developmental trajectories in the lab (Podrabsky et al., 2017; Podrabsky et al., 2010a; Romney et al., 2018; Wagner et al., 2018). The current study was performed to expand our knowledge of stress tolerance in A. limnaeus by elucidating the metabolic pathways that may be providing this species with its remarkable abilities to survive under extreme dehydration stress. We examine the metabolite profiles of dormant (DII) and actively developing (post-DII) embryos in response to short and long-term aerial incubation. Here we report abundant changes in the metabolite profiles of embryos exposed to dehydrating conditions. We show that actively developing and dormant embryos share metabolic pathways and accumulation of similar metabolites in response to dehydration. However, we also see developmentally distinct differences that suggest stage-specific responses to aerial incubation and dehydration stress. We show critical roles for amino acid and lipid metabolism that demonstrates embryos are not limited to carbohydrate reserves for survival. We identify known antioxidant and neuroprotective compounds, as well as unique metabolites that have not yet been seen in the dehydration literature. Our findings provide a novel view for understanding the long-term survival of aerially incubated embryos and provide a foundation for future functional studies. 


\section{Materials and Methods}

\section{Animal husbandry and embryo collection}

Adult annual killifish were housed and embryos collected as previously described (Podrabsky, 1999). All work was performed under established protocols that were reviewed and sanctioned by the Portland State University Institutional Animal Care and Use Committee (PSU IACUC protocols \#33 and 64). Briefly, adult fish were kept in male-female pairs and spawned semiweekly. Embryos were collected and stored at $25^{\circ} \mathrm{C}$ with no light in $15 \times 100 \mathrm{~mm}$ plastic Petri dishes in media that resembles the environmental conditions from which adults were collected in $1995\left(10 \mathrm{mmol} \mathrm{I}^{-1} \mathrm{NaCl}, 2.15 \mathrm{mmol} \mathrm{I}^{-1} \mathrm{MgCl}_{2}, 0.8 \mathrm{mmol} \mathrm{I}^{-1}\right.$ $\mathrm{CaCl}_{2}, 0.14 \mathrm{mmol} \mathrm{I}^{-1} \mathrm{KCl}, 1.3 \mathrm{mmol} \mathrm{I}^{-1} \mathrm{MgSO}_{4}$ ) (Podrabsky, 1999; Podrabsky et al., 1998). For the first 4 days post-fertilization (dpf), embryo medium contained methylene blue $(0.0001 \%)$ to prevent fungal infection. Embryos were then treated with two 5 min washes of a $0.01 \%$ sodium hypochlorite solution (separated by a 5 min rest in embryo medium) to prevent bacterial and fungal growth, as previously described (Podrabsky, 1999). Following sodium hypochlorite treatment, embryos were transferred to embryo medium containing $10 \mathrm{mg} \mathrm{l}^{-1}$ gentamicin sulfate and allowed to develop to DII (32-64 d). To break DII, embryos were subjected to a temperature of $30^{\circ} \mathrm{C}$ and full spectrum light for $48 \mathrm{~h}$ (Meller et al., 2012). Following this treatment, embryos were sorted into synchronized cohorts of embryos by developmental stage (Wourms' stage [WS]) as described in Podrabsky et al. (2017). 


\section{Embryonic stages investigated}

Experiments were performed on dormant (DII) and actively developing post-DII embryos (Podrabsky et al., 2017; Wourms, 1972b; Wourms, 1972c) to identify stage-specific responses to dehydration stress and to capture different levels of dehydration tolerance. DIl embryos are metabolically dormant, have halted development, and have a lethal time to $50 \%$ mortality $\left(\mathrm{LT}_{50}\right)$ in aerial incubation (85\% relative humidity $[\mathrm{RH}]$ ) of $325 \mathrm{~d}$ (Zajic et al., 2020). Dll embryos tend to stay in diapause during aerial exposure. Post-DIl embryos are metabolically active and continue developing during aerial exposure when oxygen is not limiting. Dehydration tolerance is reduced during post-DII development. When exposed to aerial conditions during early post-DII development (WS 36, 4 days post-diapause II), dehydration tolerance is reduced to an $L T_{50}$ of $84 \mathrm{~d}$. Late postDII embryos (WS 40-42) have a further reduced ability to survive aerial incubation and exhibit $L T_{50}$ values around 28-29 d (Zajic et al., 2020).

\section{Metabolomics analysis}

Aerial incubation

Embryos were exposed to $85 \%$ relative humidity $(\mathrm{RH})$ air at $25^{\circ} \mathrm{C}$ with no light in a sealed glass desiccator with a porcelain plate shelf $(250 \mathrm{~mm}$ diameter, $08615 \mathrm{~B}$, Fisher Scientific, Hampton, NH, USA). Relative humidity was controlled by using a saturated solution of potassium chloride $(750 \mathrm{ml})$ placed below the shelf and continually mixed with a stir bar to ensure uniform $\mathrm{RH}$ within the aerial portion of the chamber (Podrabsky et al., 2001; Winston and Bates, 1960). Prior to 
exposure, embryos were treated with sodium hypochlorite (see above) and incubated in embryo medium containing $10 \mathrm{mg} \mathrm{l}^{-1}$ gentamicin sulfate for $3 \mathrm{~h}$ prior to aerial exposure. Embryos were placed on filter pads containing $2.5 \mathrm{ml}$ embryo medium containing gentamicin. Care was taken to make certain that single embryos were isolated and not touching other embryos. DII embryos were then exposed to $85 \% \mathrm{RH}$ at $25^{\circ} \mathrm{C}$ without light and sampled at 0,7 , and $28 \mathrm{~d}$. Post-DII embryos were first exposed at WS 36 (4 days post-diapause II) and were sampled at 0,7 , and $18 \mathrm{~d}$. Post-DIl embryos continued developing during aerial incubation, which is consistent with previous data (Zajic et al., 2020). At 7 d, embryos sampled were at WS 40 and by $18 \mathrm{~d}$, embryos sampled were at WS 42/43. Six biological replicates $(N=6)$, comprised of 25 embryos each, were flash frozen with liquid $\mathrm{N}_{2}$ and stored at $-80^{\circ} \mathrm{C}$ until shipped to Metabolon for metabolite profiling. To better visualize the sampling regimen, see Figure 4.1.

\section{Metabolon metabolomics analysis}

Sample preparation and metabolomics analysis occurred at Metabolon, but is briefly detailed here. Samples were prepared using an automated MicroLab STAR system (Hamilton Company, Reno, NV, USA). Proteins were precipitated with methanol under vigorous shaking for 2 min (Glen Mills GenoGrinder 2000) followed by centrifugation. This method ensured dissociation of small molecules bound to protein, trapped in the precipitated protein matrix, and recovery of chemically diverse metabolites. The resulting extract was divided into four fractions: two for analysis by two separate reverse phase (RP)/UPLC-MS/MS 
methods with positive ion mode electrospray ionization (ESI), one for analysis by RP/UPLC-MS/MS with negative ion mode ESI, and one for analysis by HILIC/UPLC-MS/MS with negative ion mode ESI. To remove methanol, samples were placed briefly on a TurboVap (Zymark, Hopkinton, MA, USA). The sample extracts were stored overnight under nitrogen before preparation for analysis. Prior to analysis, sample extracts were reconstituted in solvents compatible to each of the four methods described above. Raw data was extracted, peakidentified, and quality control processed using Metabolon's proprietary hardware and software. At the time of analysis, identification of known biochemicals was based on comparison to metabolomic libraries of more than 3300 commercially available purified standard compounds. Several curation procedures were carried out to ensure high quality data and removal of those data representing system artifacts, mis-assignments, and background noise. The present dataset comprises a total of 673 compounds of known identity (metabolites). Data for each metabolite is presented relative to control samples $(t=0)$ and expressed as non-normalized, protein-normalized (Bradford assay), and DNA-normalized (Table 1). Due to the changing amount of water in the samples over the course of aerial incubation, and continued development in post-DII embryos, we have chosen to use the DNA-normalized data to most accurately reflect the relative amounts of metabolites per cell. Bradford protein concentration increases in a linear manner during dehydration while at the same time embryo mass decreases in a similar manner (Fig 4.2). We interpret this pattern as an artifact of 
water loss (especially in the DII embryos which are dormant) and so normalization per protein would exaggerate fold changes.

\section{Statistical analysis}

Graphical and statistical analyses were performed using Prism 8.0 software (GraphPad, La Jolla, CA, USA) or through Metabolon's user interface. Statistical significance was set to $P<0.05$ for all comparisons. For metabolomics data analysis, missing values due to being under the limit of detection of the instruments were imputed with the minimum value on a per metabolite basis. For each metabolite, raw counts were scaled to set the median across all samples for that metabolite to 1 and the data log transformed. Following normalization to Bradford protein or DNA concentration, Welch's two-sample $t$-test was used to identify metabolites that differed significantly between treatments, with a threshold for statistical significance set to $P<0.05$. Additionally, an estimate of the false discovery rate ( $q$-value) was calculated to take into account the multiple comparisons that normally occur in metabolomic-based studies. A low $q$-value ( $q$ $<0.10$ ) is an indication of high confidence in a result. Thus, only metabolites with $P<0.05$ and $q<0.10$ are considered significant in this study. Pathway enrichment analysis was performed using MetaboLync (Metabolon Inc.) with all metabolites and their pre-assigned pathways as background and reference pathways, respectively. Enriched pathways were calculated based on the following formula, where significance was defined as $P<0.05$ : 


$$
\text { Enrichment value }=(k / m) /((n-k) /(N-m))
$$

Where: $k$, total number of significant metabolites in pathway; $m$, total number of detected metabolites in pathway; $\mathrm{n}$, total number of significant metabolites; $\mathrm{N}$, total number of detected metabolites in the study. A pathway enrichment value greater than one indicates that the pathway contains more significantly changed compounds relative to the study overall. Fisher's exact test $(P<0.05)$ was used to determine if pathway enrichment was significant.

\section{Results and Discussion}

This is the first study to look at the metabolomic profile of embryos of $A$. limnaeus exposed to aerial incubation conditions. We previously measured the tolerance of DII and post-DII embryos to aerial conditions and showed the remarkable ability for DIl embryos to survive without access to liquid water for over $500 \mathrm{~d}$ (Zajic et al., 2020). The profound ability for embryos to survive over a year without water warranted an analysis of what is occurring at the metabolic level. It has been well-established that DII and post-DII embryos may respond to stress in a stage-specific manner (Podrabsky et al., 2012a; Riggs and Podrabsky, 2017). The metabolic profiles of DII and post-DII vary greatly, but due to continued development of post-DIl embryos during dehydration, we cannot confirm if the metabolites accumulated in post-DII embryos are dehydrationspecific or developmentally regulated. It is also important to note that the levels of metabolites reported in this paper are from whole embryos. The location of the metabolites, whether they be in the developing embryo, yolk, or perivitelline fluid, 
is not known. Recognizing the limitations of this study, we introduce new insights into survival of embryos of $A$. limnaeus to aerial incubation by focusing on pathways and metabolites that change in a similar manner in both dormant (DII) and actively developing (post-DII) embryos. Importantly, continuous exposure to dehydrating conditions during post-DII development is likely the most ecologically relevant condition under which to study this species. Below we outline metabolic pathways and metabolites of interest that may be supporting the extreme tolerance of embryos of $A$. limnaeus to dehydration stress induced by aerial exposure.

\section{Metabolomics profiles overview}

We measured a total of 673 metabolites of known identity (Supplemental Table S4.1). A summary of the numbers of metabolites that achieved statistical significance $(P<0.05)$ using various methods of normalization are presented in Table 4.1. Here we focus on data normalized to total DNA content to take into account the developmental changes that occur in post-DII embryos. We provide lists of the top significantly downregulated and upregulated metabolites in response to short-term or long-term aerial incubation in DII and post-DII embryos (Tables 4.2-4.9). Although the method of normalization is important, it does not affect the statistical significance of the vast majority of compounds that experience large fold-changes during aerial incubation (see boldface metabolites in Tables 4.2-4.9). Overall metabolite abundance was found to generally increase following aerial exposures, with the exception of short-term incubation 
of post-DIl embryos. DII embryos had 204 metabolites that were significantly altered during short-term exposures, while long-term exposures led to significant changes in 267 metabolites. Generally, metabolites (> 85\%) were upregulated under aerial conditions in DII embryos. Post-DII embryos had 553 metabolites that were altered during short-term exposures, of which 479 were downregulated. During long-term aerial incubation, 390 metabolites were altered, of which 212 were upregulated. These changes in metabolites, especially in dormant DII embryos, indicate a large-scale metabolic response to aerial conditions. The increase in the majority of measured metabolites in DII embryos suggests an active role in surviving dehydration stress. The increase in some metabolites is consistent with the observed rise in oxygen consumption seen in DIl embryos when exposed to aerial conditions (Zajic et al., 2020). Although dormant and not developing, DIl embryos appear to actively respond to dehydration stress induced by aerial incubation.

\section{Superpathways}

Metabolites were categorized as belonging to 1 of 8 superpathways (Fig 4.3). Of the metabolites measured, $70 \%$ belonged to lipid $(N=306)$ and amino acid $(N=164)$ metabolism. The remaining $30 \%$ belonged to nucleotide $(N=70)$, carbohydrate $(N=42)$, cofactors and vitamins $(N=30)$, xenobiotics $(N=30)$, peptide $(N=20)$, and energy metabolism $(N=11)$. Within each superpathway, metabolites were clustered based on 81 subpathways (Fig 4.3). 


\section{Enriched subpathways}

Diapause II.

There were 7 subpathways, primarily from lipid and amino acid metabolism, that were enriched in DII embryos following short-term aerial incubation (Table 4.9). The most enriched pathways were associated with fatty acid metabolism (dihydroxy, dicarboxylate, and monohydroxy). There was a significant contribution of amino acid metabolism, specifically, tyrosine metabolism, methionine, cysteine, SAM, and taurine metabolism, and leucine, isoleucine, and valine metabolism. The only pathway outside of amino acid and lipid metabolism that was enriched was nicotinate and nicotinamide metabolism. In response to long-term aerial incubation, there were 4 enriched subpathways ( 2 amino acid and 2 lipid) found in DIl embryos. The amino acid subpathways enriched are shared with those enriched during short-term exposure. Again, the most enriched pathway was fatty acid metabolism (acyl carnitine, long chain saturated). Another lipid metabolic pathway, monoacylglyercol metabolism, was also enriched.

\section{Post-DIl embryos.}

In general, there was more variation in pathway enrichment in post-DII embryos compared to DIl embryos (Table 4.9). There were 10 enriched subpathways in response to short-term $(N=5)$ and long-term $(N=5)$ aerial incubation. Most enriched pathways belonged to lipid and amino acid metabolism. There were three pathways that were consistently enriched during 
short and long-term exposures: phosphatidylcholine (PC), gamma-glutamyl amino acid, and monoacylglycerol metabolism. Unique to short-term incubation was enrichment in urea cycle (arginine and proline) metabolism and diacylglycerol metabolism. Unique to long-term exposure was enrichment in aminosugar metabolism and purine (adenine containing) metabolism.

\section{Shared metabolites (long-term dehydration)}

Due to continued development of post-DIl embryos during dehydration exposure, we chose to focus on metabolites that showed shared responses (upregulation or downregulation) in DII and post-DIl embryos in response to aerial exposure. Since DII embryos remained in DII during aerial incubation, we can more easily attribute metabolic change as primarily a direct response to dehydration stress and not an effect of active development. Thus, metabolites that change in both stages are likely due to a metabolic response to aerial incubation. DII embryos have a suppressed metabolism, thus changes in metabolic profiles may not be apparent after short-term incubation. We therefore chose to focus on metabolites shared following long-term aerial incubation to adequately capture a metabolic profile of embryos in response to dehydration stress.

When compared to their respective hydrated control, there were a total of 71 shared metabolites that significantly increased during long-term aerial incubation (Fig 4.4); whereas there were only 18 metabolites that significantly decreased during long-term exposures (Fig 4.4). Of the metabolites that 
increased, 48 belonged to lipid $(N=24)$ and amino acid $(N=24)$ metabolism.

The remainder belonged to carbohydrate $(N=8)$, nucleotide $(N=7)$, xenobiotics $(N=6)$, cofactors and vitamins $(N=1)$, and peptide metabolism $(N=1)$. Of the metabolites that decreased, a majority belonged to lipid metabolism $(N=11)$, and all lipid metabolites were from the monoacylglycerol subpathway. The remaining downregulated metabolites belonged to nucleotide $(N=2)$, cofactors and vitamins $(N=2)$, amino acid $(N=1)$, carbohydrate $(N=1)$, and xenobiotics $(N=$ 1) metabolism.

Many of these metabolites are in pathways found to be enriched in response to short or long-term aerial incubation (Table 4.9). In addition to those enriched pathways, there are several metabolites that belong to shared pathways that will be explored further. Below we introduce metabolites, sorted by superpathway and subpathway, that were significantly upregulated or downregulated in embryos in response to long-term aerial exposure that we will explore further.

\section{Pathways of interest}

Amino acids and dipeptides

Methionine, Cysteine, and Gamma-Glutamyl Amino Acids. There are indications of altered transsulfuration activity and antioxidant demand and utilization in DII and post-DII embryos exposed to aerial incubation (Fig 4.5). The transsulfuration pathway is a highly conserved pathway that is involved in metabolizing sulfur-containing amino acids such as methionine and cysteine. 
Methionine, via the transsulfuration pathway, can be converted to cysteine and several other compounds that play roles in redox balance and antioxidant defense (e.g., glutathione, taurine, etc.). Notably, an increase in the transsulfuration intermediate cystathionine is observed in both DII and post-DII embryos (Figs 4.4, 4.5).

The metabolite with the largest fold-change increase in response to aerial incubation is lanthionine (Fig 4.4; greater than 800 -fold increase in post-DII embryos). Lanthionine is formed when cysteine is metabolized in lieu of homocysteine or cystathionine by the transsulfuration pathway enzymes cystathionine $\beta$-synthase (CBS) and cystathionine $\gamma$-lyase (CSE) (Fig 4.5). A common product of the transsulfuration pathway, and specifically for lanthionine biosynthesis, is hydrogen sulfide $\left(\mathrm{H}_{2} \mathrm{~S}\right)$, which has been recognized as an important biological signaling molecule (Chiku et al., 2009; Elrod et al., 2007; Olson, 2018). $\mathrm{H}_{2} \mathrm{~S}$ has been shown to protect heart mitochondrial function during ischemic events and induce a state of suspended animation associated with a lower metabolism and body temperature in mice (Blackstone et al., 2005; Elrod et al., 2007). In addition, lanthionine can also be further metabolized in mammalian brain tissue to form the cyclic thioether, lanthionine ketimine, which is known to be associated with high affinity $(58 \mathrm{nmol})$ to neuronal membranes (Fontana et al., 1990; Ricci et al., 1989) and has been demonstrated to have neuroprotective, neurotrophic and anti-inflammatory activities in mice during cerebral ischemia (Hensley et al., 2010; Nada et al., 2012). Embryos of $A$. limnaeus may experience dehydrating conditions for months and perhaps years 
before water returns (Podrabsky et al., 2016; Polačik and Podrabsky, 2015).

Long-term survival of dehydrating conditions very likely depends on suppression of metabolism and induction of cellular protective mechanisms, either through entrance into diapause II, or through induction of a dehydration-induced quiescence (Zajic et al., 2020). Thus, it is possible that lanthionine accumulation may support long-term survival of dehydration stress through suppression of metabolism mediated by $\mathrm{H}_{2} \mathrm{~S}$ production, and through the cytoprotective actions of lanthionine ketimine in cardiac and neural tissues.

The large increase in the relative abundance of lanthionine suggests a potential role as a compatible osmolyte during conditions of water limitation in embryos of $A$. limnaeus. However, in the relatively few studies that exist, elevated lanthionine appears to be a toxin and to have generally negative effects on fish embryo physiology and development. When zebrafish (Danio rerio) embryos were treated with lanthionine $\left(0.3-2 \mu \mathrm{mol} \mathrm{I}^{-1}\right)$, abnormal development in heart chambers as well as increased heart rate and arrhythmia were observed, though some of these effects were counteracted with addition of glutathione (Perna et al., 2018). The potential toxic effects were further bolstered by evidence of lanthionine interfering with angiogenic signaling, increasing intracellular calcium, and impairing $\mathrm{H}_{2} \mathrm{~S}$ production (Perna et al., 2018; Vigorito et al., 2019). Thus, the ability of $A$. limnaeus embryos to tolerate such large increases in lanthionine without any apparent negative effects is an interesting result. Although we do not know the concentrations of lanthionine in embryos of A. limnaeus, an 800-fold change from even the picomolar level would reach 
biologically relevant concentrations as reported for $D$. rerio embryos. The biology of lanthionine, and its potential association with the production of $\mathrm{H}_{2} \mathrm{~S}$ warrants further investigation into its role during development and in response to dehydration stress in embryos of $A$. limnaeus.

Both the oxidized and reduced forms of glutathione (GSSG and GSH, respectively) were found to be accumulated in post-DII embryos, whereas only GSSG was increased in abundance in DII embryos. Cells use glutathione to scavenge reactive oxygen species (ROS) in order to reduce oxidative damage. A previous study showed a significant increase in total glutathione content in $A$. limnaeus embryos during post-DII development (Wagner et al., 2019). However, close to $100 \%$ of the glutathione measured was in its reduced form, thus the increases in GSSG observed in this study appear to be the result of aerial incubation. In their natural habitat, $A$. limnaeus embryos are exposed to a variety of conditions (e.g. hypoxia, high temperatures) as they endure the desiccating conditions of the dry season, which could lead to increases in ROS production. A rise in GSSG is usually indicative of some form of oxidative stress. One explanation for the observed rise in GSSG in DIl embryos may be due to increased oxidative stress as a consequence of increased oxygen availability and increased oxygen consumption observed in aerially incubated DII embryos (Zajic et al., 2020). Another possibility is that GSSG is assisting in reducing protein synthesis during dehydration, as GSSG is a known potent inhibitor of protein synthesis (Ernst et al., 1978). In response to dehydration, the star moss (Tortula ruralis) shows a similar rise in GSSG and subsequent decrease in protein 
synthesis. It is not until rehydration that GSSG is rapidly converted to GSH and protein synthesis resumes.

Post-DIl embryos also display significant decreases in 5-oxoproline, an intermediate of gamma-glutamyl amino acid metabolism, which could be reflective of changes in glutathione recycling. Changes in metabolites associated with the gamma-glutamyl amino acid subpathway were enriched during shortand long-term dehydration stress in post-DII embryos (Table 4.9). Fourteen gamma-glutamyl metabolites decreased in aerially incubated post-DII embryos (Fig 4.6). Only one metabolite, gamma-glutamyltryptophan, increased in both DII and post-DIl embryos following long-term dehydration, while 4 additional gammaglutamyl amino acids increased in DII embryos. There has been a lot of controversy in regards to the function of the gamma-glutamyl cycle in the literature. The original theory suggested the main function was transportation of amino acids into the cell via their conversion to gamma-glutamyl amino acids, a glutathione-dependent process (Orlowski and Meister, 1970). The release of amino acids from gamma-glutamyl amino acids by gamma-glutamyl cyclotransferase generates 5-oxoproline as an intermediate (Fig 4.5). However, recent findings suggest a role of the gamma-glutamyl cycle, not simply as an amino acid transporter, but as a regulator of redox metabolism of free radicals and xenobiotics (Inoue, 2016). A recent review by Bachhawat and Yadav proposes a "glutathione cycle" to replace the established gamma-glutamyl cycle (Bachhawat and Yadav, 2018). The discrepancies in these cycles is beyond the scope of this article, but there is a clear role for both glutathione and gamma- 
glutamyl metabolism in A. limnaeus embryos worth further exploration. The changes in these metabolites is consistent with potential alterations in transsulfuration activity and antioxidant mobilization in response to aerial incubation and dehydration stress.

Tryptophan Metabolism. There were 2 metabolites that increased in the pathway for metabolism of trytophan: C-glycosyltryptophan and picolinate. Of note is picolinate, which exhibited a profound fold-change (65-fold) in DII embryos and also a sequential increase in both stages during short- and longterm dehydration (Fig 4.4; Tables 4.2-4.5). The large fold-change increase observed in developmentally dormant embryos suggests an important role of picolinate. Picolinate has been found to have a wide range of neuroprotective, immunological, and anti-proliferative effects (Bosco et al., 2000; Fernandez-Pol et al., 1977; Grant et al., 2009; Ruffmann et al., 1987). Further investigations into a role for picolinate as a possible stress-induced cytoprotective molecule are a necessary next step in evaluating its potential importance.

Creatine Metabolism. DII and post-DII embryos exhibited different creatine metabolite profiles. Of note is the increase of creatine phosphate in DII embryos, and the sequential decrease of creatine phosphate in post-DII embryos in response to aerial exposure and dehydration stress (Fig 4.7). The reversible reaction of creatine to creatine-phosphate via creatine kinase generates diffusible high-energy phosphate reserves in the form of creatine phosphate. The 
accumulation of creatine phosphate in DII embryos indicates a positive energy balance and is consistent with previous reports of high ATP/ADP ratios and high adenylate energy charge values during diapause (Podrabsky and Hand, 1999). The decline in creatine phosphate and subsequent rise in creatine in post-DII embryos suggests that ATP demand is outpacing ATP production leading to the depletion of the creatine phosphate pool. This may suggest that aerial exposure is a metabolic stress for post-DII embryos and not for DII embryos. In addition to its role in buffering cellular ATP levels, creatine and creatine phosphate have been shown to exhibit a number of cytoprotective effects including membrane stabilization (Tokarska-Schlattner et al., 2012). Creatine phosphate can directly bind to and stabilize liposome membrane phospholipids by altering phase transition temperatures and reducing membrane leak (Tokarska-Schlattner et al., 2012). Additionally, it has been shown that phosphocreatine can have protective effects against ischemic injury (Landoni et al., 2016; Saks and Strumia, 1993). Thus, the accumulation of creatine phosphate in DIl embryos may offer protective effects that contribute to their much higher tolerance of aerial exposure and dehydration stress compared to post-DIl embryos.

Glutamate Metabolism. The only metabolite in the glutamate subpathway to be upregulated in both DII and post-DII embryos was gamma-aminobutyrate (GABA) (Fig 4.4; Table 4.5). GABA is known to accumulate during exposure to anoxia in embryos of $A$. limnaeus (Podrabsky et al., 2007; Zajic and Podrabsky, 2020). DII embryos exhibited a 1.6-fold increase in GABA while post-DII embryos 
showed a 20 -fold increase following long-term incubation. When GABA is measured spectrophotometrically, it is often undetectable during normal $A$. limnaeus development (Zajic and Podrabsky, 2020). Thus, the amount of GABA accumulating during dehydration stress probably represents micromolar quantities, and not millimolar levels as seen during anoxia (Zajic and Podrabsky, 2020). GABA, in the micromolar level, is known to have neuroprotective roles in organisms (Hylland and Nilsson, 1999; Nilsson et al., 1991). GABA has also been shown to be important in reducing ROS production and protecting against oxidative stress in yeast (Cao et al., 2013; Coleman et al., 2001). Although the levels are likely lower than during anoxia, GABA may still have an important role as a neuroprotective and antioxidant agent during exposures to dehydration.

\section{Carbohydrate}

Glycolysis, Gluconeogenesis, and Pyruvate Metabolism and the Pentose Phosphate Pathways. There were 4 metabolites from these pathways that increased in both DII and post-DII embryos following long-term dehydration: Glucose-6-phosphate, pyruvate, lactate, and sedoheptulose-7-phosphate (Figs 3,7). There were also two stage-specific metabolites that accumulated: DII embryos accumulated glycerate, whereas post-DII embryos accumulated phosphoenolpyruvate (PEP) (Fig 4.8; Tables 4.2-4.4). We previously hypothesized that aerial incubation would limit gas exchange with the environment in concert with the observed decrease in evaporative water loss (Podrabsky et al., 2001) and lead to self-imposed hypoxia or anoxia. Although we 
did observe results supporting this hypothesis in post-DIl embryos, DII embryos respond in an opposite manner by increasing oxygen consumption (Zajic et al., 2020). This previous result is supported by the relatively small accumulation of lactate observed in DIl embryos compared to post-DII embryos in response to dehydration stress. Thus, these data are consistent with hypoxic metabolism in post-DII but not DIl embryos during long-term dehydration stress. Interestingly, the increase in lactate resembles that of the Warburg effect, which is commonly observed in many cancer cells, where enhanced glycolysis and lactate fermentation occur even in the presence of sufficient oxygen. Relying partially on anaerobic mechanisms may offset the reliance on aerobic metabolism which can ultimately lead to increases in ROS. Additionally, lactate in appropriate amounts has been shown to help regulate gene expression through inhibition of histone deacetylase activity and post-translational modifications of histones, act in a neuroprotective role by preventing excitotoxic cell death, and promote oxidative stress resistance (Berthet et al., 2009; Jourdain et al., 2016; Latham et al., 2012; Tauffenberger et al., 2019; Zhang et al., 2019). Thus, aerobic accumulation of lactate may help fuel a response to dehydration stress through a variety of mechanisms outside of its role in the maintenance of cytoplasmic redox balance.

The accumulation of glucose-6-phosphate and sedoheptulose-7phosphate (S7P) is consistent with activation of the pentose phosphate pathway (PPP; Fig 4.8) (Patra and Hay, 2014). Shunting of carbon into the PPP results in the production of NADPH used to support anabolic processes, and also critical for the regeneration of reduced glutathione (Minard and McAlister-Henn, 2001; 
Patra and Hay, 2014). Accumulation of S7P, but not glyceraldehyde-3-phosphate (G3P) suggests that the oxidative portion of the PPP is used to produce NADPH, but not all of the carbon is shuttled back into glycolysis and instead is allowed to accumulate as S7P. In addition, the accumulation of several ribose metabolism intermediates (Fig 4.4) is also consistent with increased flux through the PPP. This hypothesis provides a mechanism to support antioxidant capacity (see above discussion of glutathione) while allowing limited immediate carbon flow through glycolysis in the form of G3P, and accumulating a source of carbon (S7P) that could be readily utilized by glycolysis at a later time. These data suggest a critical role for the PPP in supporting aerial incubation and dehydration stress in A. limnaeus embryos.

\section{Lipids}

Fatty Acid, Dicarboxylate. There were 7 metabolites that increased following long-term dehydration: Glutarate (C5-DC), 3-methylglutarate/2methylglutarate, 2-hydroxyglutarate, adipate (C6-DC), 3-hydroxyadipate, maleate, and sebacate (C10-DC) (Fig 4.4). Of interest is the accumulation of known oncometabolite 2-hydroxyglutarate (2-HG) (Sulkowski et al., 2020; Ward et al., 2010). There are two enantiomers of 2-HG, (S)2-HG and (R)2-HG, each of which can inhibit alpha-ketoglutarate-dependent enzymes involved in many biological processes (Xu et al., 2011). The methodology used to measure 2-HG in this study cannot discern enantiomers, thus further exploration needs to be done to see which form of the metabolite is found in embryos. Mutations in 
isocitrate dehydrogenase associated with cancers produce $(\mathrm{R}) 2-\mathrm{HG}$, which can stimulate cancer cell proliferation and inhibit TET enzymes and histone demethylases (Charitou et al., 2015; Intlekofer et al., 2017). In contrast, (S)2-HG is produced during oxygen limitation to regulate histone methylation levels and to help mitigate cellular reductive stress through inhibition of glycolysis and electron transport in mammalian cells (Intlekofer et al., 2015; Oldham et al., 2015). Formation of (S)2-HG can be formed through lactate dehydrogenase (LDH) and malate dehydrogenase $(\mathrm{MDH})$ via 'promiscuous' reduction of the alternative substrate alpha-ketoglutarate (Intlekofer et al., 2015; Intlekofer et al., 2017). Independent of enantiomer-specificity, 2-HG has been shown to inhibit ATP synthase and extend lifespan of Caenorhabditis elegans (Fu et al., 2015). 2-HG can have a number of effects on $A$. limnaeus embryos that could be beneficial in supporting survival during aerial incubation and dehydration stress.

Fatty Acid, Acyl carnitine. Several acyl carnitine metabolites $(N=5)$ were upregulated: arachidoylcarnitine (C20), arachidonoylcarnitine (C20:4), docosapentaenoylcarnitine (C22:5n6), stearoylcarnitine (C18), palmitoleoylcarnitine (C16:1) (Fig 4.4). Acyl carnitines play an essential role in regulating the balance of intracellular sugar and lipid metabolism (Li et al., 2019). Acyl carnitines primarily serve a functional role as carriers to transport long-chain fatty acids into mitochondria for $\beta$-oxidation, which is a major source of energy for cellular activities (Li et al., 2019; Tarasenko et al., 2018). Accumulation of these 
metabolites may suggest reduced mitochondrial fatty acid metabolism during aerial incubation.

Phospholipids. The only metabolite in the phospholipid subpathway to be upregulated in both DII and post-DII embryos was cytidine 5'-diphosphocholine (Fig 4.4). Cytidine 5'-diphosphocholine is an intermediate product of phosphatidylcholine synthesis. The compound has been used extensively in clinical settings to treat patients with neurological disorders, including cerebral ischemia. The main protective role of cytidine $5^{\prime}$-diphosphocholine is through membrane stabilization (Zweifler, 2002). Further research has shown cytidine 5'diphosphocholine protects rat livers from ischemia and reperfusion injury, thus preserving mitochondrial function and reducing oxidative stress (Zazueta et al., 2018). Reducing damage to oxygen-sensitive tissue, such as neural tissue, by stabilizing cell membranes and reducing free radical generation may be beneficial to survival of $A$. limnaeus embryos during aerial exposures and in response to dehydration stress.

Bile Acid Metabolism. Both taurolithocholate and taurochenodeoxycholate accumulate during long-term aerial exposure. Taurolithocholate accumulates to higher levels in DIl embryos (almost 10-fold), while taurochenodeoxycholate exhibits an over 100-fold increase in post-DIl embryos (Fig 4.4). In general, bile acids are toxic due to their ability to act as detergents, but the tauro-derivatives of bile acids tend to be less toxic (Rust et al., 2000). It is interesting that DII 
embryos accumulate bile acids despite the absence of liver tissue. In addition to their role in digestion, bile acids can act as signaling molecules via a variety of pathways, including the regulation of a number of nuclear receptors (Hylemon et al., 2009). It is possible that these potentially toxic compounds are produced to activate signaling pathways that help support survival of aerial exposure. This possibility is intriguing and warrants further investigation.

\section{Cofactors and Vitamins}

Nicotinate and Nicotinamide metabolism. Metabolites linked to de novo and salvage (nicotinamide, nicotinamide riboside, nicotinamide ribonucleotide, and nicotinate) pathways of NAD+ synthesis were found to be significantly altered in response to aerial exposure in both DII and post-DII embryos. Of these, one metabolite was upregulated in both stages following long-term dehydration stress: nicotinamide (Fig 4.4). There were, however, 2 metabolites that were downregulated: nicotinate and nicotinamide ribonucleotide (NMN) (Fig 4.4). One difference between DII and post-DII embryos was the significant accumulation of nicotinamide adenine dinucleotide (NAD+) in Dll embryos (Fig 4.9; 17 -fold increase). NAD+ is a coenzyme that plays a vital role in redox reactions associated with glycolysis, the TCA cycle, and oxidative phosphorylation. Increasing NAD+ levels in mammals has been linked to improved mitochondrial function under stress (Canto et al., 2015; Cerutti et al., 2014; Khan et al., 2014). Enhancing NAD+ availability in C. elegans has been shown to increase lifespan and protect against ROS (Mouchiroud et al., 2013). 
Conversely, reduction in NAD+ levels is an indication of senescence, as seen in various animal cell lines (Braidy et al., 2011; Canto et al., 2015; Gomes et al., 2013; Mouchiroud et al., 2013). Synthesis of NAD+ can occur via salvage and de novo pathways (Fig 4.9). De novo synthesis of NAD+ can occur via tryptophan metabolism by converting quinolinate to nicotinate ribonucleotide (NAMN). There is an initial rise in quinolinate in DIl embryos followed by a decrease after longterm exposure. It is likely that the decrease in quinolinate contributed to the increase in NAD+ (Fig 4.9). The overall rise in NAD+ in Dll embryos and maintained levels in post-DII embryos following long-term dehydration stress suggest changes in both demand and synthesis. The accumulation of NAD+ in DII suggests an available pool of NAD+ to contribute to metabolism, while the static levels of NAD+ in post-DII embryos suggests supply and demand are in synergy. The rise in the associated metabolites in the pathway provide a molecular supply for further synthesis of NAD+ via the salvage pathway.

\section{Xenobiotics}

Xenobiotics refer to compounds not expected to be present within an organism. There were several metabolites that were upregulated which are traditionally seen interacting with the microbiome. There were 3 metabolites upregulated in the tyrosine subpathway: phenol sulfate, 4-

hydroxyphenylpyruvate, and 3-methoxytyrosine. Both DII and post-DII embryos exhibited an over 50-fold increase in phenol-sulfate following long-term dehydration (Fig 4.4). There were 3 metabolites in the benzoate pathway that 
were upregulated following long-term dehydration: hippurate, guaiacol sulfate, $p$ cresol sulfate. Hippurate exhibited the largest fold change (over 100-fold) in DII embryos (Fig 4.4). Of these metabolites, hippurate and p-cresol sulfate are known markers for microbiome diversity (Lees et al., 2013; Pallister et al., 2017). Hippurate, along with other benzoates, has been shown to inhibit the growth of tumors in mouse cell lines (Spustova and Oravec, 1989). Much remains unknown about the role of benzoate metabolism outside of the microbiome and as a urinary waste product. The accumulation of these metabolites in $A$. limnaeus is interesting, as it is currently unknown whether fish embryos contain a maternally packaged microbiome that would explain these metabolites; this possibility is intriguing. While it is possible that these compounds accumulate due to microbial growth on the surface of the embryos, this is unlikely given the use of antibiotics in the medium, the detection of these metabolites at time zero, and the lack of other more general microbially-derived compounds that would be expected to accumulate. In addition, we observe the accumulation of bile acid metabolites in embryos that have not yet developed functioning liver tissue. Thus, there are many compounds that accumulate during dehydration stress that are inconsistent with our current knowledge of the metabolic capabilities of annual killifish embryos. There is evidence of specific enzymes in the yolk sacs of chicken and tortoise embryos that are capable of producing metabolites that otherwise only exist in microorganisms and plants (Chapeville and Fromageot, 1967). For example, chicken embryos are able to synthesize lanthionine from sulfate with the help of enzymes exclusively found in yolk sac endodermal cells. These 
enzymes disappear upon hatching, which may help explain the lack of lanthionine in the literature. The possibility of these enzymes and metabolic capabilities in A. limnaeus is an exciting avenue worth further exploration. Alternatively, it is possible that these metabolites are produced by a yolk sac specific microbiome that aids in survival of dehydration stress. Further research needs to be done to elucidate the location, origin, and role of these metabolites in A. limnaeus embryos.

\section{Conclusions and Future Directions}

The goal of this metabolic profiling study was to gain biochemical insight into the mechanisms facilitating survival of aerial exposure and dehydration stress in A. limnaeus embryos. There is robust evidence of metabolic segregation in the study based on both embryonic stage and dehydration exposure, with changes following aerial exposure being significantly more pronounced in post-DIl embryos, as expected. It also appears as though diapause II embryos, despite being metabolically dormant, are actively responding to dehydration tolerance by altering their metabolic profiles. There was an overwhelming downregulation trend seen in metabolites during shortterm aerial exposure of post-DIl embryos, which may indicate an initial response to elevated oxygen availability in the short-term and progressive imposition of hypoxia in long-term responses to aerial exposure and dehydration stress. However, further studies need to be done to delineate whether the responses seen in the post-DIl embryos are developmental differences or dehydration- 
specific responses. Despite this, there are an abundance of metabolites that change together in DII and post-DII embryos that may serve promising functions for supporting survival during aerial incubation and dehydration. In general, amino acid and lipid metabolism appear to play central roles in metabolic adjustments during aerial exposure, and it is clear that embryos are not solely relying on carbohydrate reserves to fuel metabolism. Increased capacity for detoxification of ROS and maintenance of redox balance appear to both be of major importance to supporting development under aerial conditions, and surviving dehydration stress. It is also interesting that several of the metabolites that experience large fold-change increases have previously been identified as neuroprotectants. This work offers a first glimpse into the metabolic programs that may support survival of aerial incubation and long-term survival of dehydration stress in embryos of $A$. limnaeus that can be functionally tested using genetic and pharmacological approaches.

\section{Perspectives}

Embryos of $A$. limnaeus likely spend many months, the preponderance of their life span, aerially incubated in the wild. During aerial exposure, there are a number of metabolites accumulated that are usually associated with waste or have the potential to be toxic (e.g., bile acids, hippurate, lanthionine). Many of these compounds contain nitrogen or other typical waste products of metabolism that are excreted in the urine and feces. When exposed to aerial conditions, embryos of annual killifish must presumably rely on detoxification or 
compartmentalization of waste product metabolites to ensure the developing embryo remains unharmed. One possible compartment for storage of waste products is the yolk. We previously suggested that lactate accumulated during anoxia may be buffered by transportation to the yolk, which is supported by the expression of several lactate transporter transcripts (Zajic and Podrabsky, 2020). It is possible that metabolites accumulated during aerial exposure are being shuttled into the yolk to protect the developing tissues, as seen with sequestration of ammonia in the yolk sac of rainbow trout (Steele et al., 2001). In amniotic eggs, the eggs of terrestrial vertebrates, waste is transported from the developing embryo into extraembryonic compartments, notably the amnionic, and the allantoic fluid (Aktuğ et al., 1998; Leeson and Leeson, 1963; Mellor and Slater, 1971; Ten Busch et al., 1997; Williams et al., 1993). In the anamniotic eggs of fish, waste products such as ammonia or bile acids can be relatively easily lost due to diffusion into the perivitelline fluid during early development, and excretion during late development as the chorion is not a substantial barrier for diffusion of relatively small metabolites (Peterson and Martin-Robichaud, 1987; Wright et al., 1995). However, in A. limnaeus the perivitelline fluid is quickly lost during aerial exposures and can no longer function as a mechanism for diffusive loss of metabolic waste products. Indeed, the embryos of annual killifish embryos exposed to aerial incubation have several unique features that approximate the functions of the shell and extraembryonic components of the amniotic eggs of terrestrial species. First, the chorion of annual killifish lacks pores and is substantially thicker than in most species of fish (Schoots et al., 
1982), and thus serves a protective function and as a partial barrier for larger molecules even under aquatic conditions (Schoots et al., 1982). Second, the enveloping cell layer of annual killifish embryos does not contribute to the skin of the developing embryos as it does in most fishes, instead it forms a syncytial membrane that surrounds the embryo and is shed upon hatching (Wourms, 1972a). This membrane constitutes the major permeability barrier between the embryo and its environment and has a unique structure with few embedded proteins (Jorgenson and Schmalbruch, 1984) and an extremely low permeability to water and salts (Machado and Podrabsky, 2007). The enveloping layer in annual killifishes effectively creates an extra-embryonic membrane-bound compartment that surrounds and protects the embryo in a manner similar to an amniotic chamber in terrestrial eggs. Thus, it is possible that a number of the compounds that are typically excreted as waste may be stored in either the yolk sac, or in extraembryonic fluid that is bound by the enveloping cell layer. In fact, many of the accumulated compounds identified in this study are known components of amniotic and allantoic fluid in other species (Aktuğ et al., 1998; Mellor and Slater, 1971; Ten Busch et al., 1997; Williams et al., 1993). Further exploration into the localization of these metabolites will help elucidate their possible function during aerial incubation and survival of dehydration stress. 
Table 4.1. Total number of significant metabolites (among 673 total metabolites identified) that were significantly affected by short and long-term aerial exposure in DII and post-DII embryos initially exposed at WS 36 .

\begin{tabular}{|c|c|c|c|c|c|c|c|}
\hline \multirow[b]{2}{*}{ Stage } & \multirow[b]{2}{*}{ Comparison } & \multicolumn{2}{|c|}{$\underline{\text { Non-normalized }}$} & \multicolumn{2}{|c|}{ Protein-normalized } & \multicolumn{2}{|c|}{ DNA-normalized } \\
\hline & & $\begin{array}{c}\text { Total } \\
\text { Number } \\
P<0.05\end{array}$ & $\begin{array}{c}\text { Number } \\
\text { (up I } \\
\text { down) }\end{array}$ & $\begin{array}{c}\text { Total } \\
\text { Number } \\
P<0.05\end{array}$ & $\begin{array}{c}\text { Number } \\
\text { (up I } \\
\text { down) }\end{array}$ & $\begin{array}{c}\text { Total } \\
\text { Number } \\
P<0.05\end{array}$ & $\begin{array}{c}\text { Number } \\
\text { (up I } \\
\text { down) }\end{array}$ \\
\hline \multirow{2}{*}{ DII } & $\begin{array}{c}7 \mathrm{~d} / 0 \mathrm{~d} \\
\text { (short-term) }\end{array}$ & 243 & $156 \mid 87$ & 238 & $85 \mid 153$ & 204 & 174 | 30 \\
\hline & $\begin{array}{c}28 \mathrm{~d} / 0 \mathrm{~d} \\
\text { (long-term) }\end{array}$ & 346 & $298 \mid 48$ & 200 & $77 \mid 123$ & 267 & $233 \mid 34$ \\
\hline \multirow{2}{*}{$\begin{array}{l}\text { Post- } \\
\text { DII }\end{array}$} & $\begin{array}{c}7 \mathrm{~d} / 0 \mathrm{~d} \\
\text { (short-term) }\end{array}$ & 466 & $383 \mid 83$ & 438 & $269 \mid 169$ & 553 & $74 \mid 479$ \\
\hline & $\begin{array}{c}18 \mathrm{~d} / 0 \mathrm{~d} \\
\text { (long-term) }\end{array}$ & 558 & $430 \mid 128$ & 544 & $354 \mid 190$ & 390 & $212 \mid 178$ \\
\hline
\end{tabular}




\section{Table 4.2. Top 40 significantly upregulated metabolites during short-term aerial exposure}

in DIl embryos normalized to DNA concentration.

\begin{tabular}{|c|c|c|c|}
\hline Rank & Significant metabolite & $\begin{array}{l}\text { Metabolite } \\
\text { Fold } \\
\text { change }\end{array}$ & Associated sub pathway \\
\hline 1 & phenol sulfate & 39.52 & Tyrosine Metabolism \\
\hline 2 & tartarate & 24.84 & Food Component/Plant \\
\hline 3 & alpha-hydroxycaproate & 22.29 & Fatty Acid, Monohydroxy \\
\hline 4 & hippurate & 20.11 & Benzoate Metabolism \\
\hline 5 & picolinate & 16.79 & Tryptophan Metabolism \\
\hline 6 & $\begin{array}{r}\text { nicotinamide adenine dinucleotide } \\
\text { (NAD+) }\end{array}$ & 16.34 & Nicotinate and Nicotinamide Metabolism \\
\hline 7 & p-cresol sulfate & 14.51 & Benzoate Metabolism \\
\hline 8 & maleate & 9.91 & Fatty Acid, Dicarboxylate \\
\hline 9 & 3-indoxyl sulfate & 9.83 & Tryptophan Metabolism \\
\hline 10 & 1-carboxyethylphenylalanine & 9.75 & Phenylalanine Metabolism \\
\hline 11 & hydroquinone sulfate & 8.55 & Drug - Topical Agents \\
\hline 12 & guaiacol sulfate & 8.36 & Benzoate Metabolism \\
\hline 13 & lanthionine & 7.15 & $\begin{array}{l}\text { Methionine, Cysteine, SAM and Taurine } \\
\text { Metabolism }\end{array}$ \\
\hline 14 & salicylate & 7.05 & Drug - Topical Agents \\
\hline 15 & sebacate (C10-DC) & 6.97 & Fatty Acid, Dicarboxylate \\
\hline 16 & cysteine sulfinic acid & 6.9 & $\begin{array}{l}\text { Methionine, Cysteine, SAM and Taurine } \\
\text { Metabolism }\end{array}$ \\
\hline 17 & quinolinate & 6.74 & Nicotinate and Nicotinamide Metabolism \\
\hline 18 & taurolithocholate & 6.24 & Secondary Bile Acid Metabolism \\
\hline 19 & 2-aminoheptanoate & 5.89 & Fatty Acid, Amino \\
\hline 20 & arabinose & 5.66 & Pentose Metabolism \\
\hline 21 & 1-methyl-4-imidazoleacetate & 4.81 & Histidine Metabolism \\
\hline 22 & benzoate & 4.5 & Benzoate Metabolism \\
\hline 23 & glutarate (C5-DC) & 4.37 & Fatty Acid, Dicarboxylate \\
\hline 24 & N-palmitoyltaurine & 3.95 & Endocannabinoid \\
\hline 25 & undecanedioate (C11-DC) & 3.63 & Fatty Acid, Dicarboxylate \\
\hline 26 & benzoylcarnitine* & 3.52 & Chemical \\
\hline 27 & glycerate & 3.36 & $\begin{array}{l}\text { Glycolysis, Gluconeogenesis, and Pyruvate } \\
\text { Metabolism }\end{array}$ \\
\hline 28 & glucuronate & 3.34 & Aminosugar Metabolism \\
\hline 29 & ethylmalonate & 3.33 & Leucine, Isoleucine and Valine Metabolism \\
\hline 30 & 2-hydroxyheptanoate* & 3.26 & Fatty Acid, Monohydroxy \\
\hline 31 & phytosphingosine & 3.21 & Sphingolipid Synthesis \\
\hline 32 & 1-methyl-5-imidazoleacetate & 3.16 & Histidine Metabolism \\
\hline 33 & isovalerylcarnitine (C5) & 3.07 & Leucine, Isoleucine and Valine Metabolism \\
\hline 34 & adipate (C6-DC) & 3.02 & Fatty Acid, Dicarboxylate \\
\hline 35 & 2-hydroxypalmitate & 3 & Fatty Acid, Monohydroxy \\
\hline 36 & 4-hydroxyphenylpyruvate & 2.93 & Tyrosine Metabolism \\
\hline 37 & pyruvate & 2.93 & $\begin{array}{l}\text { Glycolysis, Gluconeogenesis, and Pyruvate } \\
\text { Metabolism }\end{array}$ \\
\hline 38 & cysteine & 2.92 & $\begin{array}{l}\text { Methionine, Cysteine, SAM and Taurine } \\
\text { Metabolism }\end{array}$ \\
\hline 39 & tartronate (hydroxymalonate) & 2.92 & Food Component/Plant \\
\hline 40 & N-oleoyltaurine & 2.88 & Endocannabinoid \\
\hline
\end{tabular}

Metabolites in bold are also found in the top 40 when normalized to protein content. Metabolites with asterisks indicate compounds that have not been confirmed based on standards, but Metabolon is confident in their identity. 
Table 4.3. Top 40 significantly upregulated metabolites during long-term aerial exposure in DIl embryos normalized to DNA concentration.

\begin{tabular}{|c|c|c|c|}
\hline Rank & Significant metabolite & $\begin{array}{c}\text { Metabolite } \\
\text { Fold } \\
\text { change }\end{array}$ & Associated sub pathway \\
\hline 1 & hippurate & 101.49 & Benzoate Metabolism \\
\hline 2 & picolinate & 64.9 & Tryptophan Metabolism \\
\hline 3 & phenol sulfate & 53.01 & Tyrosine Metabolism \\
\hline 4 & tartarate & 31.9 & Food Component/Plant \\
\hline 5 & maleate & 20.29 & Fatty Acid, Dicarboxylate \\
\hline 6 & p-cresol sulfate & 17.68 & Benzoate Metabolism \\
\hline 7 & $\begin{array}{r}\text { nicotinamide adenine dinucleotide } \\
\text { (NAD+) }\end{array}$ & 16.82 & Nicotinate and Nicotinamide Metabolism \\
\hline 8 & arabinose & 16.44 & Pentose Metabolism \\
\hline 9 & benzoylcarnitine* & 15.64 & Chemical \\
\hline 10 & salicylate & 14.33 & Drug - Topical Agents \\
\hline 11 & hydroquinone sulfate & 13.42 & Drug - Topical Agents \\
\hline 12 & 3-indoxyl sulfate & 11.46 & Tryptophan Metabolism \\
\hline 13 & 2-aminoheptanoate & 10.16 & Fatty Acid, Amino \\
\hline 14 & guaiacol sulfate & 9.77 & Benzoate Metabolism \\
\hline 15 & taurolithocholate & 8.67 & Secondary Bile Acid Metabolism \\
\hline 16 & glutarate (C5-DC) & 8.47 & Fatty Acid, Dicarboxylate \\
\hline 17 & cysteine sulfinic acid & 7.67 & $\begin{array}{l}\text { Methionine, Cysteine, SAM and Taurine } \\
\text { Metabolism }\end{array}$ \\
\hline 18 & glucuronate & 4.98 & Aminosugar Metabolism \\
\hline 19 & 1-methyl-4-imidazoleacetate & 4.6 & Histidine Metabolism \\
\hline 20 & ethylmalonate & 4.21 & Leucine, Isoleucine and Valine Metabolism \\
\hline 21 & adipate (C6-DC) & 4.16 & Fatty Acid, Dicarboxylate \\
\hline 22 & cyano-alanine & 4.14 & $\begin{array}{l}\text { Methionine, Cysteine, SAM and Taurine } \\
\text { Metabolism }\end{array}$ \\
\hline 23 & lanthionine & 4.07 & $\begin{array}{l}\text { Methionine, Cysteine, SAM and Taurine } \\
\text { Metabolism }\end{array}$ \\
\hline 24 & sebacate $(C 10-D C)$ & 3.89 & Fatty Acid, Dicarboxylate \\
\hline 25 & glycerate & 3.83 & $\begin{array}{l}\text { Glycolysis, Gluconeogenesis, and Pyruvate } \\
\text { Metabolism }\end{array}$ \\
\hline 26 & methylsuccinate & 3.8 & Leucine, Isoleucine and Valine Metabolism \\
\hline 27 & palmitoleoylcarnitine $(\mathrm{C} 16: 1)^{*}$ & 3.77 & Fatty Acid Metabolism(Acyl Carnitine) \\
\hline 28 & pheophorbide A & 3.71 & Food Component/Plant \\
\hline 29 & phytosphingosine & 3.59 & Sphingolipid Synthesis \\
\hline 30 & xylose & 3.51 & Pentose Metabolism \\
\hline 31 & 2-hydroxyglutarate & 3.39 & Fatty Acid, Dicarboxylate \\
\hline 32 & 3-methylglutarate/2-methylglutarate & 3.34 & Fatty Acid, Dicarboxylate \\
\hline 33 & linoleoylcarnitine (C18:2)* & 3.26 & Fatty Acid Metabolism(Acyl Carnitine) \\
\hline 34 & 2'-deoxyinosine & 3.18 & $\begin{array}{l}\text { Purine Metabolism, (Hypo)Xanthine/Inosine } \\
\text { containing }\end{array}$ \\
\hline 35 & thymol sulfate & 3.18 & Food Component/Plant \\
\hline 36 & $\mathrm{~N}$-formylmethionine & 3.08 & $\begin{array}{l}\text { Methionine, Cysteine, SAM and Taurine } \\
\text { Metabolism }\end{array}$ \\
\hline 37 & creatine phosphate & 3.06 & Creatine Metabolism \\
\hline 38 & 4-hydroxyphenylpyruvate & 3 & Tyrosine Metabolism \\
\hline 39 & 1-methyl-5-imidazoleacetate & 2.97 & Histidine Metabolism \\
\hline 40 & glutathione, oxidized (GSSG) & 2.95 & Glutathione Metabolism \\
\hline
\end{tabular}

Metabolites in bold are also found in the top 40 when normalized to protein content. Metabolites with asterisks indicate compounds that have not been confirmed based on standards, but Metabolon is confident in their identity. 
Table 4.4. Top 40 significantly upregulated metabolites during short-term aerial exposure in post-DIl embryos normalized to DNA concentration.

\begin{tabular}{|c|c|c|c|}
\hline Rank & Significant metabolite & $\begin{array}{c}\text { Metabolite } \\
\text { Fold } \\
\text { change }\end{array}$ & Associated sub pathway \\
\hline 1 & lanthionine & 16.93 & $\begin{array}{l}\text { Methionine, Cysteine, SAM and Taurine } \\
\text { Metabolism }\end{array}$ \\
\hline 2 & N-acetyl-1-methylhistidine* & 15.39 & Histidine Metabolism \\
\hline 3 & 3-chlorotyrosine & 8.24 & Tyrosine Metabolism \\
\hline 4 & phenol sulfate & 8.15 & Tyrosine Metabolism \\
\hline 5 & pterin & 8.05 & Pterin Metabolism \\
\hline 6 & N-acetyl-3-methylhistidine* & 7.21 & Histidine Metabolism \\
\hline 7 & linolenoylcarnitine (C18:3)* & 6.65 & Fatty Acid Metabolism(Acyl Carnitine) \\
\hline 8 & picolinate & 5.1 & Tryptophan Metabolism \\
\hline 9 & allantoin & 4.67 & $\begin{array}{l}\text { Purine Metabolism, (Hypo)Xanthine/Inosine } \\
\text { containing }\end{array}$ \\
\hline 10 & xanthopterin & 4.03 & Pterin Metabolism \\
\hline 11 & taurochenodeoxycholate & 3.74 & Primary Bile Acid Metabolism \\
\hline 12 & 2'-O-methyluridine & 3.56 & Pyrimidine Metabolism, Uracil containing \\
\hline 13 & kynurenate & 3.31 & Tryptophan Metabolism \\
\hline 14 & guanine & 3.16 & Purine Metabolism, Guanine containing \\
\hline 15 & pro-hydroxy-pro & 3.09 & Urea cycle; Arginine and Proline Metabolism \\
\hline 16 & methionine sulfone & 2.97 & $\begin{array}{l}\text { Methionine, Cysteine, SAM and Taurine } \\
\text { Metabolism }\end{array}$ \\
\hline 17 & arachidonoylcarnitine (C20:4) & 2.94 & Fatty Acid Metabolism(Acyl Carnitine) \\
\hline 18 & N2-acetyllysine & 2.8 & Lysine Metabolism \\
\hline 19 & mannose-6-phosphate & 2.72 & Fructose, Mannose and Galactose Metabolism \\
\hline 20 & $\begin{array}{r}\text { adenosine 3'-monophosphate } \\
\text { (3'-AMP) }\end{array}$ & 2.69 & Purine Metabolism, Adenine containing \\
\hline 21 & glucose 6-phosphate & 2.68 & $\begin{array}{l}\text { Glycolysis, Gluconeogenesis, and Pyruvate } \\
\text { Metabolism }\end{array}$ \\
\hline 22 & $\begin{array}{r}\text { methyl glucopyranoside } \\
\text { (alpha + beta) }\end{array}$ & 2.65 & Food Component/Plant \\
\hline 23 & 2'-deoxyinosine & 2.6 & $\begin{array}{l}\text { Purine Metabolism, (Hypo)Xanthine/Inosine } \\
\text { containing }\end{array}$ \\
\hline 24 & $\begin{array}{l}\text { docosapentaenoylcarnitine } \\
(\text { (C22:5n6)* }\end{array}$ & 2.6 & Fatty Acid Metabolism(Acyl Carnitine) \\
\hline 25 & $\begin{array}{r}\text { uridine 2'-monophosphate } \\
(2 '-U M P)^{*}\end{array}$ & 2.56 & Pyrimidine Metabolism, Uracil containing \\
\hline 26 & palmitoleoylcarnitine (C16:1)* & 2.36 & Fatty Acid Metabolism(Acyl Carnitine) \\
\hline 27 & glutathione, oxidized (GSSG) & 2.24 & Glutathione Metabolism \\
\hline 28 & ceramide (d16:1/24:1, d18:1/22:1)* & 2.12 & Ceramides \\
\hline 29 & uridine & 2.12 & Pyrimidine Metabolism, Uracil containing \\
\hline 30 & betaine aldehyde & 2.1 & Glycine, Serine and Threonine Metabolism \\
\hline 31 & myristoylcarnitine (C14) & 2.1 & Fatty Acid Metabolism(Acyl Carnitine) \\
\hline 32 & linoleoylcarnitine (C18:2)* & 2.1 & Fatty Acid Metabolism(Acyl Carnitine) \\
\hline 33 & sedoheptulose-7-phosphate & 1.98 & Pentose Phosphate Pathway \\
\hline 34 & cystathionine & 1.89 & $\begin{array}{l}\text { Methionine, Cysteine, SAM and Taurine } \\
\text { Metabolism }\end{array}$ \\
\hline 35 & alpha-ketobutyrate & 1.64 & $\begin{array}{l}\text { Methionine, Cysteine, SAM and Taurine } \\
\text { Metabolism }\end{array}$ \\
\hline 36 & phosphoenolpyruvate (PEP) & 1.64 & $\begin{array}{l}\text { Glycolysis, Gluconeogenesis, and Pyruvate } \\
\text { Metabolism }\end{array}$ \\
\hline 37 & cyano-alanine & 1.62 & $\begin{array}{l}\text { Methionine, Cysteine, SAM and Taurine } \\
\text { Metabolism }\end{array}$ \\
\hline 38 & galactose 1-phosphate & 1.62 & Fructose, Mannose and Galactose Metabolism \\
\hline 39 & 4-acetamidobutanoate & 1.59 & Polyamine Metabolism \\
\hline 40 & (3'-5')-adenylyluridine & 1.58 & Dinucleotide \\
\hline
\end{tabular}

Metabolites in bold are also found in the top 40 when normalized to protein content. Metabolites with asterisks indicate compounds that have not been confirmed based on standards, but Metabolon is confident in their identity. 
Table 4.5. Top 40 significantly upregulated metabolites during long-term aerial exposure in post-DII embryos normalized to DNA concentration.

\begin{tabular}{|c|c|c|c|}
\hline Rank & Significant metabolite & $\begin{array}{l}\text { Metabolite } \\
\text { Fold } \\
\text { change }\end{array}$ & Associated sub pathway \\
\hline 1 & lanthionine & 868.97 & $\begin{array}{l}\text { Methionine, Cysteine, SAM and Taurine } \\
\text { Metabolism }\end{array}$ \\
\hline 2 & methyl glucopyranoside (alpha + beta) & 207.74 & Food Component/Plant \\
\hline 3 & xanthopterin & 133.64 & Pterin Metabolism \\
\hline 4 & taurochenodeoxycholate & 132.19 & Primary Bile Acid Metabolism \\
\hline 5 & indoleacetylcarnitine $^{*}$ & 98.21 & Chemical \\
\hline 6 & kynurenate & 80.86 & Tryptophan Metabolism \\
\hline 7 & N-acetyl-1-methylhistidine* & 75.31 & Histidine Metabolism \\
\hline 8 & adenosine 2'-monophosphate (2'-AMP) & 68.14 & Purine Metabolism, Adenine containing \\
\hline 9 & phenol sulfate & 58.17 & Tyrosine Metabolism \\
\hline 10 & biopterin & 57.99 & Tetrahydrobiopterin Metabolism \\
\hline 11 & sedoheptulose & 56.67 & Pentose Metabolism \\
\hline 12 & taurocholate & 56.18 & Primary Bile Acid Metabolism \\
\hline 13 & N2-acetyllysine & 52.59 & Lysine Metabolism \\
\hline 14 & dihydrobiopterin & 50.04 & Tetrahydrobiopterin Metabolism \\
\hline 15 & $\mathrm{~N}$-acetyl-3-methylhistidine* & 46.95 & Histidine Metabolism \\
\hline 16 & $(N(1)+N(8))$-acetylspermidine & 41.13 & Polyamine Metabolism \\
\hline 17 & allantoin & 39.12 & $\begin{array}{l}\text { Purine Metabolism, (Hypo)Xanthine/Inosine } \\
\text { containing }\end{array}$ \\
\hline 18 & cytidine 5'-diphosphocholine & 35.47 & Phospholipid Metabolism \\
\hline 19 & 7-methylguanine & 33.21 & Purine Metabolism, Guanine containing \\
\hline 20 & glutathione, oxidized (GSSG) & 31.19 & Glutathione Metabolism \\
\hline 21 & guanosine 2'-monophosphate (2'-GMP)* & 29.95 & Purine Metabolism, Guanine containing \\
\hline 22 & 3-chlorotyrosine & 29.12 & Tyrosine Metabolism \\
\hline 23 & picolinate & 28.53 & Tryptophan Metabolism \\
\hline 24 & alpha-ketobutyrate & 27 & $\begin{array}{l}\text { Methionine, Cysteine, SAM and Taurine } \\
\text { Metabolism }\end{array}$ \\
\hline 25 & ribose & 26.65 & Pentose Metabolism \\
\hline 26 & cystathionine & 25.67 & $\begin{array}{l}\text { Methionine, Cysteine, SAM and Taurine } \\
\text { Metabolism }\end{array}$ \\
\hline 27 & isoxanthopterin & 23.02 & Pterin Metabolism \\
\hline 28 & urate & 21.73 & $\begin{array}{l}\text { Purine Metabolism, (Hypo)Xanthine/Inosine } \\
\text { containing }\end{array}$ \\
\hline 29 & hypoxanthine & 21.39 & $\begin{array}{l}\text { Purine Metabolism, (Hypo)Xanthine/Inosine } \\
\text { containing }\end{array}$ \\
\hline 30 & gamma-aminobutyrate (GABA) & 20.33 & Glutamate Metabolism \\
\hline 31 & uracil & 18.77 & Pyrimidine Metabolism, Uracil containing \\
\hline 32 & uridine 2'-monophosphate (2'-UMP)* & 18.57 & Pyrimidine Metabolism, Uracil containing \\
\hline 33 & $\mathrm{~N}$-acetylglucosamine 6-phosphate & 18.18 & Aminosugar Metabolism \\
\hline 34 & argininate* $^{*}$ & 18.13 & Urea cycle; Arginine and Proline Metabolism \\
\hline 35 & ceramide $(\mathrm{d} 16: 1 / 24: 1, \mathrm{~d} 18: 1 / 22: 1)^{*}$ & 17.76 & Ceramides \\
\hline 36 & $\begin{array}{r}\text { 1-(1-enyl-palmitoyl)-2-linoleoyl-GPE (P- } \\
16: 0 / 18: 2)^{*}\end{array}$ & 17.12 & Plasmalogen \\
\hline 37 & pterin & 17.05 & Pterin Metabolism \\
\hline 38 & sedoheptulose-7-phosphate & 16.82 & Pentose Phosphate Pathway \\
\hline 39 & adenosine 3'-monophosphate (3'-AMP) & 16.29 & Purine Metabolism, Adenine containing \\
\hline 40 & betaine aldehyde & 14.92 & Glycine, Serine and Threonine Metabolism \\
\hline
\end{tabular}

Metabolites in bold are also found in the top 40 when normalized to protein content. Metabolites with asterisks indicate compounds that have not been confirmed based on standards, but Metabolon is confident in their identity. 
Table 4.6. Top 30 significantly downregulated metabolites during short-term aerial exposure in DII embryos normalized to DNA concentration.

\begin{tabular}{|c|c|c|c|}
\hline Rank & Significant metabolite & $\begin{array}{c}\text { Metabolite } \\
\text { Fold } \\
\text { change }\end{array}$ & Associated sub pathway \\
\hline 1 & nicotinate & 0.01 & Nicotinate and Nicotinamide Metabolism \\
\hline 2 & ergothioneine & 0.03 & Food Component/Plant \\
\hline 3 & hypotaurine & 0.08 & $\begin{array}{l}\text { Methionine, Cysteine, SAM and Taurine } \\
\text { Metabolism }\end{array}$ \\
\hline 4 & $\mathrm{~N}$-acetylputrescine & 0.16 & Polyamine Metabolism \\
\hline 5 & pheophytin A & 0.18 & Food Component/Plant \\
\hline 6 & nicotinamide ribonucleotide (NMN) & 0.19 & Nicotinate and Nicotinamide Metabolism \\
\hline 7 & 1-docosahexaenoylglycerol (22:6) & 0.21 & Monoacylglycerol \\
\hline 8 & 2-docosahexaenoylglycerol $(22: 6)^{*}$ & 0.22 & Monoacylglycerol \\
\hline 9 & N-carbamoylaspartate & 0.24 & Pyrimidine Metabolism, Orotate containing \\
\hline 10 & hexadecatrienoate $(16: 3 n 3)$ & 0.27 & Polyunsaturated Fatty Acid (n3 and n6) \\
\hline 11 & 1-myristoylglycerol (14:0) & 0.28 & Monoacylglycerol \\
\hline 12 & 1-palmitoleoylglycerol $(16: 1)^{*}$ & 0.28 & Monoacylglycerol \\
\hline 13 & tetradecadienoate $(14: 2)^{*}$ & 0.28 & Polyunsaturated Fatty Acid (n3 and n6) \\
\hline 14 & 1-heptadecenoylglycerol $(17: 1)^{*}$ & 0.28 & Monoacylglycerol \\
\hline 15 & 1-linoleoylglycerol (18:2) & 0.29 & Monoacylglycerol \\
\hline 16 & 1-dihomo-linolenylglycerol (20:3) & 0.3 & Monoacylglycerol \\
\hline 17 & myristoleate (14:1n5) & 0.31 & Long Chain Fatty Acid \\
\hline 18 & trans-urocanate & 0.34 & Histidine Metabolism \\
\hline 19 & 2,3-dihydroxyisovalerate & 0.36 & Food Component/Plant \\
\hline 20 & xanthine & 0.39 & $\begin{array}{l}\text { Purine Metabolism, (Hypo)Xanthine/Inosine } \\
\text { containing }\end{array}$ \\
\hline 21 & 4-hydroxy-nonenal-glutathione & 0.41 & Glutathione Metabolism \\
\hline 22 & sucrose & 0.46 & Disaccharides and Oligosaccharides \\
\hline 23 & hexadecadienoate $(16: 2 n 6)$ & 0.47 & Polyunsaturated Fatty Acid (n3 and n6) \\
\hline 24 & adenosine & 0.52 & Purine Metabolism, Adenine containing \\
\hline 25 & N-acetylmethionine & 0.54 & $\begin{array}{l}\text { Methionine, Cysteine, SAM and Taurine } \\
\text { Metabolism }\end{array}$ \\
\hline 26 & adenine & 0.54 & Purine Metabolism, Adenine containing \\
\hline 27 & guanine & 0.55 & Purine Metabolism, Guanine containing \\
\hline 28 & dihomo-linoleoylcarnitine $(\mathrm{C} 20: 2)^{*}$ & 0.57 & Fatty Acid Metabolism(Acyl Carnitine) \\
\hline 29 & dihydroxyacetone phosphate (DHAP) & 0.57 & $\begin{array}{l}\text { Glycolysis, Gluconeogenesis, and Pyruvate } \\
\text { Metabolism }\end{array}$ \\
\hline 30 & uracil & 0.61 & Pyrimidine Metabolism, Uracil containing \\
\hline
\end{tabular}

Metabolites in bold are also found in the top 40 when normalized to protein content. Metabolites with asterisks indicate compounds that have not been confirmed based on standards, but Metabolon is confident in their identity. 
Table 4.7. Top 34 significantly downregulated metabolites during long-term aerial exposure in DII embryos normalized to DNA concentration.

\begin{tabular}{|c|c|c|c|}
\hline Rank & Significant metabolite & $\begin{array}{c}\text { Metabolite } \\
\text { Fold } \\
\text { change }\end{array}$ & Associated sub pathway \\
\hline 1 & nicotinate & 0.02 & Nicotinate and Nicotinamide Metabolism \\
\hline 2 & ergothioneine & 0.06 & Food Component/Plant \\
\hline 3 & nicotinamide ribonucleotide (NMN) & 0.15 & Nicotinate and Nicotinamide Metabolism \\
\hline 4 & $\mathrm{~N}$-acetylputrescine & 0.16 & Polyamine Metabolism \\
\hline 5 & sucrose & 0.16 & Disaccharides and Oligosaccharides \\
\hline 6 & 1-heptadecenoylglycerol $(17: 1)^{*}$ & 0.17 & Monoacylglycerol \\
\hline 7 & 1-docosahexaenoylglycerol $(22: 6)$ & 0.21 & Monoacylglycerol \\
\hline 8 & guanine & 0.22 & Purine Metabolism, Guanine containing \\
\hline 9 & tetradecadienoate $(14: 2)^{*}$ & 0.25 & Polyunsaturated Fatty Acid (n3 and n6) \\
\hline 10 & 1-oleoylglycerol (18:1) & 0.25 & Monoacylglycerol \\
\hline 11 & 1-myristoylglycerol (14:0) & 0.26 & Monoacylglycerol \\
\hline 12 & 1-palmitoleoylglycerol $(16: 1)^{*}$ & 0.26 & Monoacylglycerol \\
\hline 13 & pheophytin A & 0.27 & Food Component/Plant \\
\hline 14 & hexadecatrienoate $(16: 3 n 3)$ & 0.27 & Polyunsaturated Fatty Acid (n3 and n6) \\
\hline 15 & 1-dihomo-linolenylglycerol (20:3) & 0.27 & Monoacylglycerol \\
\hline 16 & 1-linoleoylglycerol (18:2) & 0.28 & Monoacylglycerol \\
\hline 17 & 2-heptadecenoylglycerol $(17: 1)^{*}$ & 0.29 & Monoacylglycerol \\
\hline 18 & myristoleate $(14: 1 \mathrm{n} 5)$ & 0.3 & Long Chain Fatty Acid \\
\hline 19 & 2-docosahexaenoylglycerol $(22: 6)^{*}$ & 0.31 & Monoacylglycerol \\
\hline 20 & hypoxanthine & 0.31 & $\begin{array}{l}\text { Purine Metabolism, (Hypo)Xanthine/Inosine } \\
\text { containing }\end{array}$ \\
\hline 21 & N-carbamoylaspartate & 0.34 & Pyrimidine Metabolism, Orotate containing \\
\hline 22 & trans-urocanate & 0.35 & Histidine Metabolism \\
\hline 23 & adenosine & 0.36 & Purine Metabolism, Adenine containing \\
\hline 24 & hexadecadienoate $(16: 2 \mathrm{n} 6)$ & 0.37 & Polyunsaturated Fatty Acid (n3 and n6) \\
\hline 25 & 4-hydroxy-nonenal-glutathione & 0.41 & Glutathione Metabolism \\
\hline 26 & 13-HODE + 9-HODE & 0.44 & Fatty Acid, Monohydroxy \\
\hline 27 & 3-hydroxybutyrate (BHBA) & 0.45 & Ketone Bodies \\
\hline 28 & 2-palmitoleoylglycerol $(16: 1)^{*}$ & 0.46 & Monoacylglycerol \\
\hline 29 & ophthalmate & 0.48 & Glutathione Metabolism \\
\hline 30 & xanthine & 0.52 & $\begin{array}{l}\text { Purine Metabolism, (Hypo)Xanthine/Inosine } \\
\text { containing }\end{array}$ \\
\hline 31 & adenine & 0.53 & Purine Metabolism, Adenine containing \\
\hline 32 & dihydroxyacetone phosphate (DHAP) & 0.57 & $\begin{array}{l}\text { Glycolysis, Gluconeogenesis, and Pyruvate } \\
\text { Metabolism }\end{array}$ \\
\hline 33 & uracil & 0.58 & Pyrimidine Metabolism, Uracil containing \\
\hline 34 & 1-arachidonylglycerol $(20: 4)$ & 0.61 & Monoacylglycerol \\
\hline
\end{tabular}

Metabolites in bold are also found in the top 40 when normalized to protein content. Metabolites with asterisks indicate compounds that have not been confirmed based on standards, but Metabolon is confident in their identity. 
Table 4.8. Top 40 significantly downregulated metabolites during short-term aerial exposure in post-DII embryos normalized to DNA concentration.

\begin{tabular}{|c|c|c|c|}
\hline Rank & Significant metabolite & $\begin{array}{l}\text { Metabolite } \\
\text { Fold } \\
\text { change }\end{array}$ & Associated sub pathway \\
\hline 1 & nicotinate & 0.01 & Nicotinate and Nicotinamide Metabolism \\
\hline 2 & adenosine 5'-monophosphate (AMP) & 0.01 & Purine Metabolism, Adenine containing \\
\hline 3 & $\begin{array}{r}\text { 2'-deoxyguanosine 5'-monophosphate } \\
\text { (dGMP) }\end{array}$ & 0.02 & Purine Metabolism, Guanine containing \\
\hline 4 & 5-oxoproline & 0.03 & Glutathione Metabolism \\
\hline 5 & trans-urocanate & 0.06 & Histidine Metabolism \\
\hline 6 & pipecolate & 0.06 & Lysine Metabolism \\
\hline 7 & creatine phosphate & 0.06 & Creatine Metabolism \\
\hline 8 & gamma-glutamylalanine & 0.06 & Gamma-glutamyl Amino Acid \\
\hline 9 & gamma-glutamyl-2-aminobutyrate & 0.07 & Gamma-glutamyl Amino Acid \\
\hline 10 & ergothioneine & 0.08 & \multirow{2}{*}{$\begin{array}{l}\text { Food Component/Plant } \\
\text { Methionine, Cysteine, SAM and Taurine } \\
\text { Metabolism }\end{array}$} \\
\hline 11 & cystine & 0.08 & \\
\hline 12 & sucrose & 0.08 & Disaccharides and Oligosaccharides \\
\hline 13 & 3-ureidopropionate & 0.09 & Pyrimidine Metabolism, Uracil containing \\
\hline 14 & 2'-deoxyadenosine 5'-monophosphate & 0.09 & Purine Metabolism, Adenine containing \\
\hline 15 & $\mathrm{~N}$-acetylputrescine & 0.1 & Polyamine Metabolism \\
\hline 16 & 2-oxoarginine* & 0.1 & \multirow{2}{*}{$\begin{array}{l}\text { Urea cycle; Arginine and Proline Metabolism } \\
\text { Methionine, Cysteine, SAM and Taurine } \\
\text { Metabolism }\end{array}$} \\
\hline 17 & hypotaurine & 0.11 & \\
\hline 18 & $\mathrm{~N}$-acetylneuraminate & 0.11 & \multirow{2}{*}{$\begin{array}{l}\text { Aminosugar Metabolism } \\
\text { Purine Metabolism, (Hypo)Xanthine/lnosine } \\
\text { containing }\end{array}$} \\
\hline 19 & inosine 5'-monophosphate (IMP) & 0.11 & \\
\hline 20 & lysylleucine & 0.12 & Dipeptide \\
\hline 21 & nicotinamide ribonucleotide (NMN) & 0.12 & Nicotinate and Nicotinamide Metabolism \\
\hline 22 & thymidine 5 '-monophosphate & 0.13 & Pyrimidine Metabolism, Thymine containing \\
\hline 23 & 4-imidazoleacetate & 0.13 & Histidine Metabolism \\
\hline 24 & mannose & 0.13 & Fructose, Mannose and Galactose Metabolism \\
\hline 25 & 2'-deoxyadenosine & 0.13 & Purine Metabolism, Adenine containing \\
\hline 26 & $\begin{array}{r}\text { linoleoyl-arachidonoyl-glycerol } \\
(18: 2 / 20: 4)[1]^{*}\end{array}$ & 0.14 & Diacylglycerol \\
\hline 27 & serine & 0.14 & Glycine, Serine and Threonine Metabolism \\
\hline 28 & isocitrate & 0.14 & TCA Cycle \\
\hline 29 & asparagine & 0.15 & Alanine and Aspartate Metabolism \\
\hline 30 & aconitate [cis or trans] & 0.15 & TCA Cycle \\
\hline 31 & $\mathrm{~N}$-carbamoylaspartate & 0.16 & Pyrimidine Metabolism, Orotate containing \\
\hline 32 & isoleucylglycine & 0.16 & Dipeptide \\
\hline 33 & 1-palmitoleoylglycerol $(16: 1)^{*}$ & 0.16 & Monoacylglycerol \\
\hline 34 & 2'-deoxyuridine & 0.17 & Pyrimidine Metabolism, Uracil containing \\
\hline 35 & linoleoylcholine* & 0.17 & \multirow{2}{*}{$\begin{array}{l}\text { Fatty Acid Metabolism (Acyl Choline) } \\
\text { Methionine, Cysteine, SAM and Taurine } \\
\text { Metabolism }\end{array}$} \\
\hline 36 & cysteine sulfinic acid & 0.18 & \\
\hline 37 & alanine & 0.18 & Alanine and Aspartate Metabolism \\
\hline 38 & 2'-deoxycytidine & 0.18 & Pyrimidine Metabolism, Cytidine containing \\
\hline 39 & glutamate & 0.18 & Glutamate Metabolism \\
\hline 40 & pyridoxamine & 0.18 & Vitamin B6 Metabolism \\
\hline
\end{tabular}

Metabolites in bold are also found in the top 40 when normalized to protein content. Metabolites with asterisks indicate compounds that have not been confirmed based on standards, but Metabolon is confident in their identity. 
Table 4.9. Top 40 significantly downregulated metabolites during long-term aerial exposure in post-DIl embryos normalized to DNA concentration.

\begin{tabular}{|c|c|c|c|}
\hline Rank & Significant metabolite & $\begin{array}{l}\text { Metabolite } \\
\text { Fold } \\
\text { change }\end{array}$ & Associated sub pathway \\
\hline 1 & gamma-glutamyl-2-aminobutyrate & 0.01 & Gamma-glutamyl Amino Acid \\
\hline 2 & inosine 5'-monophosphate (IMP) & 0.01 & $\begin{array}{l}\text { Purine Metabolism, (Hypo)Xanthine/Inosine } \\
\text { containing }\end{array}$ \\
\hline 3 & gamma-glutamylglutamate & 0.01 & Gamma-glutamyl Amino Acid \\
\hline 4 & 5-oxoproline & 0.03 & Glutathione Metabolism \\
\hline 5 & gamma-glutamylalanine & 0.03 & Gamma-glutamyl Amino Acid \\
\hline 6 & adenosine 5'-monophosphate (AMP) & 0.04 & Purine Metabolism, Adenine containing \\
\hline 7 & creatine phosphate & 0.04 & Creatine Metabolism \\
\hline 8 & gamma-glutamylglycine & 0.04 & Gamma-glutamyl Amino Acid \\
\hline 9 & isocitrate & 0.05 & TCA Cycle \\
\hline 10 & gamma-glutamylserine & 0.05 & Gamma-glutamyl Amino Acid \\
\hline 11 & oleoylcholine & 0.06 & Fatty Acid Metabolism (Acyl Choline) \\
\hline 12 & uridine 5'-monophosphate (UMP) & 0.06 & Pyrimidine Metabolism, Uracil containing \\
\hline 13 & sucrose & 0.07 & Disaccharides and Oligosaccharides \\
\hline 14 & aconitate [cis or trans] & 0.07 & TCA Cycle \\
\hline 15 & nicotinate & 0.08 & Nicotinate and Nicotinamide Metabolism \\
\hline 16 & 5,6-dihydrouridine & 0.08 & Pyrimidine Metabolism, Uracil containing \\
\hline 17 & 2'-deoxyadenosine & 0.09 & Purine Metabolism, Adenine containing \\
\hline 18 & $\mathrm{~N}$-acetylneuraminate & 0.1 & Aminosugar Metabolism \\
\hline 19 & maltotetraose & 0.11 & Glycogen Metabolism \\
\hline 20 & pipecolate & 0.12 & Lysine Metabolism \\
\hline 21 & 2-palmitoleoylglycerol $(16: 1)^{*}$ & 0.12 & Monoacylglycerol \\
\hline 22 & guanidinoacetate & 0.13 & Creatine Metabolism \\
\hline 23 & linoleoylcholine* & 0.14 & Fatty Acid Metabolism (Acyl Choline) \\
\hline 24 & citrate & 0.14 & TCA Cycle \\
\hline 25 & palmitoloelycholine & 0.15 & Fatty Acid Metabolism (Acyl Choline) \\
\hline 26 & 2-arachidonoylglycerol (20:4) & 0.15 & Monoacylglycerol \\
\hline 27 & palmitoylcholine & 0.16 & Fatty Acid Metabolism (Acyl Choline) \\
\hline 28 & 1-arachidonylglycerol (20:4) & 0.16 & Monoacylglycerol \\
\hline 29 & adenosine & 0.16 & Purine Metabolism, Adenine containing \\
\hline 30 & stearoylcholine* $^{*}$ & 0.17 & Fatty Acid Metabolism (Acyl Choline) \\
\hline 31 & gamma-glutamylthreonine & 0.17 & Gamma-glutamyl Amino Acid \\
\hline 32 & 3-aminoisobutyrate & 0.17 & Pyrimidine Metabolism, Thymine containing \\
\hline 33 & 1-palmitoleoylglycerol $(16: 1)^{*}$ & 0.18 & Monoacylglycerol \\
\hline 34 & $\begin{array}{l}\text { 2'-deoxyguanosine 5'- } \\
\text { monophosphate (dGMP) }\end{array}$ & 0.19 & Purine Metabolism, Guanine containing \\
\hline 35 & maltopentaose & 0.19 & Glycogen Metabolism \\
\hline 36 & $\begin{array}{l}\mathrm{N} \text {-acetylglucosamine/N- } \\
\text { acetylgalactosamine }\end{array}$ & 0.19 & Aminosugar Metabolism \\
\hline 37 & oleoyl-oleoyl-glycerol (18:1/18:1) [1] ${ }^{*}$ & 0.19 & Diacylglycerol \\
\hline 38 & 4-hydroxyglutamate & 0.2 & Glutamate Metabolism \\
\hline 39 & 5-methyluridine (ribothymidine) & 0.21 & Pyrimidine Metabolism, Uracil containing \\
\hline 40 & $\mathrm{~N}$-acetyl-aspartyl-glutamate (NAAG) & 0.21 & Glutamate Metabolism \\
\hline
\end{tabular}


Table 4.10. Metabolic pathways that were significantly enriched by short and long-term aerial exposure in DII and post-DII embryos normalized to DNA concentration.

\begin{tabular}{|c|c|c|c|c|c|}
\hline $\begin{array}{l}\text { Treatment } \\
\text { (\# significant } \\
\text { metabolites) }\end{array}$ & Subpathway & $\begin{array}{l}\text { Enrichment } \\
\text { Value }\end{array}$ & P-value & k & $\mathbf{m}$ \\
\hline \multirow{7}{*}{$\begin{array}{c}\text { DII } \\
\text { short-term } \\
(n=204)\end{array}$} & Fatty Acid, Dihydroxy & 3.3 & 0.03 & 3 & 3 \\
\hline & Fatty Acid, Dicarboxylate & 2.6 & $6.34 \mathrm{E}-04$ & 10 & 13 \\
\hline & Fatty Acid, Monohydroxy & 2.4 & 0.03 & 5 & 7 \\
\hline & $\begin{array}{r}\text { Nicotinate and Nicotinamide } \\
\text { Metabolism }\end{array}$ & 2.4 & 0.03 & 5 & 7 \\
\hline & Tyrosine Metabolism & 2.4 & 0.01 & 7 & 10 \\
\hline & $\begin{array}{r}\text { Methionine, Cysteine, SAM and } \\
\text { Taurine Metabolism }\end{array}$ & 2.0 & 4.17E-03 & 13 & 22 \\
\hline & $\begin{array}{r}\text { Leucine, Isoleucine and Valine } \\
\text { Metabolism }\end{array}$ & 1.9 & 0.02 & 11 & 20 \\
\hline \multirow{4}{*}{$\begin{array}{c}\text { DII } \\
\text { long-term } \\
(n=267)\end{array}$} & $\begin{array}{r}\text { Fatty Acid Metabolism (Acyl } \\
\text { Carnitine, Long Chain Saturated) }\end{array}$ & 1.9 & 0.05 & 6 & 8 \\
\hline & Tyrosine Metabolism & 1.8 & 0.05 & 7 & 10 \\
\hline & Monoacylglycerol & 1.7 & 0.03 & 11 & 17 \\
\hline & $\begin{array}{r}\text { Methionine, Cysteine, SAM and } \\
\text { Taurine Metabolism }\end{array}$ & 1.6 & 0.02 & 14 & 22 \\
\hline \multirow{5}{*}{$\begin{array}{l}\text { Post-DII } \\
\text { short-term } \\
(n=553)\end{array}$} & Phosphatidylcholine (PC) & 1.2 & 0.02 & 19 & 19 \\
\hline & $\begin{array}{r}\text { Urea cycle; Arginine and Proline } \\
\text { Metabolism }\end{array}$ & 1.2 & 0.02 & 19 & 19 \\
\hline & Gamma-glutamyl Amino Acid & 1.2 & 0.03 & 17 & 17 \\
\hline & Monoacylglycerol & 1.2 & 0.03 & 17 & 17 \\
\hline & Diacylglycerol & 1.2 & 7.71E-03 & 34 & 35 \\
\hline \multirow{5}{*}{$\begin{array}{l}\text { Post-DII } \\
\text { long-term } \\
(\mathrm{n}=390)\end{array}$} & Aminosugar Metabolism & 1.7 & 0.01 & 8 & 8 \\
\hline & $\begin{array}{r}\text { Purine Metabolism, Adenine } \\
\text { containing }\end{array}$ & 1.6 & 0.03 & 9 & 10 \\
\hline & Gamma-glutamyl Amino Acid & 1.5 & 7.36E-03 & 15 & 17 \\
\hline & Monoacylglycerol & 1.5 & 7.36E-03 & 15 & 17 \\
\hline & Phosphatidylcholine (PC) & 1.5 & 0.01 & 16 & 19 \\
\hline
\end{tabular}




\section{Dehydration exposure}

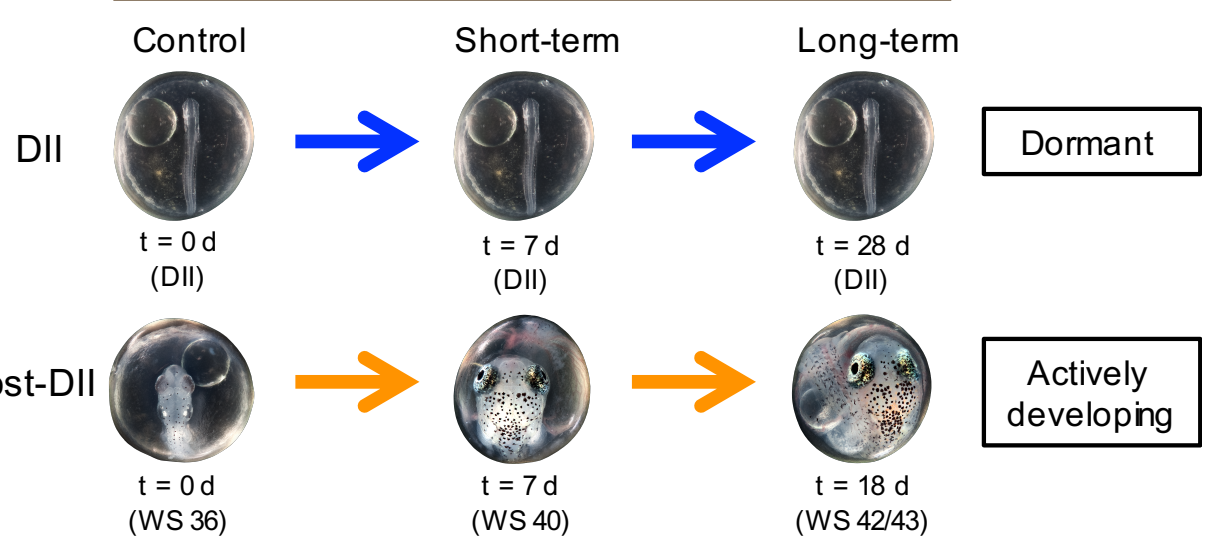

Figure 4.1. Schematic of the experimental design and sampling regimen used for metabolomics analysis. Embryos were exposed to $85 \%$ relative humidity $(\mathrm{RH})$ at the developmental stage listed (DII or WS 36). Embryos were sampled after short-term (7 d) and long-term (18 or $28 \mathrm{~d}$ ) aerial exposure ( $N=6$, each replicate contained 25 embryos).

Developmental stage at time of sampling is included below each timepoint. DII embryos sampled remained in diapause, whereas WS 36 embryos continued developing. WS, Wourms' stage; DII, diapause II. 

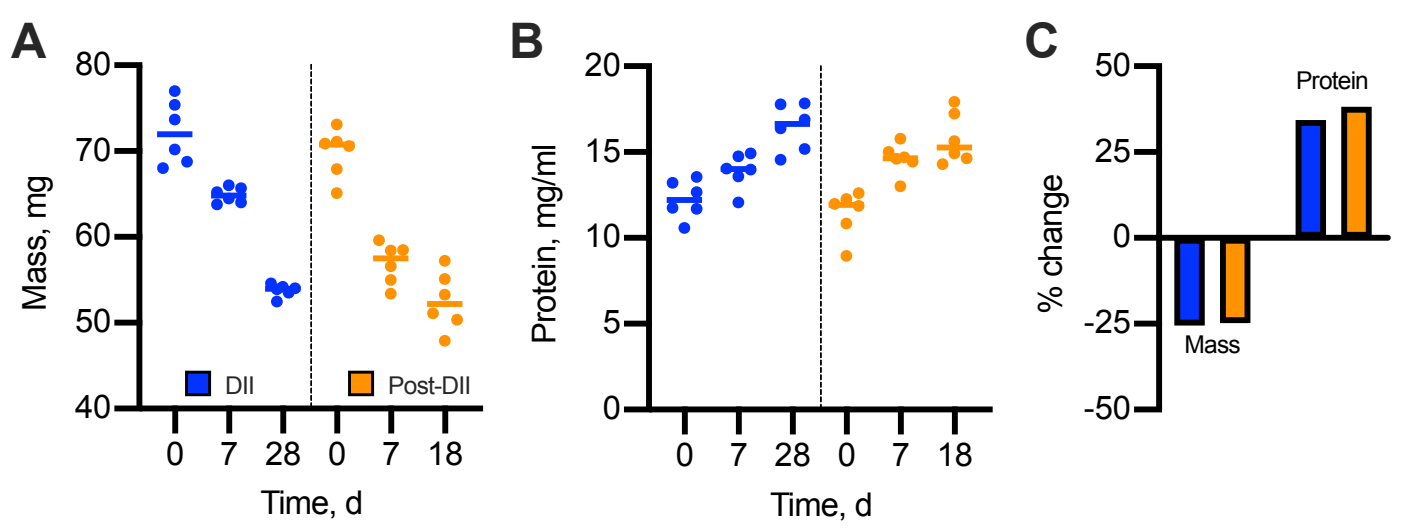

Figure 4.2. The relationship of aerial exposure on mass and protein concentration in DII and post-DIl embryos. There is a $25 \%$ decrease in mass of embryos over the course of aerial incubation in DII and post-DII embryos. Bradford protein content increases by 34 and $38 \%$ over the course of aerial incubation in DII and post-DIl embryos, respectively. Due to these systematic changes driven by water loss over the course of aerial exposure, normalization to protein exaggerates fold changes. 


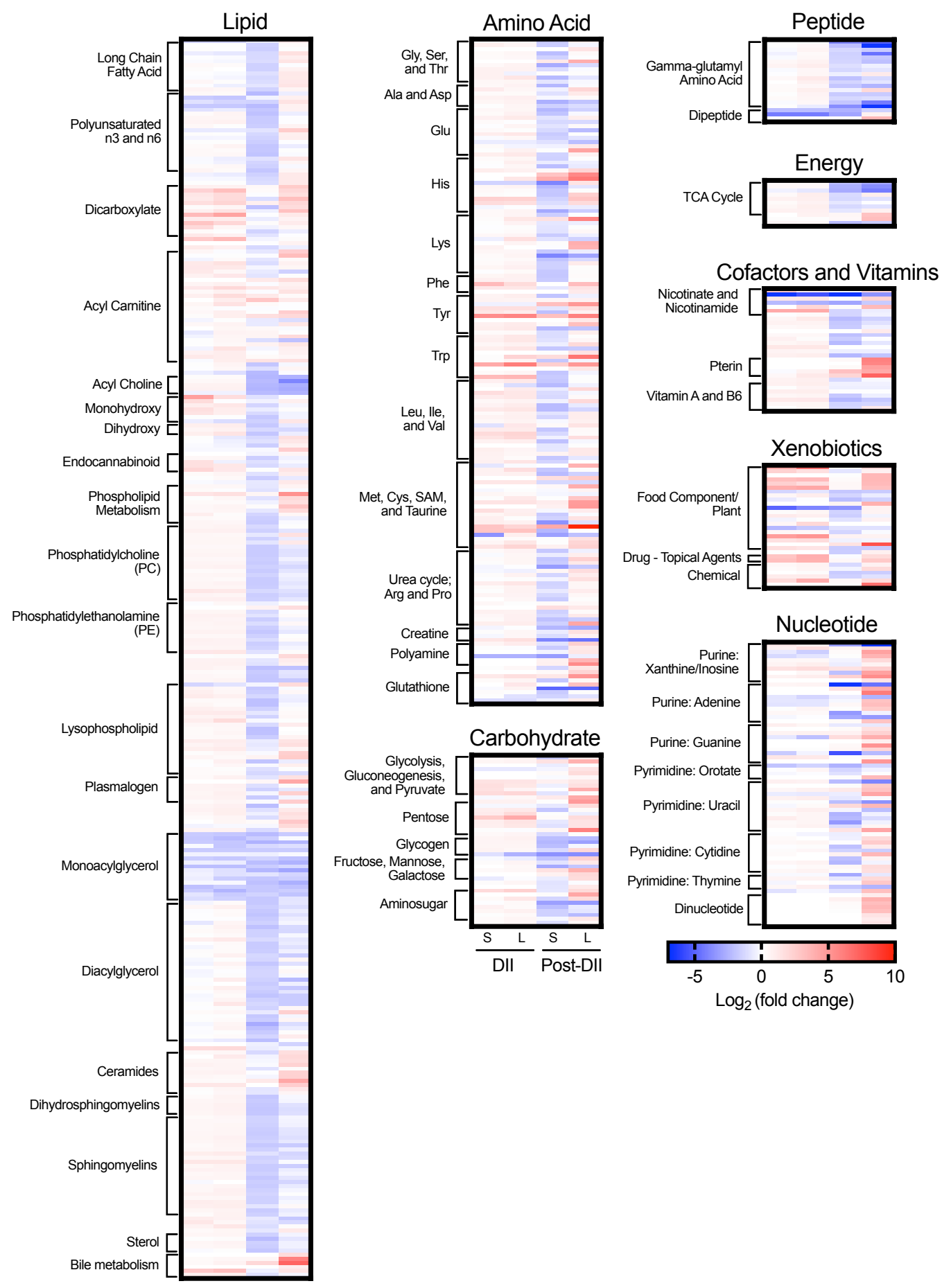

Figure 4.3. Heat maps of fold change from $t=0$ of 673 metabolites detected in embryos. Heat maps (organized by superpathway) represent the relative $\log _{2}$ transformed fold change of each metabolite in response to short-term (S) and long-term (L) aerial incubation in diapause II (DII) and post-DII embryos when compared to hydrated control embryos at $t=0$. Metabolites are clustered by subpathways. Major subpathways are indicated on the left of each heat map. Within each heatmap, fold changes are displayed for all comparisons, even though fold changes may not be statistically significant for all columns. For details on sampling, see Figure 4.1. 

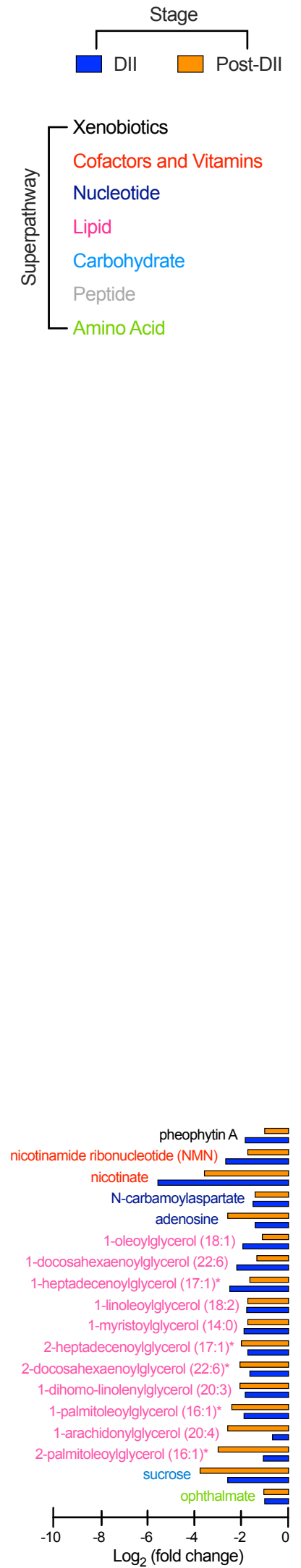

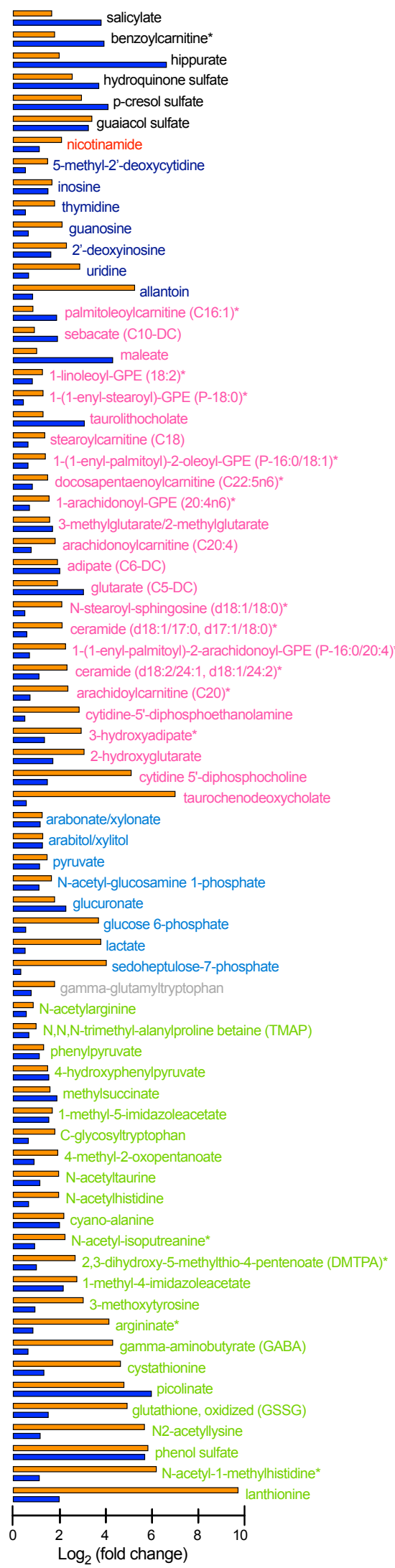

Figure 4.4. Metabolites significantly down or upregulated during longterm aerial exposure in DII and post-DII embryos. The $\log _{2}$ transformed fold changes of shared metabolites in response to long-term aerial exposure and dehydration stress when compared to hydrated control embryos at $\mathrm{t}=0$. The vertical dotted line separates the downregulated (left) and upregulated (right) metabolites. The color of each metabolite coordinates to the superpathway they belong to (above). A total of 18 metabolites decreased and 71 metabolites increased in both stages. Metabolites with asterisks indicate compounds that have not been confirmed based on standards, but Metabolon is confident in their identity. 
DII

Post-DII
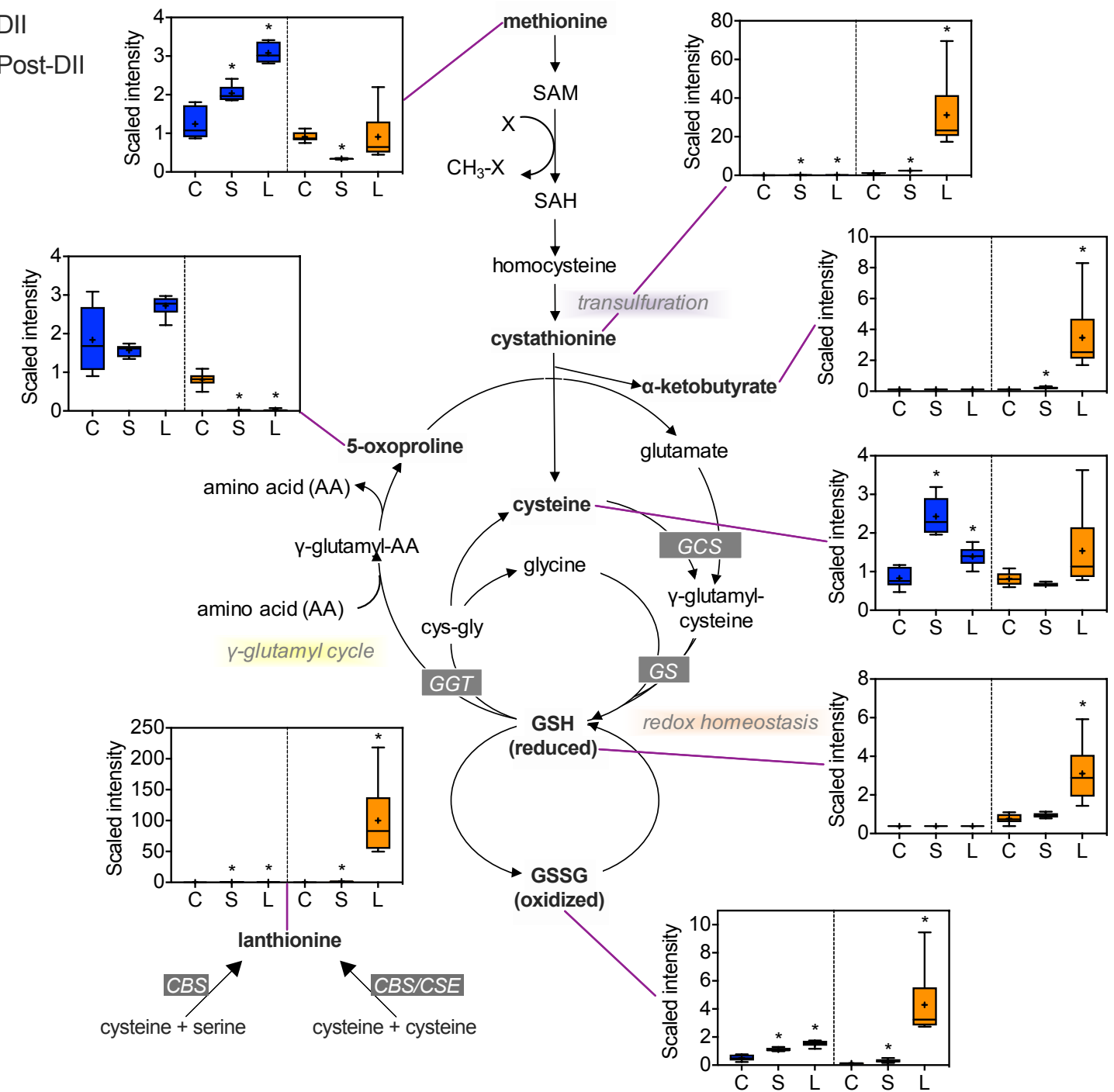

Figure 4.5. Indications of altered transsulfuration activity and antioxidant utilization in DII and post-DIl embryos. Box plot data illustrating metabolites contributing to methionine, cysteine, and glutathione metabolism. Data (scaled intensity) are presented as box plots with a line drawn at the median (mean indicated by a plus symbol) and the box indicating upper and lower quartiles. Bars are minimums and maximums of the distribution. Vertical dotted lines separate data from DII (blue) and post-DIl embryos (orange). Data are organized by aerial exposure treatment $(C$, control; $S$, short-term; $L$, long-term). Asterisks represent treatments that were significantly different from the control group (Welch's two-sample t-test, $P<0.05$ ). Each treatment had six biological replicates $(N=6)$ containing 25 embryos each. See Figure 4.1 for more information on sampling. Raw values for each metabolite were scaled to set the median across all samples to 1 and were normalized to DNA content. Relevant enzymes are included (grey boxes). CBS, cystathionine $\beta$-synthase; CSE, cystathionine $\gamma$-lyase; GCS, gamma-glutamylcysteine synthetase; GS, glutathione synthase; GGT, gamma-glutamyl transferase. 

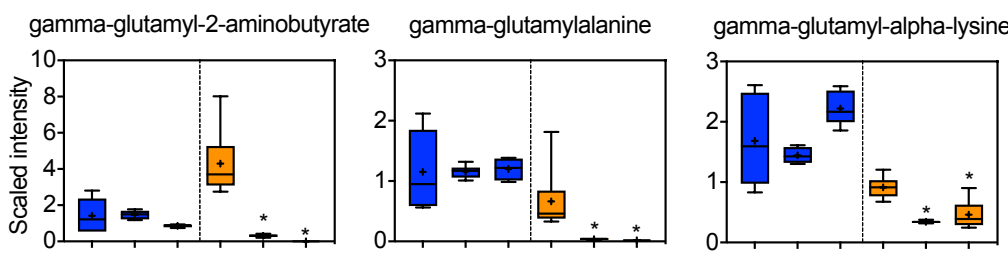

gamma-glutamylglutamate

gamma-glutamylglutamine

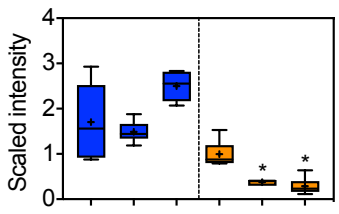

gamma-glutamylglycine

gamma-glutamylhistidine

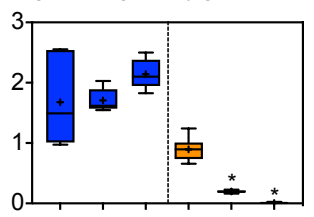

DII


gamma-glutamylisoleucine*

Post-DII

gamma-glutamylleucine



gamma-glutamylmethionine
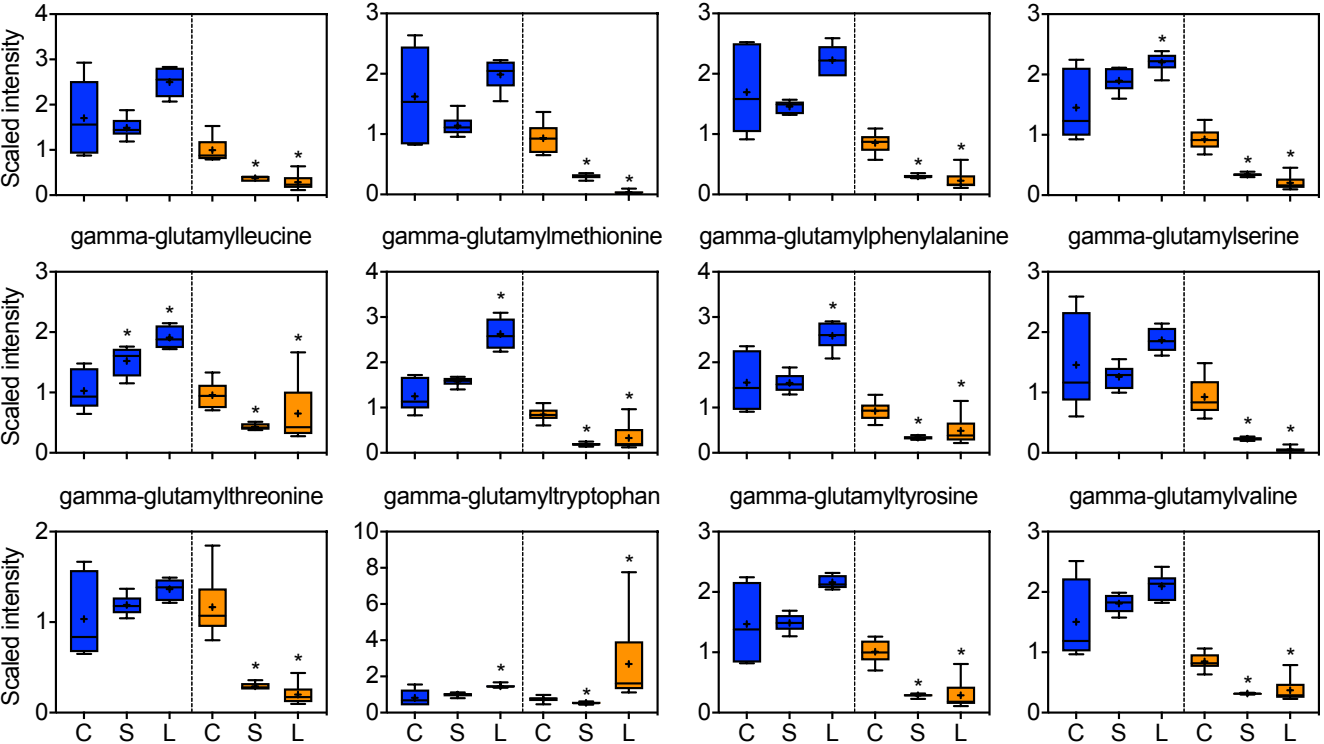

gamma-glutamyltryptophan

gamma-glutamyltyrosine
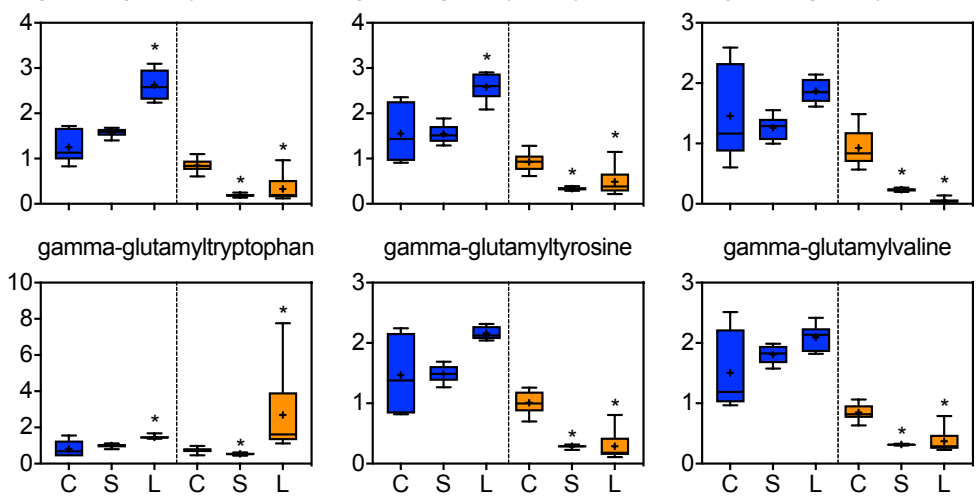

gamma-glutamylvaline

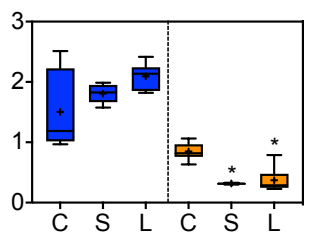

Figure 4.6. Changes in gamma-glutamyl amino acid metabolism in DII and post-DII embryos. Box plots illustrating metabolites involved in gamma-glutamyl amino acid metabolism that were significantly altered in at least one stage. Data (scaled intensity) are presented as box plots with a line drawn at the median (mean indicated by a plus symbol) and the box indicating upper and lower quartiles. Bars are minimums and maximums of the distribution. Vertical dotted lines separate data from DII (blue) and post-DII embryos (orange). Data are organized by aerial incubation treatment ( $\mathrm{C}$, control; S, short-term; L, long-term). Asterisks represent treatments that were significantly different from the control group (Welch's two-sample t-test, $P<0.05$ ). Each treatment had six biological replicates $(N=6)$ containing 25 embryos each. See Figure 4.1 for more information on sampling. Raw values for each metabolite were scaled to set the median across all samples to 1 and were normalized to DNA content. Gamma-glutamylisoleucine (asterisk) had not been confirmed based on a standard, but Metabolon is confident in the identity. 
DII

Post-DII
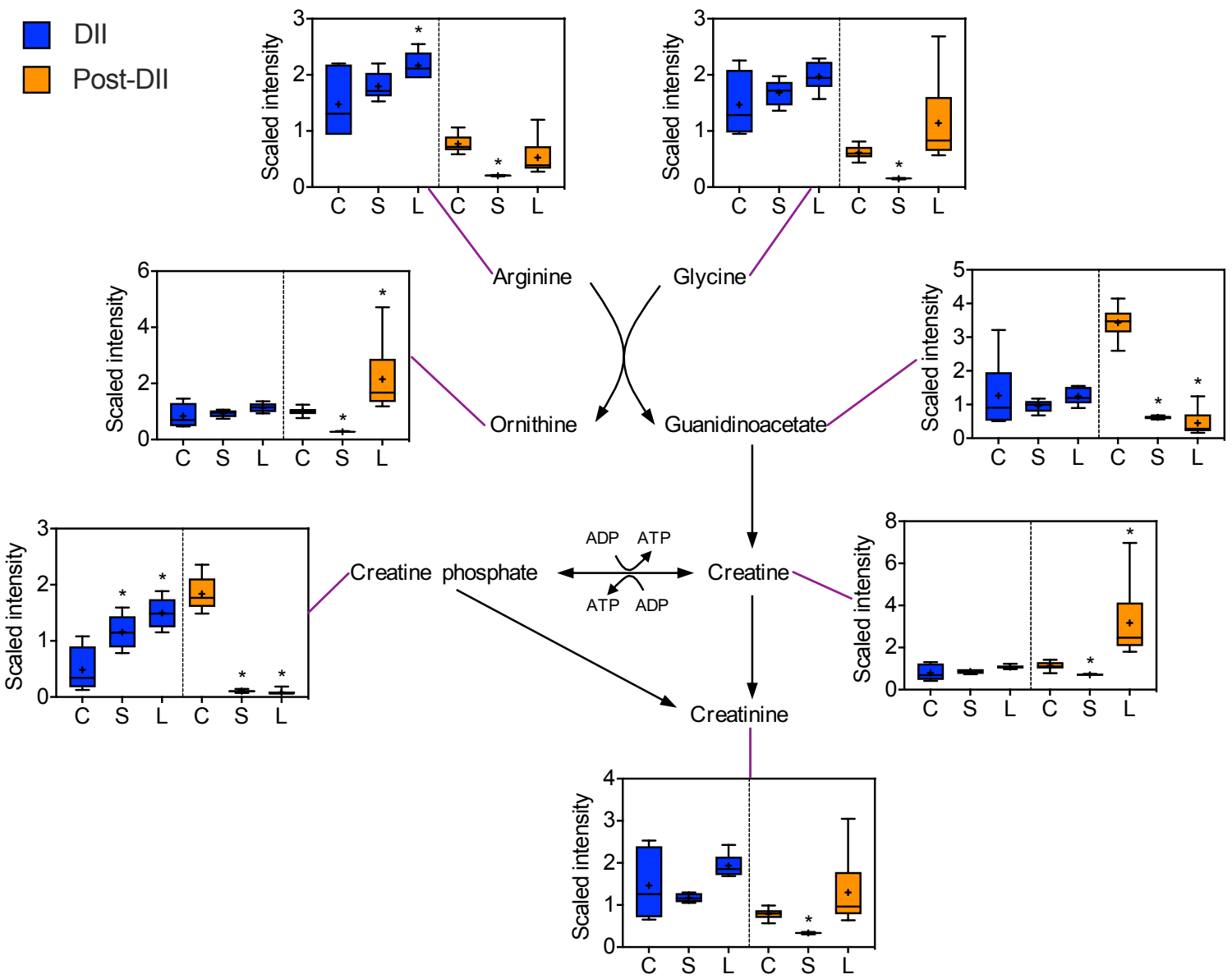

Figure 4.7. Changes in creatine metabolism in DII and post-DII embryos. Box plots illustrating metabolites involved in creatine metabolism. Data (scaled intensity) are presented as box plots with a line drawn at the median (mean indicated by a plus symbol) and the box indicating upper and lower quartiles. Bars are minimums and maximums of the distribution. Vertical dotted lines separate data from DII (blue) and post-DII embryos (orange). Data are organized by aerial incubation treatment (C, control; S, short-term; L, long-term). Asterisks represent treatments that were significantly different from the control group (Welch's two-sample t-test, $P<0.05)$. Each treatment had six biological replicates $(N=6)$ containing 25 embryos each. See Figure 4.1 for more information on sampling. Raw values for each metabolite were scaled to set the median across all samples to 1 and were normalized to DNA content. 


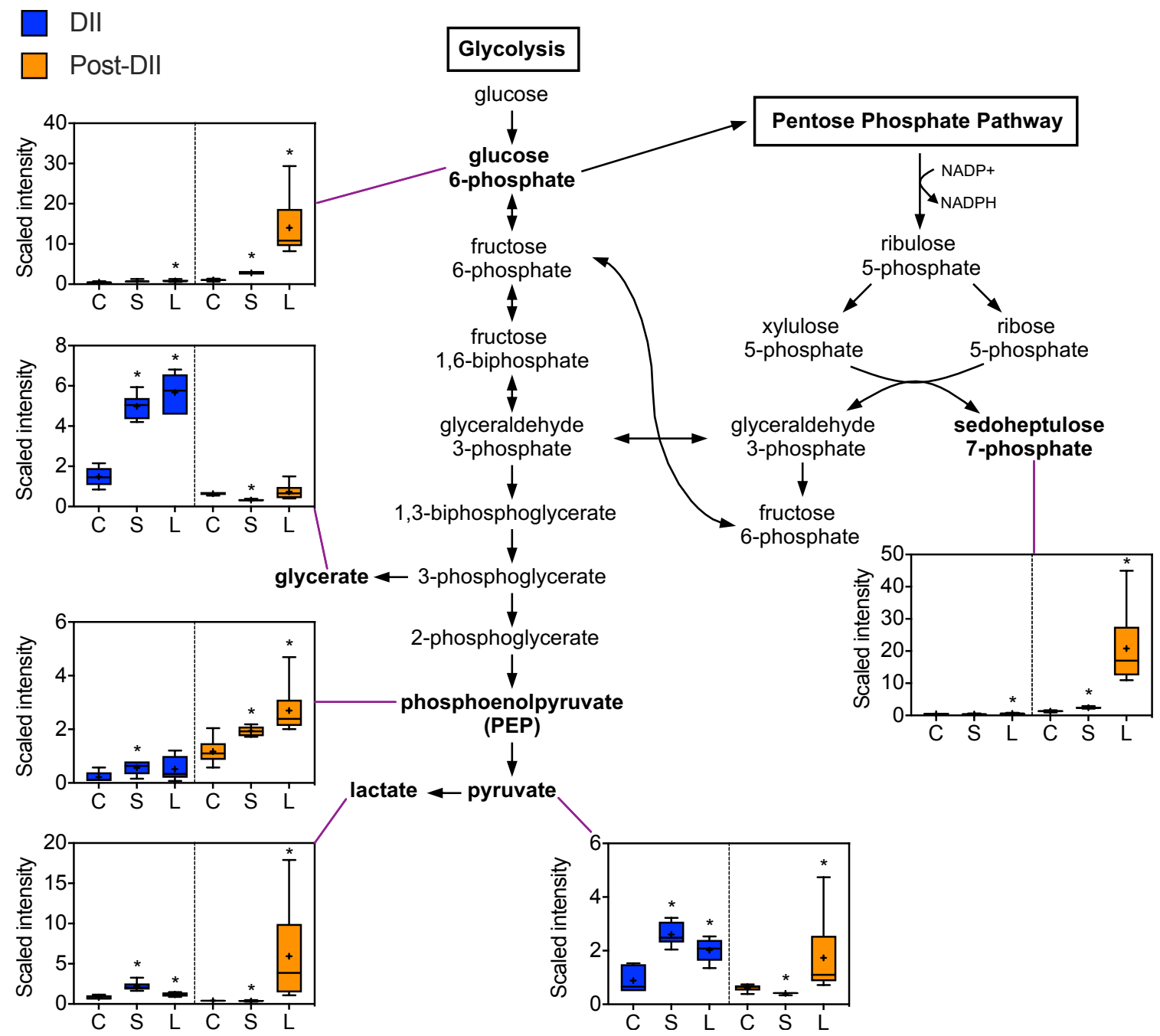

Figure 4.8. Changes in glycolysis and pentose phosphate pathway metabolism in DII and post-DIl embryos. Box plots illustrating metabolites involved in glycolysis and pentose phosphate pathway (PPP) metabolism. Data (scaled intensity) are presented as box plots with a line drawn at the median (mean indicated by a plus symbol) and the box indicating upper and lower quartiles. Bars are minimums and maximums of the distribution. Vertical dotted lines separate data from DII (blue) and post-DIl embryos (orange). Data are organized by aerial incubation treatment ( $\mathrm{C}$, control; S, short-term; L, long-term). Asterisks represent treatments that were significantly different from the control group (Welch's two-sample t-test, $P<0.05$ ). Each treatment had six biological replicates $(N=6)$ containing 25 embryos each. See Figure 4.1 for more information on sampling. Raw values for each metabolite were scaled to set the median across all samples to 1 and were normalized to DNA content. 


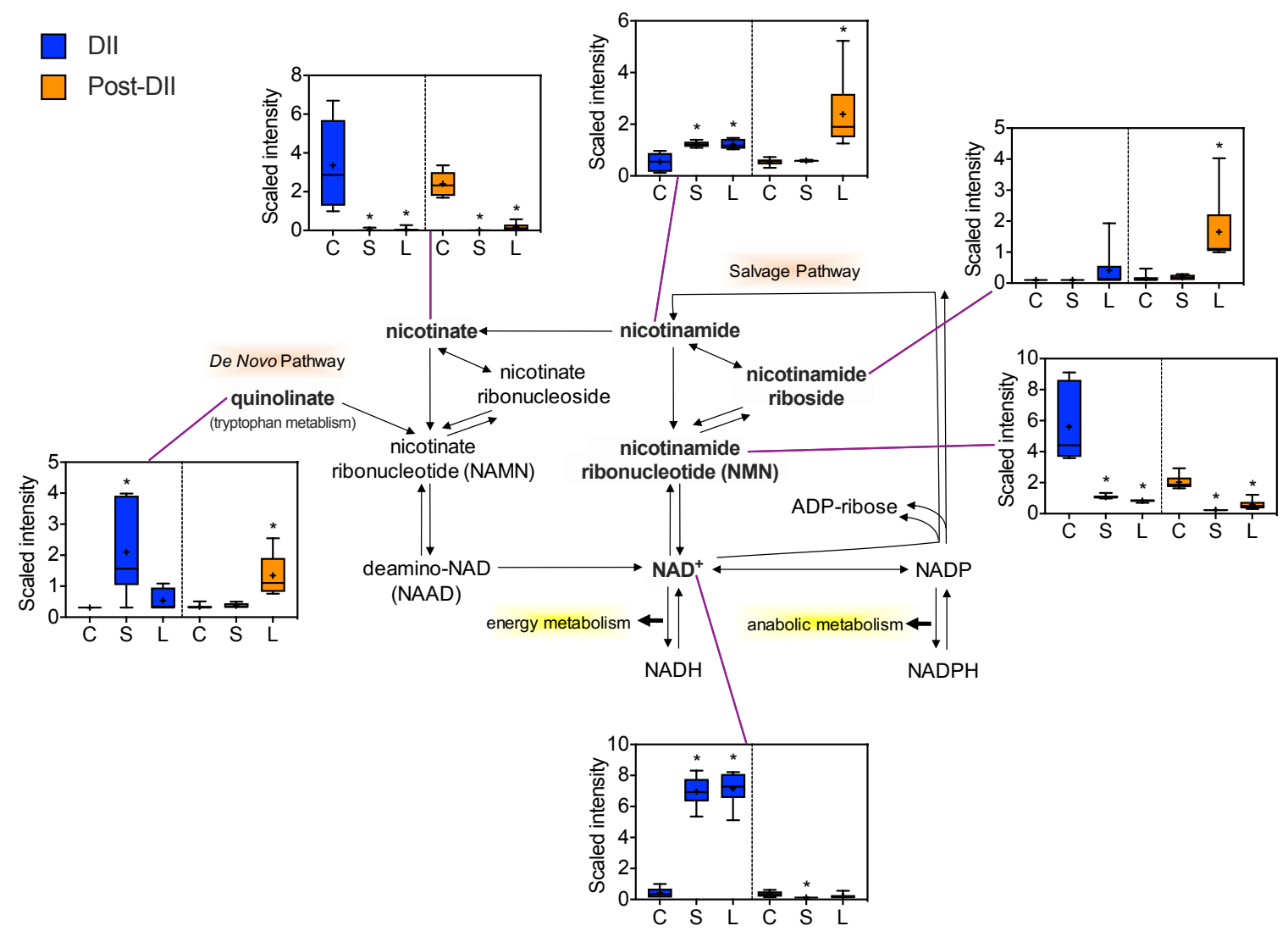

Figure 4.9. Changes in nicotinate and nicotinamide metabolism in DII and post-DII embryos. Box plots illustrating metabolites contributing to nicotinate and nicotinamide metabolism. Data (scaled intensity) are presented as box plots with a line drawn at the median (mean indicated by a plus symbol) and the box indicating upper and lower quartiles. Bars are minimums and maximums of the distribution. Vertical dotted lines separate data from DII (blue) and post-DIl embryos (orange) embryos. Data are organized by aerial incubation treatment (C, control; S, short-term; L, long-term). Asterisks represent treatments that were significantly different from the control group (Welch's two-sample t-test, $P<0.05$ ). Each treatment had six biological replicates $(N=6)$ containing 25 embryos each. See Figure 4.1 for more information on sampling. Raw values for each metabolite were scaled to set the median across all samples to 1 and were normalized to DNA content. 


\section{Chapter 5}

\section{GABA daba done: Summary and future directions}

The ability for vertebrates to survive without oxygen for prolonged periods of time is rare and astonishing. Perhaps equally as astounding and rare is survival of aquatic vertebrates without water. Remarkably, Austrofundulus limnaeus experiences both of these stresses as part of their normal embryonic development. Previous work in A. limnaeus showed accumulation of GABA in response to anoxia. The conserved nature of GABA accumulation in all groups of anoxia-tolerant vertebrates warranted the exploration into the role of GABA in $A$. limnaeus during anoxia. Exploring GABA production and its role during anoxia promised to generate novel information regarding its role in stress tolerance of $A$. limnaeus. Although work in other anoxia-tolerant systems suggested roles for GABA during anoxia, the relationship of GABA to anoxia tolerance in A. limnaeus has always been speculative until this project. Perhaps the most exciting outcome of this project was being the first study to show that inhibition of GABA metabolism directly relates to survival time in anoxia. This project also revealed many aspects of survival during dehydration stress, including the first evidence of oxygen consumption rates in A. limnaeus embryos during aerial incubation, a portion of their lifecycle in which they undoubtedly spend substantial time. The physiological and molecular responses of $A$. limnaeus embryos to aerial incubation further expanded our knowledge of the unique biology of this system. Performing a metabolomics analysis on multiple stages of embryos in response 
to dehydration stress has provided a broad assessment of the metabolites accumulated in extreme dehydration tolerance and a foundation of data for further studies.

We hypothesized that anoxia tolerance is a pre-adaptation that led to the evolution of dehydration tolerance in A. limnaeus, thus embryos would respond similarly at the molecular level to combat each individual stressor. There is undoubtedly overlap in the molecular responses to anoxia and dehydration stress, as demonstrated by GABA production during both stresses. However, the likelihood that GABA is serving all of the same functions during each stressor is unlikely. GABA is seen to accumulate in millimolar quantities during anoxia, a time where metabolism is severely depressed, whereas GABA likely (based on our knowledge of baseline levels and fold changes from metabolomics data) accumulates in micromolar levels during dehydration stress, a time where metabolism is high. This is not to say that micromolar concentrations are not biologically relevant, as these are the levels accumulated in other anoxia-tolerant organisms in response to anoxia, but there appears to be other major metabolic pathways at play that may further explain the extreme tolerance of embryos to dehydration stress. Although this project has elucidated various roles for GABA and uncovered other potential metabolic pathways aiding in survival during aerial incubation, there are many future studies that can be drawn from the data presented here. 


\section{GABA and lactate - multifunctional stress metabolites?}

This is the first extensive study of long-term anoxia and aerobic recovery in A. limnaeus embryos. We investigated multiple embryological stages across development that range in their tolerance of anoxia. All developmental stages investigated responded to anoxia by accumulating significant amounts of lactate, but only those stages with greater anoxia-tolerances (DII, WS 36, and WS 40) accumulated GABA. The most anoxia-sensitive stage investigated (WS 42) failed to accumulate GABA and also failed to recover from anoxia. Our findings suggest that embryos seemingly go unharmed and without developmental defect following long-term anoxia. However, recovery from anoxia appears to be slow with lactate and GABA levels not returning to pre-anoxia levels until after at least a week of aerobic recovery. This slow reduction of both lactate and GABA may be an adaptive and protective mechanism for supporting survival of long-term anoxia.

We hypothesized that anoxia-tolerant stages would have higher basal enzymatic activity responsible for GABA production than anoxia-sensitive stages,

thus being able to survive longer and produce more GABA in response to stress. However, we saw an inverse relationship between normoxic GAD activity and anoxia tolerance; embryos with the highest GAD activity (WS 42) produced negligible GABA in anoxia. Although GABA metabolism appears to be critical for long-term survival in anoxia, the innate capacity for high GAD activity does not correspond to anoxia tolerance. Through pharmacological inhibition experiments of enzymes responsible for the production (GAD) and degradation of GABA 
(GABA-transaminase and succinic semialdehyde dehydrogenase), we showed for the first time that GABA metabolism affects anoxia tolerance in $A$. limnaeus embryos. Inhibition of enzymes responsible for both production and degradation of GABA led to decreased survival in the most anoxia-tolerant stage (WS 36). Interestingly, the effects of decreased GABA production on survival are not obvious until a few weeks of anoxia, suggesting a role in long-term rather than short-term survival. Gene expression patterns of an abundance of GABA and lactate transporters in A. limnaeus embryos support the capability for intraembryonic or intercellular shuttling of lactate and GABA. Together, our results support a role for GABA as an intermediate product and not a metabolic end product. We proposed multiple roles for GABA during anoxia and aerobic recovery in A. limnaeus embryos: serving as a neurotransmitter, an energy source, and an antioxidant. Recent advancements in non-metabolic functions of GABA and lactate have shown they both can act as inhibitors of histone deacetylase (HDAC), and that lactate can serve to increase transcription through lactylation of histones (Xing et al., 2019; Zhang et al., 2019). GABA and lactate both gradually decrease during aerobic recovery, which could be a coordinated effort to help return gene expression patterns back to normal.

Although GABA and lactate may not be accumulating at levels seen in anoxia, they could be serving several important and similar functions during dehydration. Even at the micromolar range, GABA and lactate can function as important neuroprotective agents. The accumulation of other neuroprotective compounds during dehydration further enforces the need for $A$. limnaeus 
embryos to protect themselves from neural injury. As previously mentioned, both of these metabolites can function as epigenetic modifiers. Epigenetic changes through methylation and histone modifications can help cells control expression of genes by precisely turning them on or off, which could help explain the abundant changes in metabolite profiles we see in response to dehydration stress. Embryos also accumulate 2-hydroxyglutarate, a known epigenetic modifier, which further suggests a role for metabolite-driven control of gene expression in survival. Deducing the specific functions of GABA and lactate are playing in response to dehydration requires further studies, but it is clear there are several promising functions which could aid in their survival.

\section{Terrestrial eggs in an aquatic vertebrate?}

Despite knowledge of the unprecedented ability of annual killifish embryos to survive long periods of aerial exposure, this is the first time that dehydration tolerance has been measured under controlled conditions across development. We show the remarkable ability for DII embryos to survive over a year and a half without water, exhibiting a lethal time to $50 \%$ mortality ( $\left.L T_{50}\right)$ of $325 \mathrm{~d}$. Despite having similar anoxia tolerance to DII embryos, WS 36 embryos only survive a few months without water, exhibiting an $\mathrm{LT}_{50}$ of $84 \mathrm{~d}$. Even the most sensitive stages to dehydration stress (WS 40 and WS 42) were able to survive $100 \mathrm{~d}$ without water, exhibiting an $\mathrm{LT}_{50}$ of 28 and $29 \mathrm{~d}$, respectively. Perhaps most remarkable is the ability for embryos of this aquatic vertebrate to survive in terrestrial environments for as long as that of amniotic terrestrial eggs. In 
addition, we measured oxygen consumption rates of embryos incubated in aqueous and aerial conditions. Embryos in DII respond to dehydration stress by increasing rates of oxygen consumption. On the other hand, post-diapause II embryos either exhibit the same or reduced rates of oxygen consumption when compared to aqueous embryos. Aerial incubation coupled with hypoxia causes some embryos to arrest development. The stage-specific response of annual killifish embryos to dehydration stress are consistent with an intrinsic bet-hedging strategy in embryos that would increase developmental variation in a potentially adaptive manner. The extreme tolerance of DII embryos to dehydration stress observed suggest it is likely the stage responsible for survival of $A$. limnaeus populations during the unpredictable dry seasons.

A major focus of this thesis was to gain insight into the mechanisms facilitating survival during aerial exposure by examining the metabolite profiles of dormant and actively developing embryos. There is strong evidence for differences in metabolic profiles in the study based on developmental stage and length of aerial exposure. Actively developing embryos exhibit more robust change, however, dormant embryos respond in an active manner by significantly altering their metabolic profile. We identify a number of metabolites that accumulate in both embryos that may be playing an important role in survival, including the identification of known antioxidants and neuroprotectants. In addition, we identify unique metabolites not yet discussed in the dehydration literature, such as the non-proteinogenic amino acid lanthionine and oncometabolite 2-hydroxyglutarate. Despite high oxygen availability, embryos 
accumulate the anaerobic glycolytic end-product lactate and neurotransmitter GABA. These metabolites may be acting as extracellular signaling molecules through the activation of G-protein-coupled receptors (GPCRs) (Husted et al., 2017). Together, this paper offers an overview of the metabolic changes occurring that may support embryonic survival during dehydrating conditions, which can be functionally tested using genetic and pharmacological approaches.

Embryos of $A$. limnaeus likely spend a majority of their life span aerially incubated and thus must deal with waste products in a unique way. During aerial incubation, embryos are unable to shuttle waste to the outside environment or even into the perivitelline space, as all fluid is void. Instead, embryos most likely rely on mechanisms very similar to those of amniotic eggs, which is to transport waste away from the developing embryo into extraembryonic compartments, such as the yolk sac or the extraembryonic fluid. In essence, the yolk sac and extraembryonic fluid serve analogous roles with the allantois and amniotic fluid of amniotes. The extraordinary ability for embryos to survive terrestrial conditions paired with potential waste management mechanisms analogous to amniotic eggs suggest $A$. limnaeus may represent an intermediate in the evolution of amniotes. This is further bolstered by $A$. limnaeus embryos accumulating many compounds that are usually found in the amniotic and allantoic fluid in terrestrial species. Further studies into the actions of these compartments during aerial incubation might lead to interesting discoveries on the waste regulation and evolution of this complex system. 


\section{Future research directions}

One of the most interesting results from this thesis was the observation of decreased survival of embryos in anoxia when production and degradation of GABA was inhibited. This observation was shown in the anoxia-tolerant and metabolically active WS 36 embryos. It would be interesting to see if similar results are observed in dormant DII embryos that exhibit a similar anoxia tolerance. We showed that GABA appears to be aiding in long-term survival, so it would be interesting to see if inhibition of GABA production or degradation would reduce the already short anoxia tolerance of WS 40 embryos $\left(L T_{50}=7 \mathrm{~d}\right)$. Supplementation of GABA into the embryo medium to potentially extend the tolerance of anoxia-sensitive embryos may seem like a logical next step.

However, the enveloping cell layer of $A$. limnaeus embryos appears to be nearly impermeable to amino acids even when using a vehicular aid such as DMSO. Thus, it is unlikely that GABA would permeate in sufficient amounts. A possible next step is to test this hypothesis using embryo-derived anoxia-tolerant cell lines. There are currently two cell lines of neural origin derived from embryos of A. limnaeus (PSU-AL-WS4ONE and WS36-2) that can survive for more than 49 days without oxygen. A cell model would allow us to easily treat cells with GABA to determine if GABA supplementation extends anoxia tolerance. This would be especially interesting in these cell lines as they have not been seen to accumulate GABA despite surviving for so long in anoxia. Further, we can compare the effects of GABA on anoxia-sensitive cell lines derived from mouse, monkey, or human to see if we can extend their tolerances. 
A difficulty with measuring metabolites in whole embryos is accounting for the many compartments in which these metabolites can exist in (e.g., perivitelline fluid, embryonic tissue, yolk sac, and the extraembryonic cavity bounded by the enveloping cell layer). We suggest the possibility that GABA and lactate are being shuttled from the embryonic tissue to the yolk sac, thus localization of these metabolites is an important next step. Further studies into GABA and lactate presence in yolk and cellular fractions of embryos will help elucidate the role the yolk may be playing as a buffering or storage system. We observed many gene transcripts associated with GABA and lactate transporters, as well as GABA receptors, though we do not know their function or localization during anoxia or normal development. Immunohistochemistry would allow for localization of these proteins as well as localization of GABA and lactate themselves, which would further illuminate our understanding of anoxia survival. We also suggested the slow decrease in GABA and lactate during aerobic recovery from anoxia may be mechanisms for mitigating reactive oxygen species (ROS) production, thus investigating ROS levels in embryos as it relates to GABA and lactate levels during aerobic recovery will also be an important next step.

The work in this thesis revealed differences in the rate of post-DII development in embryos during dehydration exposure. When oxygen was limited, but still above the measured $\mathrm{P}_{\text {crit }}$ of $A$. limnaeus embryos, aerial incubation led to developmental arrest in a proportion of embryos. However, post-DIl embryos developing with ample oxygen (in large desiccators) develop 
synchronously. Beyond the evidence in this dissertation, little is known about development during aerial exposure and what controls whether an embryo will arrest development. Anecdotal evidence shows embryos that break DII while on filter pads, though uncommon, develop drastically slower than embryos that are already post-DII when placed on filter pads. Although it appears as though embryos develop unharmed during aerial incubation, further research needs to be done to examine embryos and their morphology as they develop in aerial conditions.

During this dissertation work, embryos were never rehydrated following long-term dehydration, thus we do not know how embryos will react following reintroduction of water. From a developmental and ecological perspective, it would be interesting to see if reintroduction of water to DII embryos causes them to break DII and continue developing. This would mimic what we assume they experience in the wild and thus a normal part of their life cycle. As we know there are a lot of dramatic changes in cell physiology occurring during transition from aqueous to aerial conditions, there undoubtedly are changes occurring when going from a dehydrated state back to hydrated. Going forward, it could be of interest to examine how the metabolome of dehydrated embryos responds upon rehydration. Now that we have a foundation of metabolites to compare to, we can perform targeted assays to measure the abundance of certain metabolites during aerial incubation and rehydration that we believe may be playing key roles in survival. 
Continued research on non-model organisms holds promise for better understanding how organisms have adapted to overcome physiological challenges imposed by environmental stress. Since many of the mechanisms utilized by $A$. limnaeus embryos in response to anoxia and dehydration stress are conserved across taxa, it is likely that these same mechanisms can be exploited in sensitive models, such as humans, which could lead to promising medical applications. 


\section{References}

Aktuğ, T., Uçan, B., Olguner, M., Akgür, F., Özer, E., Calişkan, S. and Önvural, B. (1998). Amnio-allantoic fluid exchange for the prevention of intestinal damage in gastroschisis III: determination of the waste products removed by exchange. European journal of pediatric surgery 8, 326-328.

Bachhawat, A. K. and Yadav, S. (2018). The glutathione cycle: Glutathione metabolism beyond the $\gamma$-glutamyl cycle. IUBMB Life 70, 585-592.

Bayley, M. and Holmstrup, M. (1999). Water vapor absorption in arthropods by accumulation of myoinositol and glucose. Science 285, 19091911.

Bayley, M., Petersen, S. O., Knigge, T., Köhler, H.-R. and Holmstrup, M. (2001). Drought acclimation confers cold tolerance in the soil collembolan Folsomia candida. Journal of Insect Physiology 47, 1197-1204.

Ben-Ari, Y. (2002). Excitatory actions of GABA during development: the nature of the nurture. Nature Reviews Neuroscience 3, 728-740.

Ben-Menachem, E. (2011). Mechanism of action of vigabatrin: correcting misperceptions. Acta neurologica scandinavica 124, 5-15.

Berthet, C., Lei, H., Thevenet, J., Gruetter, R., Magistretti, P. J. and Hirt, L. (2009). Neuroprotective role of lactate after cerebral ischemia. Journal of Cerebral Blood Flow \& Metabolism 29, 1780-1789.

Blackstone, E., Morrison, M. and Roth, M. B. (2005). $\mathrm{H}_{2} \mathrm{~S}$ induces a suspended animation-like state in mice. Science 308, 518.

Bosco, M. C., Rapisarda, A., Massazza, S., Melillo, G., Young, H. and Varesio, L. (2000). The tryptophan catabolite picolinic acid selectively induces the chemokines macrophage inflammatory protein-1 $\alpha$ and-1 $\beta$ in macrophages. The Journal of Immunology 164, 3283-3291.

Boutilier, R. G. (2001). Mechanisms of cell survival in hypoxia and hypothermia. Journal of Experimental Biology 204, 3171-3181.

Braidy, N., Guillemin, G. J., Mansour, H., Chan-Ling, T., Poljak, A. and Grant, R. (2011). Age related changes in NAD+ metabolism oxidative stress and Sirt1 activity in wistar rats. PLOS ONE 6. 
Bundgaard, A., Ruhr, I. M., Fago, A. and Galli, G. L. (2020). Metabolic adaptations to anoxia and reoxygenation: new lessons from freshwater turtles and Crucian carp. Current Opinion in Endocrine and Metabolic Research.

Buttgereit, F. and Brand, M. D. (1995). A hierarchy of ATP-consuming processes in mammalian cells. Biochemical Journal 312, 163-167.

Canto, C., Menzies, K. J. and Auwerx, J. (2015). NAD+ metabolism and the control of energy homeostasis: a balancing act between mitochondria and the nucleus. Cell Metabolism 22, 31-53.

Cao, J., Barbosa, J. M., Singh, N. K. and Locy, R. D. (2013). GABA shunt mediates thermotolerance in Saccharomyces cerevisiae by reducing reactive oxygen production. Yeast 30, 129-144.

Cerutti, R., Pirinen, E., Lamperti, C., Marchet, S., Sauve, A. A., Li, W., Leoni, V., Schon, E. A., Dantzer, F. and Auwerx, J. (2014). NAD+-dependent activation of Sirt1 corrects the phenotype in a mouse model of mitochondrial disease. Cell Metabolism 19, 1042-1049.

Chapeville, F. and Fromageot, P. (1967). "Vestigial" enzymes during embryonic development. Advances in Enzyme Regulation 5, 155-158.

Chapman, G. A., Denton, D. L. and Lazorchak, J. M. (1995). Short-Term Methods for Estimating the Chronic Toxicity of Effluents and Receiving Waters to West Coast Marine and Estuarine Organisms, vol. 1 (ed. N. E. R. L.-. Cincinnati), pp. 1-673. Cincinnati, OH 45268.

Charitou, P., Rodriguez-Colman, M., Gerrits, J., van Triest, M., Koerkamp, M. G., Hornsveld, M., Holstege, F., Verhoeven-Duif, N. M. and Burgering, B. M. (2015). FOXOs support the metabolic requirements of normal and tumor cells by promoting IDH1 expression. EMBO Reports 16, 456-466.

Chennault, T. and Podrabsky, J. E. (2010). Aerobic and anaerobic capacities differ in embryos of the annual killifish Austrofundulus limnaeus that develop on alternate developmental trajectories. Journal of Experimental Zoology Part A: Ecological Genetics and Physiology 313A, 587-596.

Chiku, T., Padovani, D., Zhu, W., Singh, S., Vitvitsky, V. and Banerjee, R. (2009). H2S biogenesis by human cystathionine $y$-lyase leads to the novel sulfur metabolites lanthionine and homolanthionine and is responsive to the grade of hyperhomocysteinemia. Journal of Biological Chemistry 284, 11601-11612. 
Chung, H. and Carroll, S. B. (2015). Wax, sex and the origin of species: dual roles of insect cuticular hydrocarbons in adaptation and mating. BioEssays $37,822-830$.

Coleman, S. T., Fang, T. K., Rovinsky, S. A., Turano, F. J. and MoyeRowley, W. S. (2001). Expression of a glutamate decarboxylase homologue is required for normal oxidative stress tolerance in Saccharomyces cerevisiae. Journal of Biological Chemistry 276, 244-250.

Crowe, J. H., Crowe, L. M. and Chapman, D. (1984). Preservation of membranes in anhydrobiotic organisms: the role of trehalose. Science 223, 701703.

Crowe, J. H. and Madin, K. A. (1974). Anhydrobiosis in tardigrades and nematodes. Transactions of the American Microscopical Society 93, 513-524.

Danks, H. V. (2000). Dehydration in dormant insects. Journal of Insect Physiology 46, 837-852.

DiMichele, L. and Powers, D. A. (1984). The relationship between oxygen consumption rate and hatching in Fundulus heteroclitus. Physiological Zoology 57, 46-51.

Elrod, J. W., Calvert, J. W., Morrison, J., Doeller, J. E., Kraus, D. W., Tao, L., Jiao, X., Scalia, R., Kiss, L. and Szabo, C. (2007). Hydrogen sulfide attenuates myocardial ischemia-reperfusion injury by preservation of mitochondrial function. Proceedings of the National Academy of Sciences of the United States of America 104, 15560-15565.

Erkut, C. and Kurzchalia, T. V. (2015). The C. elegans dauer larva as a paradigm to study metabolic suppression and desiccation tolerance. Planta 242, 389-396.

Erkut, C., Penkov, S., Khesbak, H., Vorkel, D., Verbavatz, J.-M., Fahmy, K. and Kurzchalia, T. V. (2011). Trehalose renders the dauer larva of Caenorhabditis elegans resistant to extreme desiccation. Current Biology 21, 1331-1336.

Ernst, V., Levin, D. H. and London, I. M. (1978). Inhibition of protein synthesis initiation by oxidized glutathione: activation of a protein kinase that phosphorylates the a subunit of eukaryotic initiation factor 2. Proceedings of the National Academy of Sciences of the United States of America 75, 4110-4114. 
Fernandez-Pol, J., Bono, V. H. and Johnson, G. S. (1977). Control of growth by picolinic acid: differential response of normal and transformed cells. Proceedings of the National Academy of Sciences of the United States of America 74, 2889-2893.

Fishman, A. P., Galante, R. J., Winokur, A. and Pack, A. I. (1992). Estivation in the African Lungfish. Proceedings of the American Philosophical Society 136, 61-72.

Fontana, M., Ricci, G., Solinas, S., Antonucci, A., Serao, I., Dupre, S. and Cavallini, D. (1990). $\left[{ }^{35} S\right]$ Lanthionine ketimine binding to bovine brain membranes. Biochemical and Biophysical Research Communications 171, 480486.

França, M., Panek, A. and Eleutherio, E. (2007). Oxidative stress and its effects during dehydration. Comparative Biochemistry and Physiology Part A: Molecular \& Integrative Physiology 146, 621-631.

Fraser, E. J., Bosma, P. T., Trudeau, V. L. and Docherty, K. (2002). The Effect of Water Temperature on the GABAergic and Reproductive Systems in Female and Male Goldfish (Carassius auratus). General and Comparative Endocrinology 125, 163-175.

Fu, X., Chin, R. M., Vergnes, L., Hwang, H., Deng, G., Xing, Y., Pai, M. Y., Li, S., Ta, L. and Fazlollahi, F. (2015). 2-Hydroxyglutarate inhibits ATP synthase and mTOR signaling. Cell Metabolism 22, 508-515.

Fuery, C. J., Withers, P. C. and Guppy, M. (1998). Protein synthesis in the liver of Bufo marinus: Cost and contribution to oxygen consumption. Comparative Biochemistry and Physiology 119A, 459-467.

Furness, A. I., Lee, K. and Reznick, D. N. (2015). Adaptation in a variable environment: Phenotypic plasticity and bet-hedging during egg diapause and hatching in an annual killifish. Evolution 69, 1461-1475.

Gilby, K., Sydserff, S. and Robertson, H. (2005). Differential neuroprotective effects for three GABA-potentiating compounds in a model of hypoxia-ischemia. Brain Research 1035, 196-205.

Gleeson, T. T. (1985). Glycogen synthesis from lactate in skeletal muscle of the lizard Dipsosaurus dorsalis. Journal of Comparative Physiology 156, 277284. 
Goldlust, A., Su, T.-Z., Welty, D., Taylor, C. and Oxender, D. (1995). Effects of anticonvulsant drug gabapentin on the enzymes in metabolic pathways of glutamate and GABA. Epilepsy Research 22, 1-11.

Goldstein, B. and King, N. (2016). The Future of Cell Biology: Emerging Model Organisms. Trends in Cell Biology 26, 818-824.

Gomes, A. P., Price, N. L., Ling, A. J., Moslehi, J. J., Montgomery, M. K., Rajman, L., White, J. P., Teodoro, J. S., Wrann, C. D. and Hubbard, B. P. (2013). Declining NAD+ induces a pseudohypoxic state disrupting nuclear-mitochondrial communication during aging. Cell 155, 1624-1638.

Grant, R., Coggan, S. and Smythe, G. (2009). The physiological action of picolinic acid in the human brain. International Journal of Tryptophan Research 2, IJTR-S2469.

Hand, S. C. (1998). Quiescence in Artemia franciscana embryos: reversible arrest of metabolism and gene expression at low oxygen levels. Journal of Experimental Biology 201, 1233-1242.

Hand, S. C. and Podrabsky, J. E. (2000). Bioenergetics of diapause and quiescence in aquatic animals. Thermochimica Acta 349, 31-42.

Hansen, J. M., Go, Y.-M. and Jones, D. P. (2006). Nuclear and mitochondrial compartmentation of oxidative stress and redox signaling. Annual Review of Pharmacology and Toxicology 46, 215-234.

Hensley, K., Venkova, K. and Christov, A. (2010). Emerging biological importance of central nervous system lanthionines. Molecules 15, 5581-5594.

Husted, A. S., Trauelsen, M., Rudenko, O., Hjorth, S. A. and Schwartz, T. W. (2017). GPCR-mediated signaling of metabolites. Cell Metabolism 25, 777796.

Hylemon, P. B., Zhou, H., Pandak, W. M., Ren, S., Gil, G. and Dent, P. (2009). Bile acids as regulatory molecules. Journal of Lipid Research 50, 15091520.

Hylland, P. and Nilsson, G. E. (1999). Extracellular levels of amino acid neurotransmitters during anoxia and forced energy deficiency in crucian carp brain. Brain Research 823, 49-58. 
Inoue, M. (2016). Glutathionists in the battlefield of gamma-glutamyl cycle. Archives of Biochemistry and Biophysics 595, 61-63.

Intlekofer, A. M., Dematteo, R. G., Venneti, S., Finley, L. W., Lu, C., Judkins, A. R., Rustenburg, A. S., Grinaway, P. B., Chodera, J. D. and Cross, J. R. (2015). Hypoxia induces production of L-2-hydroxyglutarate. Cell Metabolism 22, 304-311.

Intlekofer, A. M., Wang, B., Liu, H., Shah, H., Carmona-Fontaine, C., Rustenburg, A. S., Salah, S., Gunner, M. R., Chodera, J. D. and Cross, J. R. (2017). L-2-Hydroxyglutarate production arises from noncanonical enzyme function at acidic pH. Nature Chemical Biology 13, 494.

Ippolito, J. E. and Piwnica-Worms, D. (2014). A fluorescence-coupled assay for gamma aminobutyric acid (GABA) reveals metabolic stress-induced modulation of GABA content in neuroendocrine cancer. PLoS ONE 9, e88667.

Jackson, D. (1997). Lactate accumulation in the shell of the turtle Chrysemys picta bellii during anoxia at $3^{\circ} \mathrm{C}$ and $10^{\circ} \mathrm{C}$. Journal of Experimental Biology 200, 2295-2300.

Jackson, D. C., Crocker, C. E. and Ultsch, G. R. (2000). Bone and shell contribution to lactic acid buffering of submerged turtles Chrysemys picta bellii at $3^{\circ} \mathrm{C}$. American Journal of Physiology - Regulatory, Integrative and Comparative Physiology 278, R1564-R1571.

Jackson, D. C. and Heisler, N. (1982). Plasma ion balance of submerged anoxic turtles at $3^{\circ} \mathrm{C}$ : the role of calcium lactate formation. Respiration Physiology $49,159-174$.

Jacobson, M. D. and Raff, M. C. (1995). Programmed cell death and Bcl-2 protection in very low oxygen. Nature 374, 814-816.

Johns, L., Sinclair, A. J. and Davies, J. A. (2000). Hypoxia/hypoglycemiainduced amino acid release is decreased in vitro by preconditioning. Biochemical and Biophysical Research Communications 276, 134-136.

Jorgenson, N.-C. and Schmalbruch, H. (1984). The eggs of the freshwater fish Epiplatys dageti have tight plasma membranes without intramembranous particles. Cell and Tissue Research 235, 643-646. 
Jourdain, P., Allaman, I., Rothenfusser, K., Fiumelli, H., Marquet, P. and Magistretti, P. J. (2016). L-Lactate protects neurons against excitotoxicity: implication of an ATP-mediated signaling cascade. Scientific Reports 6, 1-13.

Khan, N. A., Auranen, M., Paetau, I., Pirinen, E., Euro, L., Forsström, S., Pasila, L., Velagapudi, V., Carroll, C. J. and Auwerx, J. (2014). Effective treatment of mitochondrial myopathy by nicotinamide riboside, a vitamin B3. EMBO molecular medicine 6, 721-731.

Koppaka, V., Thompson, D. C., Chen, Y., Ellermann, M., Nicolaou, K. C., Juvonen, R. O., Petersen, D., Deitrich, R. A., Hurley, T. D. and Vasiliou, V. (2012). Aldehyde dehydrogenase inhibitors: a comprehensive review of the pharmacology, mechanism of action, substrate specificity, and clinical application. Pharmacological reviews 64, 520-539.

Kubasiak, L. A., Hernandez, O. M., Bishopric, N. H. and Webster, K. A. (2002). Hypoxia and acidosis activate cardiac myocyte death through the Bcl-2 family protein BNIP3. Proceedings of the National Academy of Sciences of the United States of America 99, 12825-12830.

Landoni, G., Zangrillo, A., Lomivorotov, V. V., Likhvantsev, V., Ma, J., De Simone, F. and Fominskiy, E. (2016). Cardiac protection with phosphocreatine: a meta-analysis. Interactive cardiovascular and thoracic surgery 23, 637-646.

Larson, J., Drew, K. L., Folkow, L. P., Milton, S. L. and Park, T. J. (2014). No oxygen? No problem! Intrinsic brain tolerance to hypoxia in vertebrates. Journal of Experimental Biology 217, 1024-1039.

Latham, T., Mackay, L., Sproul, D., Karim, M., Culley, J., Harrison, D. J., Hayward, L., Langridge-Smith, P., Gilbert, N. and Ramsahoye, B. H. (2012). Lactate, a product of glycolytic metabolism, inhibits histone deacetylase activity and promotes changes in gene expression. Nucleic Acids Research 40, 47944803.

Lees, H. J., Swann, J. R., Wilson, I. D., Nicholson, J. K. and Holmes, E. (2013). Hippurate: the natural history of a mammalian-microbial cometabolite. Journal of Proteome Research 12, 1527-1546.

Leeson, T. and Leeson, C. (1963). The chorio-allantois of the chick. Light and electron microscopic observations at various times of incubation. Journal of anatomy $97,585$. 
Li, S., Gao, D. and Jiang, Y. (2019). Function, detection and alteration of acylcarnitine metabolism in hepatocellular carcinoma. Metabolites 9, 36.

Lopez-Martinez, G., Benoit, J. B., Rinehart, J. P., Elnitsky, M. A., Lee, R. E. and Denlinger, D. L. (2009). Dehydration, rehydration, and overhydration alter patterns of gene expression in the Antarctic midge, Belgica antarctica. Journal of Comparative Physiology B 179, 481-491.

Lutz, P. L. and Milton, S. L. (2004). Negotiating brain anoxia survival in the turtle. Journal of Experimental Biology 207, 3141-3147.

Lutz, P. L. and Nilsson, G. E. (2004). Vertebrate brains at the pilot light. Respiratory Physiology and Neurobiology 141, 285-296.

Machado, B. E. and Podrabsky, J. E. (2007). Salinity tolerance in diapausing embryos of the annual killifish Austrofundulus limnaeus is supported by exceptionally low water and ion permeability. Journal of Comparative Physiology B: Biochemical, Systemic, and Environmental Physiology 177, 809820.

Martin, A. A. and Cooper, A. K. (1972). The ecology of terrestrial anuran eggs, genus Crinia (Leptodactylidae). Copeia 1972, 163-168.

Martin, K. and Carter, A. (2013). Brave new propagules: terrestrial embryos in anamniotic eggs. Integrative and Comparative Biology 53, 233-247.

Martin, K., Van Winkle, R., Drais, J. and Lakisic, H. (2004). Beachspawning fishes, terrestrial eggs, and air breathing. Physiological and Biochemical Zoology 77, 750-759.

Martin, K. L. M. (1999). Ready and waiting: delayed hatching and extended incubation of anamniotic vertebrate terrestrial eggs. American Zoologist 39, 279-288.

Martin, K. L. M., Moravek, C. L. and Walker, A. J. (2011). Waiting for a sign: extended incubation postpones larval stage in the beach spawning California grunion Leuresthes tenuis (Ayres). Environmental Biology of Fishes 91, 63-70.

Masella, R., Di Benedetto, R., Varì, R., Filesi, C. and Giovannini, C. (2005). Novel mechanisms of natural antioxidant compounds in biological 
systems: involvement of glutathione and glutathione-related enzymes. Journal of Nutritional Biochemistry 16, 577-586.

McDowall, R. M. and Charteris, S. C. (2006). The possible adaptive advantages of terrestrial egg deposition in some fluvial diadromous galaxiid fishes (Teleostei: Galaxiidae). Fish and Fisheries 7, 153-164.

Meller, C. L., Meller, R., Simon, R. P., Culpepper, K. M. and Podrabsky, J. E. (2012). Cell cycle arrest associated with anoxia-induced quiescence, anoxic preconditioning, and embryonic diapause in embryos of the annual killifish Austrofundulus limnaeus. Journal of Comparative Physiology B 182, 909-920.

Meller, C. L. and Podrabsky, J. E. (2013). Avoidance of apoptosis in embryonic cells of the annual killifish Austrofundulus limnaeus exposed to anoxia. PLOS ONE 8, e75837.

Mellor, D. and Slater, J. (1971). Daily changes in amniotic and allantoic fluid during the last three months of pregnancy in conscious, unstressed ewes, with catheters in their foetal fluid sacs. The Journal of Physiology 217, 573-604.

Minard, K. I. and McAlister-Henn, L. (2001). Antioxidant function of cytosolic sources of NADPH in yeast. Free Radical Biology and Medicine 31, 832-843.

Moravek, C. L. and Martin, K. L. (2011). Life goes on: delayed hatching, extended incubation, and heterokairy in development of embryonic California Grunion, Leuresthes tenuis. Copeia 2011, 308-314.

Morgan, C. A., Parajuli, B., Buchman, C. D., Dria, K. and Hurley, T. D. (2015). N, N-diethylaminobenzaldehyde (DEAB) as a substrate and mechanismbased inhibitor for human ALDH isoenzymes. Chemico-biological interactions 234, 18-28.

Mouchiroud, L., Houtkooper, R. H., Moullan, N., Katsyuba, E., Ryu, D., Cantó, C., Mottis, A., Jo, Y.-S., Viswanathan, M. and Schoonjans, K. (2013). The $\mathrm{NAD+} /$ sirtuin pathway modulates longevity through activation of mitochondrial UPR and FOXO signaling. Cell 154, 430-441.

Nada, S. E., Tulsulkar, J., Raghavan, A., Hensley, K. and Shah, Z. A. (2012). A derivative of the CRMP2 binding compound lanthionine ketimine provides neuroprotection in a mouse model of cerebral ischemia. Neurochemistry International 61, 1357-1363. 
Nilsson, G. E. (1992). Evidence for a role of GABA in metabolic depression during anoxia in crucian carp (Carassius carassius). Journal of Experimental Biology 164, 243-259.

Nilsson, G. E. and Lutz, P. L. (1991). Release of inhibitory neurotransmitters in response to anoxia in turtle brain. American Journal of Physiology-Regulatory, Integrative and Comparative Physiology 261, R32-R37.

Nilsson, G. E. and Lutz, P. L. (2004). Anoxia tolerant brains. Journal of Cerebral Blood Flow \& Metabolism 24, 475-486.

Nilsson, G. E., Lutz, P. L. and Jackson, T. L. (1991). Neurotransmitters and anoxic survival of the brain: A comparison of anoxia-tolerant and anoxiaintolerant vertebrates. Physiological Zoology 64, 638-652.

Nilsson, G. E. and Renshaw, G. M. C. (2004). Hypoxic survival strategies in two fishes: extreme anoxia tolerance in the North European crucian carp and natural hypoxic preconditioning in a coral-reef shark. Journal of Experimental Biology 207, 3131-3139.

Oldham, W. M., Clish, C. B., Yang, Y. and Loscalzo, J. (2015). Hypoxiamediated increases in L-2-hydroxyglutarate coordinate the metabolic response to reductive stress. Cell Metabolism 22, 291-303.

Olson, K. R. (2018). $\mathrm{H}_{2} \mathrm{~S}$ and polysulfide metabolism: conventional and unconventional pathways. Biochemical Pharmacology 149, 77-90.

Orlowski, M. and Meister, A. (1970). The y-glutamyl cycle: a possible transport system for amino acids. Proceedings of the National Academy of Sciences of the United States of America 67, 1248-1255.

Pallister, T., Jackson, M. A., Martin, T. C., Zierer, J., Jennings, A., Mohney, R. P., MacGregor, A., Steves, C. J., Cassidy, A. and Spector, T. D. (2017). Hippurate as a metabolomic marker of gut microbiome diversity: modulation by diet and relationship to metabolic syndrome. Scientific Reports 7 , $1-9$.

Pamenter, M. E., Hogg, D. W., Ormond, J., Shin, D. S., Woodin, M. A. and Buck, L. T. (2011). Endogenous $G_{A B A}$ and $G A B A_{B}$ receptor-mediated electrical suppression is critical to neuronal anoxia tolerance. Proceedings of the National Academy of Sciences of the United States of America 108, 11274-11279. 
Patra, K. C. and Hay, N. (2014). The pentose phosphate pathway and cancer. Trends in Biochemical Sciences 39, 347-354.

Perna, A. F., Anishchenko, E., Vigorito, C., Zacchia, M., Trepiccione, F., D'Aniello, S. and Ingrosso, D. (2018). Zebrafish, a novel model system to study uremic toxins: the case for the sulfur amino acid lanthionine. International Journal of Molecular Sciences 19, 1323.

Peterson, R. H. and Martin-Robichaud, D. J. (1987). Permeability of the isolated Atlantic Salmon (Salmo salar) chorion to ions as estimated by diffusion potentials. Canadian journal of Fisheries and Aquatic Sciences 44, 1635-1639.

Podrabsky, J., Riggs, C., Romney, A., Woll, S., Wagner, J., Culpepper, K. and Cleaver, T. (2017). Embryonic development of the annual killifish Austrofundulus limnaeus: An emerging model for ecological and evolutionary developmental biology research and instruction. Developmental Dynamics 246, 779-801.

Podrabsky, J., Riggs, C. and Wagner, J. (2016). Tolerance of Environmental Stress. In Annual Fishes. Life History Strategy, Diversity, and Evolution, eds. N. Berois G. García and R. De Sá), pp. 159-184. Boca Raton, FL USA: CRC Press, Taylor \& Francis.

Podrabsky, J. and Wilson, N. (2016). Hypoxia and anoxia tolerance in the annual killifish Austrofundulus limnaeus. Integrative and Comparative Biology 56, 500-509.

Podrabsky, J. E. (1999). Husbandry of the annual killifish Austrofundulus limnaeus with special emphasis on the collection and rearing of embryos. Environmental Biology of Fishes 54, 421-431.

Podrabsky, J. E., Carpenter, J. F. and Hand, S. C. (2001). Survival of water stress in annual fish embryos: dehydration avoidance and egg envelope amyloid fibers. American Journal of Physiology 280, R123-R131.

Podrabsky, J. E., Garrett, I. D. F. and Kohl, Z. F. (2010a). Alternative developmental pathways associated with diapause regulated by temperature and maternal influences in embryos of the annual killifish Austrofundulus limnaeus. Journal of Experimental Biology 213, 3280-3288.

Podrabsky, J. E. and Hand, S. C. (1999). The bioenergetics of embryonic diapause in an annual killifish, Austrofundulus limnaeus. Journal of Experimental Biology 202, 2567-2580. 
Podrabsky, J. E. and Hand, S. C. (2000). Depression of protein synthesis during diapause in embryos of the annual killifish Austrofundulus limnaeus. Physiological and Biochemical Zoology 73, 799-808.

Podrabsky, J. E., Hrbek, T. and Hand, S. C. (1998). Physical and chemical characteristics of ephemeral pond habitats in the Maracaibo basin and Llanos region of Venezuela. Hydrobiologia 362, 67-78.

Podrabsky, J. E., Lopez, J. P., Fan, T. W. M., Higashi, R. and Somero, G. N. (2007). Extreme anoxia tolerance in embryos of the annual killifish Austrofundulus limnaeus: Insights from a metabolomics analysis. Journal of Experimental Biology 210, 2253-2266.

Podrabsky, J. E., Menze, M. A. and Hand, S. C. (2012a). Rapid Communication: Long-term survival of anoxia despite rapid ATP decline in embryos of the annual killifish Austrofundulus limnaeus. Journal of Experimental Zoology A: Ecological Genetics and Physiology 317, 524-532.

Podrabsky, J. E., Riggs, C. L. and Duerr, J. M. (2012b). Anoxia Tolerance During Vertebrate Development - Insights from Studies on the Annual Killifish Austrofundulus limnaeus. In Anoxia, (ed. P. Padilla), pp. 3-24. Rijeka, Croatia: InTech.

Podrabsky, J. E., Tingaud-Sequeira, A. and Cerdà, J. (2010b). Metabolic dormancy and responses to environmental desiccation in fish embryos. In Dormancy and resistance in harsh environments, eds. E. Lubzens J. Cerda and M. Clark), pp. 203-226: Springer.

Polačik, M. and Podrabsky, J. E. (2015). Temporary Environments. In Extremophile Fishes: Ecology, Evolution, and Physiology of Teleosts in Extreme Environments, eds. R. Riesch M. Tobler and M. Plath), pp. 217-245. Cham, Switzerland: Springer.

Polačik, M., Vrtílek, M., Reichard, M., Blazek, R. and Podrabsky, J. (2020). Embryo ecology: A critical role of environment in the diapause of wild annual fish populations. Freshwater Biology submitted.

Prentice, H. M. (2009). The major contribution of brain GABAergic function to anoxic survival. Physiological Genomics 36, 59-60.

Pri-Tal, B. M., Blue, S., Pau, F. K. Y. and Podrabsky, J. E. (2011). Hormonal components of altered developmental pathways in the annual killifish, 
Austrofundulus limnaeus. General and Comparative Endocrinology 174, 166174.

Qume, M. and Fowler, L. (1997). Effect of chronic treatment with the GABA transaminase inhibitors $y$-vinyl GABA and ethanolamine O-sulphate on the in vitro GABA release from rat hippocampus. British Journal of Pharmacology $122,539-545$.

Ricci, G., Vesci, L., Nardini, M., Arduini, A., Storto, S., Rosato, N. and Cavallini, D. (1989). Detection of 2H-1, 4-thiazine-5, 6-dihydro-3, 5-dicarboxylic acid (lanthionine ketimine) in the bovine brain by a fluorometric assay. Biochimica et Biophysica Acta (BBA)-General Subjects 990, 211-215.

Riggs, C. and Podrabsky, J. (2017). Small noncoding RNA expression during extreme anoxia tolerance of annual killifish (Austrofundulus limnaeus) embryos. Physiological Genomics 49, 505-518.

Riggs, C., Summers, A., Warren, D., Nilsson, G., Lefevre, S., Dowd, W., Milton, S. and Podrabsky, J. (2018). Small non-coding RNA expression and extreme vertebrate anoxia tolerance. Frontiers in Genetics 9, 230.

Romney, A., Davis, E., Corona, M., Wagner, J. and Podrabsky, J. (2018). Temperature dependent vitamin $\mathrm{D}$ signaling regulates developmental trajectory associated with diapause in an annual killifish. Proceedings of the National Academy of Sciences of the United States of America 115, 12763-12768.

Romney, A. and Podrabsky, J. (2017). Transcriptomic analysis of maternally provisioned cues for phenotypic plasticity in the annual killifish, Austrofundulus limnaeus. EvoDevo 8, 6.

Ruffmann, R., Schlick, R., Chirigos, M., Budzynsky, W. and Varesio, L. (1987). Antiproliferative activity of picolinic acid due to macrophage activation. Drugs under experimental and clinical research 13, 607-614.

Russell, J. J., Theriot, J. A., Sood, P., Marshall, W. F., Landweber, L. F., Fritz-Laylin, L., Polka, J. K., Oliferenko, S., Gerbich, T. and Gladfelter, A. (2017). Non-model model organisms. BMC Biology 15, 55.

Rust, C., Karnitz, L. M., Paya, C. V., Moscat, J., Simari, R. D. and Gores, G. J. (2000). The bile acid taurochenodeoxycholate activates a phosphatidylinositol 3-kinase-dependent survival signaling cascade. Journal of Biological Chemistry 275, 20210-20216. 
Saikumar, P., Dong, Z., Weinberg, J. M. and Venkatachalam, M. A. (1998). Mechanisms of cell death in hypoxia/reoxygenation injury. Oncogene 17, 3341-3349.

Saks, V. and Strumia, E. (1993). Phosphocreatine: molecular and cellular aspects of the mechanism of cardioprotective action. Current therapeutic research 53, 565-598.

Schieber, M. and Chandel, N. S. (2014). ROS function in redox signaling and oxidative stress. Current Biology 24, R453-R462.

Schoots, A. F. M., Stikkelbroeck, J. J. M., Bekhuis, J. F. and Denuce, J. M. (1982). Hatching in teleostean fishes: fine structural changes in the egg envelope during enzymatic breakdown in vivo and in vitro. Journal of Ultrastructure Research 80, 185-196.

Schwartz-Bloom, R. D. and Sah, R. (2001). y-Aminobutyric acid neurotransmission and cerebral ischemia. Journal of Neurochemistry 77, 353371.

Sidell, D. B. (1998). Intracellular oxygen diffusion: the roles of myoglobin and lipid at cold body temperature. Journal of Experimental Biology 201, 11181127.

Sinclair, B. J., Ferguson, L. V., Salehipour-shirazi, G. and MacMillan, H. A. (2013). Cross-tolerance and cross-talk in the cold: relating low temperatures to desiccation and immune stress in insects. Integrative and Comparative Biology $53,545-556$.

Sloley, B., Trudeau, V., Dantonio, M. and Peter, R. (1994). Persistent elevation of tissue GABA and serum gonadotropin concentrations by GABA transaminase inhibition in goldfish (Carassius auratus). Endocrine 2, 385-391.

Smith, R. W. and Houlihan, D. F. (1995). Protein synthesis and oxygen consumption in fish cells. Journal of Comparative Physiology B 165, 93-101.

Spustova, V. and Oravec, C. (1989). Antitumor effect of hippurate. An experimental study using various mouse tumor strains. Neoplasma 36, 317-320.

Steele, S. L., Chadwick, T. D. and Wright, P. A. (2001). Ammonia detoxification and localization of urea cycle enzyme activity in embryos of the 
rainbow trout (Oncorhynchus mykiss) in relation to early tolerance to high environmental ammonia levels. Journal of Experimental Biology 204, 2145-2154.

Sulkowski, P. L., Oeck, S., Dow, J., Economos, N. G., Mirfakhraie, L., Liu, Y., Noronha, K., Bao, X., Li, J., Shuch, B. M. et al. (2020). Oncometabolites suppress DNA repair by disrupting local chromatin signalling. Nature 10.1038/s41586-020-2363-0.

Tarasenko, T. N., Cusmano-Ozog, K. and McGuire, P. J. (2018). Tissue acylcarnitine status in a mouse model of mitochondrial $\beta$-oxidation deficiency during metabolic decompensation due to influenza virus infection. Molecular genetics and metabolism 125, 144-152.

Tauffenberger, A., Fiumelli, H., Almustafa, S. and Magistretti, P. J. (2019). Lactate and pyruvate promote oxidative stress resistance through hormetic ROS signaling. Cell death \& disease 10, 1-16.

Taylor, M. H. (1999). A suite of adaptations for intertidal spawning. American Zoologist 39, 313-320.

Ten Busch, M., Milakofsky, L., Hare, T., Nibbio, B. and Epple, A. (1997). Regulation of substances in allantoic and amniotic fluid of the chicken embryo. Comparative Biochemistry and Physiology Part A 116, 131-136.

Thompson, A. W., Hayes, A., Podrabsky, J. E. and Ortí, G. (2017). Gene expression during delayed hatching in fish-out-of-water. Ecological Genetics and Genomics 3, 52-59.

Thompson, J. W., Nilsson, G. E. and Perez-Pinzon, M. A. (2013). Anoxia Resistance in Lower and Higher Vertebrates. In Innate Tolerance in the CNS, pp. 19-35: Springer.

Tingaud-Sequeira, A., Zapater, C., Chauvigné, F., Otero, D. and Cerdà, J. (2009). Adaptive plasticity of killifish (Fundulus heteroclitus) embryos: dehydration-stimulated development and differential aquaporin-3 expression. American Journal of Physiology - Regulatory, Integrative and Comparative Physiology 296, R1041-R1052.

Tokarska-Schlattner, M., Epand, R. F., Meiler, F., Zandomeneghi, G., Neumann, D., Widmer, H. R., Meier, B. H., Epand, R. M., Saks, V. and Wallimann, T. (2012). Phosphocreatine interacts with phospholipids, affects membrane properties and exerts membrane-protective effects. PLOS ONE 7. 
Toledo, R. C. and Jared, C. (1993). Cutaneous adaptations to water balance in amphibians. Comparative Biochemistry and Physiology 105A, 593608.

Tsukatani, T., Higuchi, T. and Matsumoto, K. (2005). Enzyme-based microtiter plate assay for $\mathrm{\gamma}$-aminobutyric acid: application to the screening of $\mathrm{\gamma}$ aminobutyric acid-producing lactic acid bacteria. Analytica Chimica Acta 540, 293-297.

Vigorito, C., Anishchenko, E., Mele, L., Capolongo, G., Trepiccione, F., Zacchia, M., Lombari, P., Capasso, R., Ingrosso, D. and Perna, A. F. (2019). Uremic toxin lanthionine interferes with the transsulfuration pathway, angiogenetic signaling and increases intracellular calcium. International Journal of Molecular Sciences 20, 2269.

Wagner, J., Singh, P., Romney, A., Riggs, C., Minx, P., Woll, S., Roush, J., Warren, W., Brunet, A. and Podrabsky, J. (2018). The genome of Austrofundulus limnaeus offers insights into extreme vertebrate stress tolerance and embryonic development. BMC Genomics 19, 155.

Wagner, J. T., Knapp, M. J. and Podrabsky, J. E. (2019). Antioxidant capacity and anoxia tolerance in Austrofundulus limnaeus embryos. Journal of Experimental Biology 222, jeb204347.

Ward, P. S., Patel, J., Wise, D. R., Abdel-Wahab, O., Bennett, B. D., Coller, H. A., Cross, J. R., Fantin, V. R., Hedvat, C. V. and Perl, A. E. (2010). The common feature of leukemia-associated IDH1 and IDH2 mutations is a neomorphic enzyme activity converting a-ketoglutarate to 2-hydroxyglutarate. Cancer Cell 17, 225-234.

Wells, M. W., Turko, A. J. and Wright, P. A. (2015). Fish embryos on land: terrestrial embryo deposition lowers oxygen uptake without altering growth or survival in the amphibious fish Kryptolebias marmoratus. Journal of Experimental Biology 218, 3249-3256.

Williams, M., Wallace, S., Tyler, J., McCall, C., Gutierrez, A. and Spano, J. (1993). Biochemical characteristics of amniotic and allantoic fluid in late gestational mares. Theriogenology 40, 1251-1257.

Winston, P. W. and Bates, D. H. (1960). Saturated solutions for the control of humidity in biological research. Ecology 41, 232-237. 
Wolf, R. and Klemisch, H. (1991). Adaptation of an enzymatic fluorescence assay for L-glutamic acid decarboxylase. Analytical Biochemistry $192,78-81$.

Woll, S. and Podrabsky, J. (2017). Insulin-like growth factor signaling regulates developmental trajectory associated with diapause in embryos of the annual killifish Austrofundulus limnaeus. Journal of Experimental Biology 220, 2777-2786.

Wourms, J. P. (1972a). The developmental biology of annual fish II. Naturally occuring dispersion and reaggregation of blastomeres during the development of annual fish eggs. Journal of Experimental Zoology 182, 169-200.

Wourms, J. P. (1972b). The developmental biology of annual fishes I. Stages in the normal development of Austrofundulus myersi Dahl. Journal of Experimental Zoology 182, 143-168.

Wourms, J. P. (1972c). The developmental biology of annual fishes III. Pre-embryonic and embryonic diapause of variable duration in the eggs of annual fishes. Journal of Experimental Zoology 182, 389-414.

Wright, J. C. (1989). Desiccation tolerance and water-retentive mechanisms in tardigrades. Journal of Experimental Biology 142, 267-292.

Wright, P. A., Felskie, A. and Anderson, P. M. (1995). Induction of ornithine-urea cycle enzymes and nitrogen metabolism and excretion in rainbow trout (Oncorhynchus mykiss) during early life stages. Journal of Experimental Biology 198, 127-135.

Wu, Y., Wang, W. and Richerson, G. B. (2001). GABA transaminase inhibition induces spontaneous and enhances depolarization-evoked GABA efflux via reversal of the GABA transporter. Journal of Neuroscience 21, 26302639.

Xing, A., Li, X., Jiang, C., Chen, Y., Wu, S., Zhang, J. and An, L. (2019). As a Histone Deacetylase Inhibitor, $y$-Aminobutyric Acid Upregulates GluR2 Expression: An In Vitro and In Vivo Study. Molecular nutrition \& food research 63, 1900001.

Xu, W., Yang, H., Liu, Y., Yang, Y., Wang, P., Kim, S.-H., Ito, S., Yang, C., Wang, P. and Xiao, M.-T. (2011). Oncometabolite 2-hydroxyglutarate is a competitive inhibitor of a-ketoglutarate-dependent dioxygenases. Cancer Cell 19, 17-30. 
Zajic, D., Nicholson, J. and Podrabsky, J. (2020). No water, no problem: Stage-specific metabolic responses to dehydration stress in annual killifish embryos. Journal of Experimental Biology Submitted.

Zajic, D. and Podrabsky, J. (2020). GABA daba doo, anoxia got nothing on you: GABA metabolism is crucial for long-term survival in annual killifish embryos. Journal of Experimental Biology Submitted.

Zazueta, C., Buelna-Chontal, M., Macías-López, A., Román-Anguiano, N. G., González-Pacheco, H., Pavón, N., Springall, R., Aranda-Frausto, A., Bojalil, R. and Silva-Palacios, A. (2018). Cytidine-5'-diphosphocholine protects the liver from ischemia/reperfusion injury preserving mitochondrial function and reducing oxidative stress. Liver Transplantation 24, 1070-1083.

Zhang, D., Tang, Z., Huang, H., Zhou, G., Cui, C., Weng, Y., Liu, W., Kim, S., Lee, S. and Perez-Neut, M. (2019). Metabolic regulation of gene expression by histone lactylation. Nature $574,575-580$.

Zhang, Y., Vanmeert, M., Siekierska, A., Ny, A., John, J., Callewaert, G., Lescrinier, E., Dehaen, W., de Witte, P. A. and Kaminski, R. M. (2017). Inhibition of glutamate decarboxylase (GAD) by ethyl ketopentenoate (EKP) induces treatment-resistant epileptic seizures in zebrafish. Scientific Reports 7, 1-13.

Zweifler, R. M. (2002). Membrane stabilizer: citicoline. Current medical research and opinion 18, s14-s17. 
Appendix: Supplemental File Information

\begin{tabular}{|c|c|c|c|c|}
\hline File & Name & File size & File type & Software \\
\hline $\begin{array}{c}\text { Supplemental } \\
\text { Table S4.1 }\end{array}$ & $\begin{array}{c}\text { All metabolites identified in } \\
\text { embryos of Austrofundulus } \\
\text { limnaeus exposed to } \\
\text { dehydration stress due to } \\
\text { aerial incubation. }\end{array}$ & $344 \mathrm{~KB}$ & xlsx & $\begin{array}{c}\text { Microsoft } \\
\text { Excel }\end{array}$ \\
\hline
\end{tabular}

